\title{
WHAT DO THE EXPERIENCES OF JAPAN AND SWEDEN HAVE TO TELL US ABOUT SPECIAL EDUCATION POLICY AND PRACTICE IN THE UNITED STATES?
}

\author{
A Dissertation \\ Presented to \\ The Faculty of the Curry School of Education \\ University of Virginia
}

In Partial Fulfillment

of the Requirements for the Degree

Doctor of Philosophy

by

Charlene S. Pritzker, B.A., M.A., M.Ed.

December 2015 
(C) Copyright by Charlene S. Pritzker All Rights Reserved December 2015 


\section{ABSTRACT}

By a variety of measures, Sweden and Japan have both created educational systems that serve the great majority of their students, including many with disabilities, and consistently prepare them for full membership in their social, economic, and political communities. This dissertation examines the culture, policy, political, and legal factors relating to educational practice that appear to have affected their ability to achieve these results, and suggests ways that the United States might learn from their experience.

The research is a comparative case study of special education in Sweden and Japan, using elements of historical analysis to examine development and changes in special education since the 1970's. The study is based on an exhaustive literature search, including historical and government documents, research articles, commentaries and opinion pieces regarding special education in the two countries, focusing on both current educational policy and the forces over the past 40 years that led to these policies.

The study identified several key themes of difference between special education programs in Sweden and/or Japan and the United States, which can potentially inform practices in the United States. These include: (1) Providing all students (other than a very small number with low incidence-high impact disabilities) with access to the full, rigorous curriculum and standards offered to all students, thereby equalizing the basic inputs of education, and making it possible for all students to reach their maximum potential. When and where needed, this could be supplemented with appropriate remediation and basic skills instruction. (2) Basing the decision to provide services on demonstrated needs rather than a requirement for formal diagnosis or an assessment of discrepancy between "ability" and performance, so that students can get help with fewer 
procedural formalities, more quickly and for short or longer periods of time. (3) Focusing on creating an educational environment where a range of student needs can be accommodated and where the environment itself ameliorates the effects of disability so that fewer students will need to be identified and offered special services. (4) Extending the concept of inclusion to "normalization" in all aspects of life, as well as in the schools, providing more ways for individuals with disabilities to participate fully in the economy and civic and social life, both during school years and as adults. (5) Limiting the impact of administrative and legal burdens and costs on the practice and delivery of special education, freeing funds for direct educational needs. 


\section{DEDICATION}

This dissertation is dedicated to my family: my husband David, and our three children, Suzanne, Steven and Erica. Dedications to family are fairly typical, but it is probably rare to have a situation in which, among one's prime supporters, cheerleaders and role models are a Ph.D. student's grown children. Suzanne, our oldest child, after completing her bachelor's degree at the University of Virginia, continued at the University's Curry School to get a master's degree in Social Foundations, and then earned a Ph.D. in Social Work policy from Washington University in St. Louis. It was from talking with her about the Curry program that I got the idea to take courses in the Social Foundations Department to focus on the "big ideas" of education. When the going got tough, and I felt discouraged, Suzanne was able to mentor me and talk about her experiences as a doctoral student and as an advisor to doctoral students as a professor in the Graduate School of Social Work at the University of Houston. Steven and Erica, as well, were ongoing sources of encouragement, making clear that they were behind me and wanted me to take care of the "unfinished business" of completing my dissertation and earning the Ph.D. As for my husband, in addition to encouragement and unwavering faith that I could complete my work, he is the world's most detail-oriented editor, and without doubt, he has read my many drafts and overly ambitious literature summaries more times than anyone else. For all these things, I dedicate this work to all of them. 


\section{ACKNOWLEDGMENTS}

I want to acknowledge three groups of people who have supported me throughout my rather long journey toward completing this dissertation. The first, of course, is my very patient committee, Professors Diane Hoffman, Eric Bredo, Daniel Driscoll and Derrick Alridge, all of whom stood behind me, encouraged me, and, at the same time, held me to high standards throughout. I particularly want to thank Daniel Driscoll, who met with me many times at University of Virginia's Northern Virginia Center, and helped me sort through the mountain of research I had collected and cull what was crucial. I also appreciate Derrick Alridge's willingness to step in late in the process, and work with me to refine my topic to align more closely with issues of special importance to me. Diane Hoffman, my Committee Chair, practiced one of the educational principles I advocate in this dissertation, being willing to be flexible and encourage perseverance, while having faith that I could write a dissertation that meets the appropriately rigorous standards for a University of Virginia Ph.D. Finally, among this group, I want to acknowledge an additional early mentor, Professor Jennings Wagoner who, unfortunately, died before I completed this dissertation. When I took his History of Education class and wrote my personal "educational history," in which I noted that not having completed a Ph.D. was one of the unfinished pieces of my life, he strongly encouraged me to continue and finish my work.

The second group includes my colleagues at the small, creative, progressive independent school where I have taught for the past 25 years, Burgundy Farm Country Day School, in Alexandria, Virginia. After taking several courses at the Northern Virginia Center, I was able to begin the serious journey toward the doctoral degree because Burgundy was willing to support me during a sabbatical year, which I spent in residence at UVA, and during which I completed my course work and wrote my master's thesis. Burgundy also supported a three-week leave for me to go to Japan under the Japan Fulbright Memorial Fund Teacher Program, which inspired the Japanese part of my doctoral study. And, of course, like my family and my University of Virginia advisors, my colleagues encouraged me to continue and finish my work. So I owe a lot of this to Burgundy's support, both financial and personal.

Last, and in many ways, most important, are the many students I have taught over the years, whose mode or pace of learning was not typical. My experience with these students caused me to consider the questions raised in this dissertation. For what my students have taught me, I say, "Thank you!" 


\section{Table of Contents}

DEDICATION

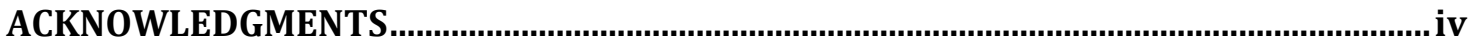

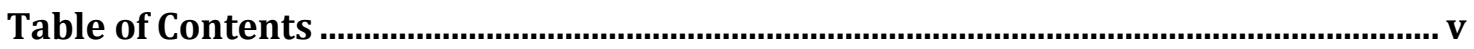

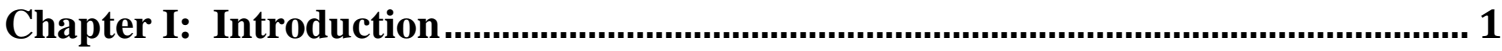

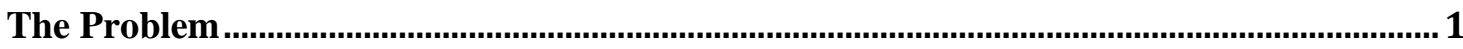

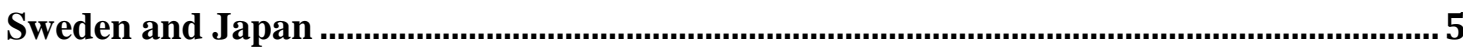

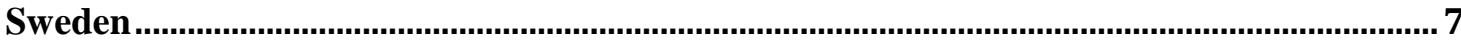

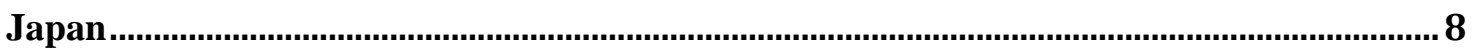

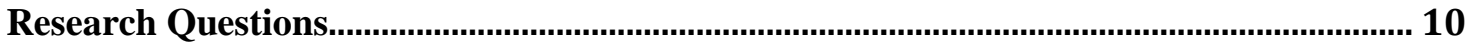

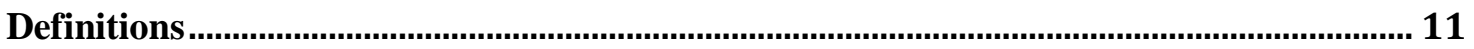

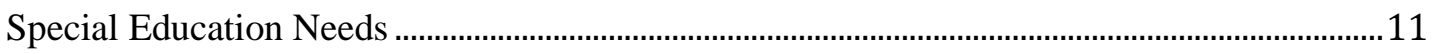

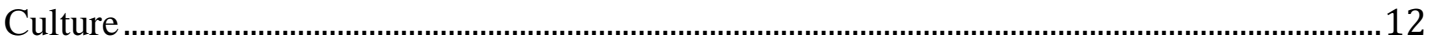

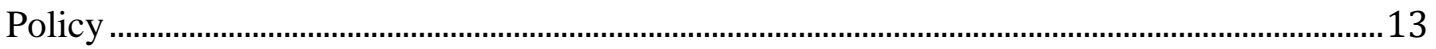

Significance of the Study: Implications for American Education..................................... 14

Dissertation Scope and Organization .................................................................................. 14

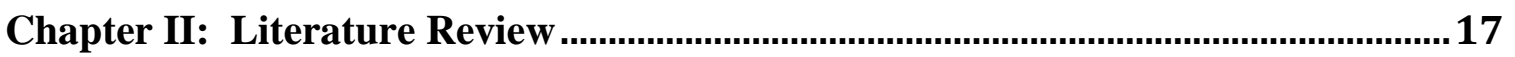

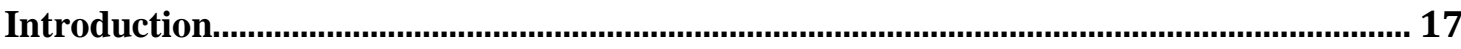

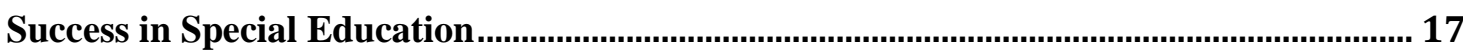

Problems in Special Education in the United States.................................................................. 20

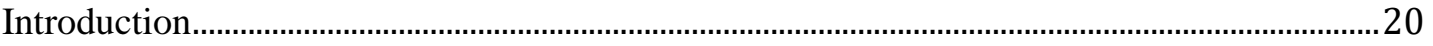

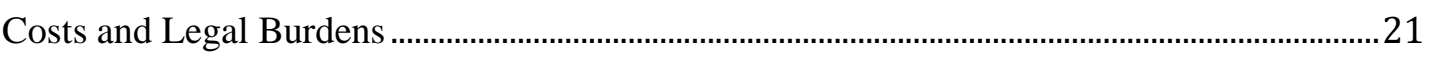

High School Graduation Rates ................................................................................................22

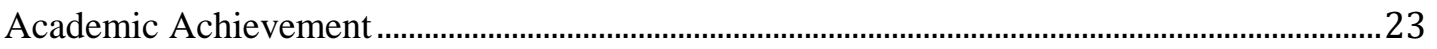

Preparation for Jobs, Independence and Continuing Education....................................................25

Fairness: Overrepresentation of Some Groups in Special Education...........................................26

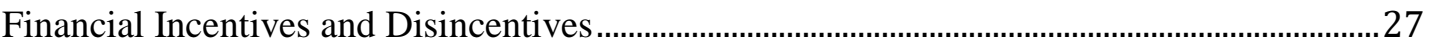

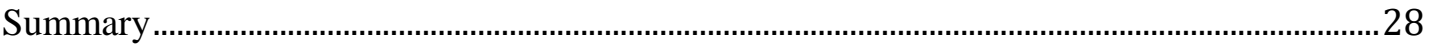

Commentary on Swedish Success and Challenges in Special Education ............................ 29

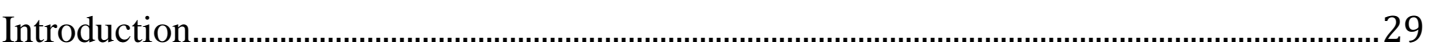

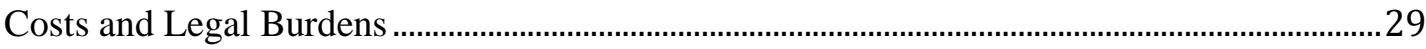

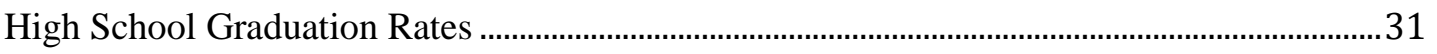

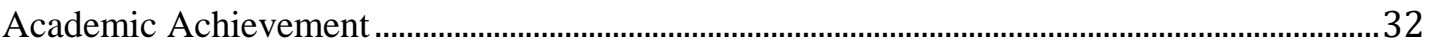

Preparation for Jobs, Independence and Continuing Education.....................................................33

Fairness: Overrepresentation of Some Groups in Special Education..........................................36

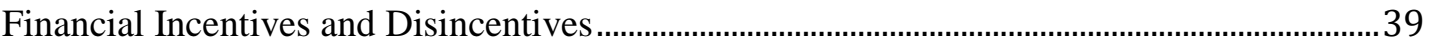

Commentary on Japanese Success and Challenges in Special Education........................... 41

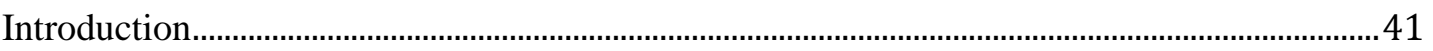

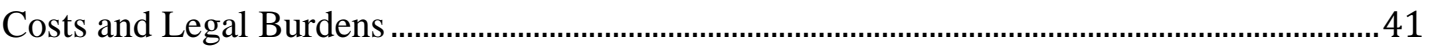

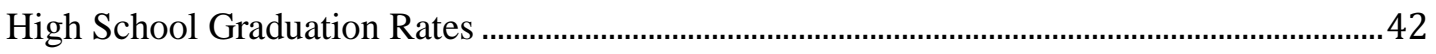




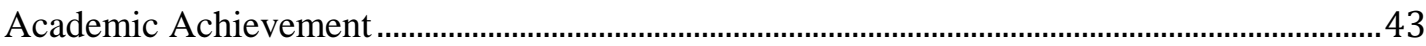

Preparation for Jobs, Independence and Continuing Education......................................................44

Fairness: Overrepresentation of Some Groups in Special Education............................................46

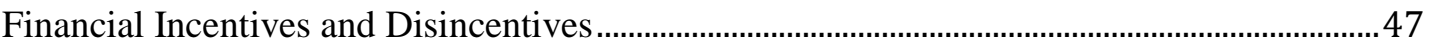

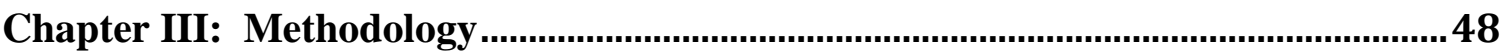

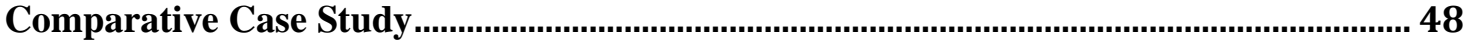

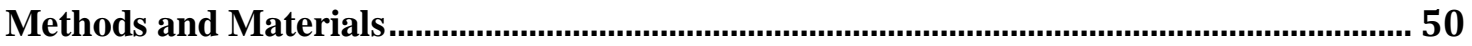

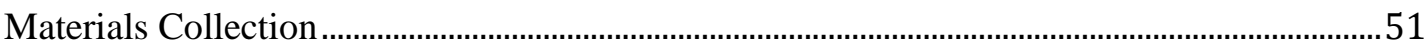

Materials Management and Analysis................................................................................... 52

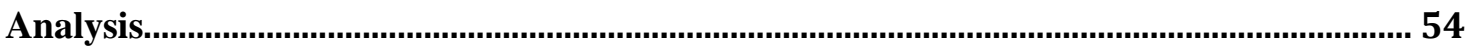

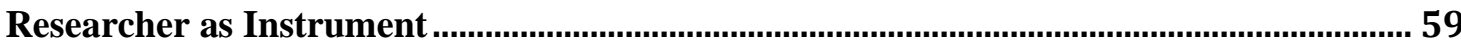

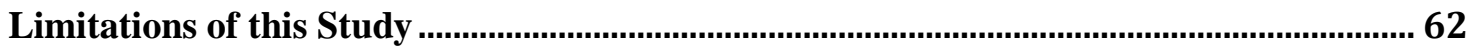

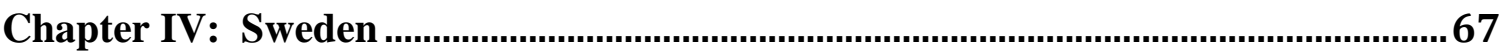

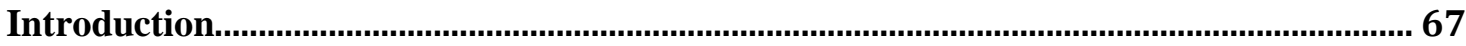

Part I Summary of the General Education System in Sweden ....................................... 69

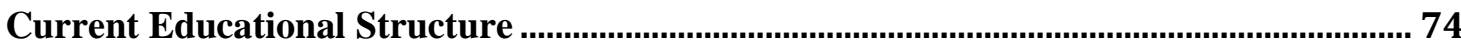

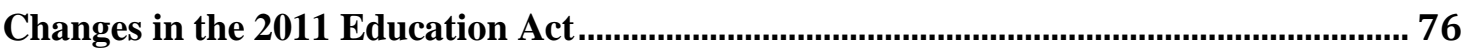

Part II Special Education in Sweden................................................................................ 77

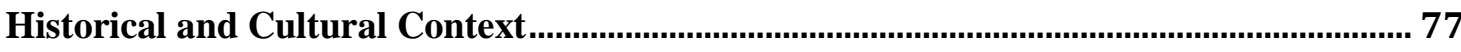

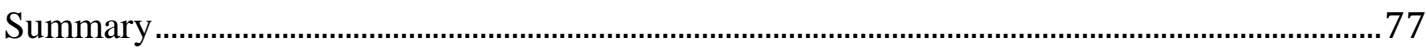

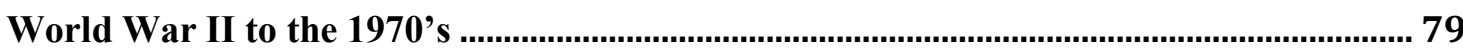

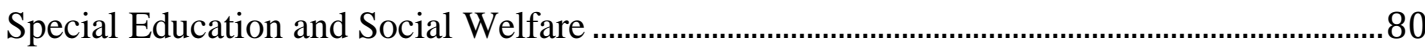

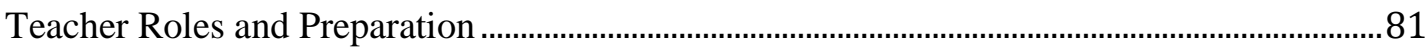

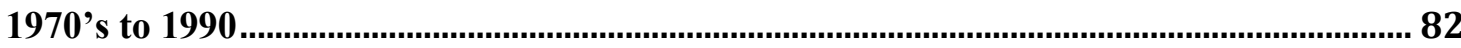

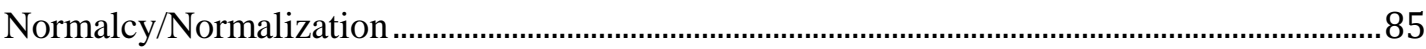

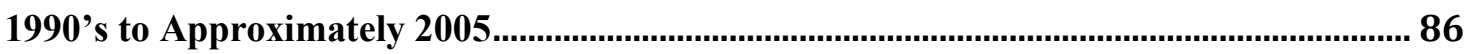

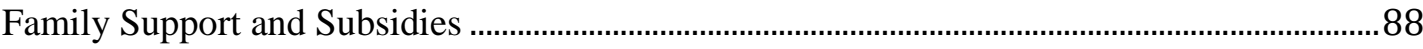

The Changing Role of Students and Teachers .......................................................................

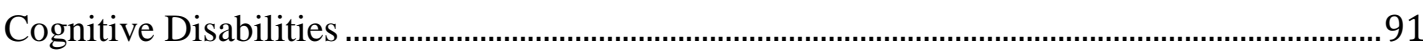

Upper Secondary, Tertiary and Adult Education.............................................................................92

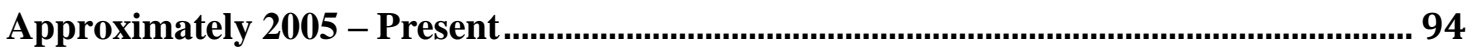

Performance at End of Compulsory School/Longitudinal Studies of Performance...................94

Reading/Dyslexic Difficulties................................................................................................ 95

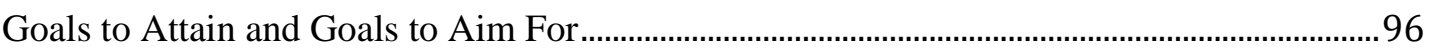

Education of Deaf/Hearing Impaired Students ..........................................................................97

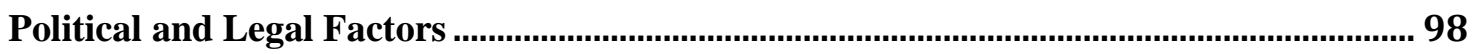

Critical Appraisals of the Changes in Swedish Special Education ............................................98

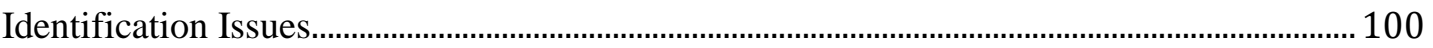

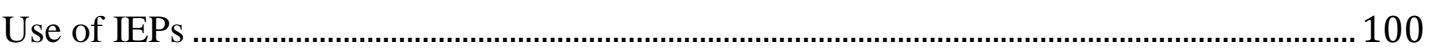

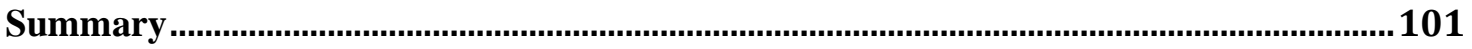

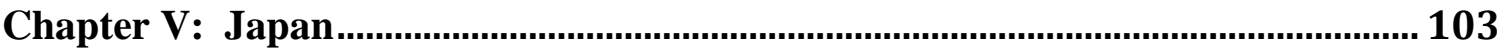

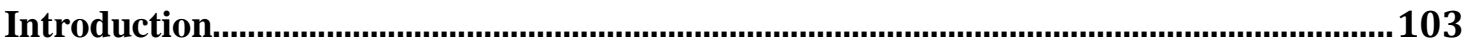


Part I Summary of the General Education System in Japan ............................................

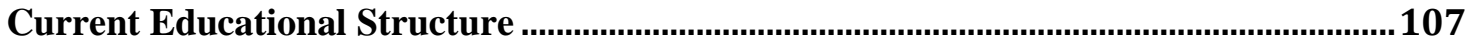

Current Issues—Recent and Proposed Reforms ...............................................................110

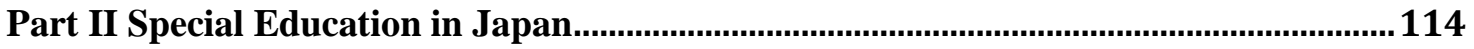

Historical and Cultural Context.................................................................................... 114

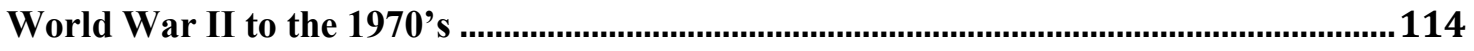

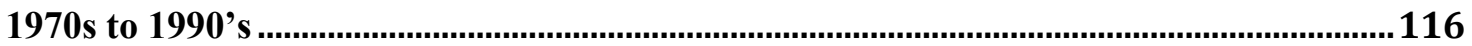

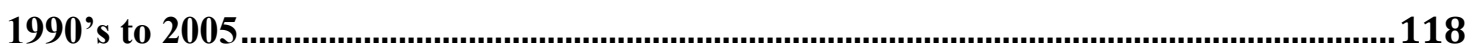

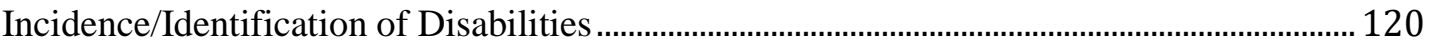

Teacher Training for Special Education ............................................................................... 122

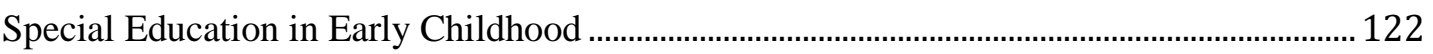

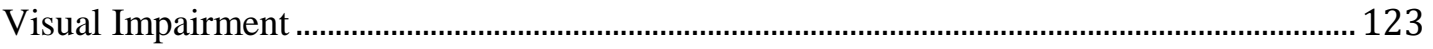

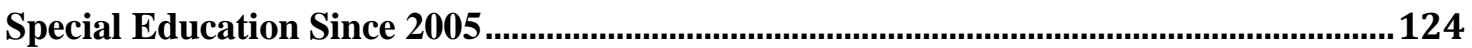

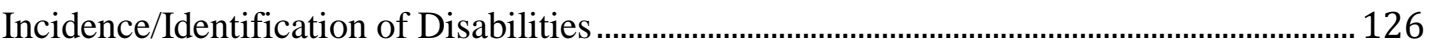

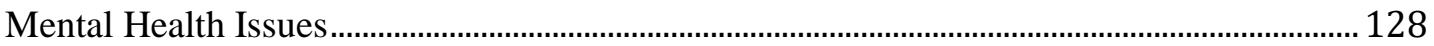

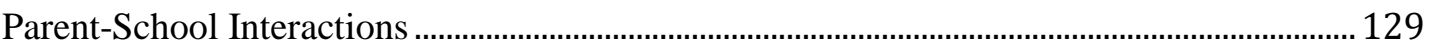

Mobility Problems and Lack of Facilities ................................................................................. 130

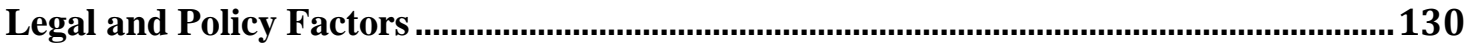

Laws and Government Policy Related to Special Education..................................................... 132

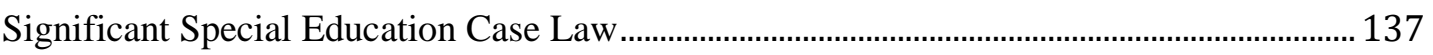

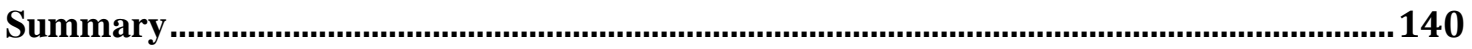

Chapter VI: Discussion, Analysis and Conclusions .............................................. 143

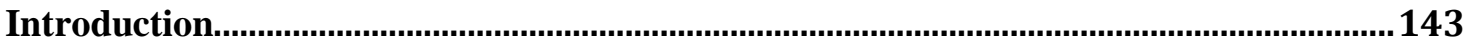

Cultural, Historical, Policy and Legal Factors Affecting Success in Swedish Special

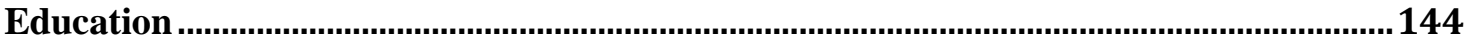

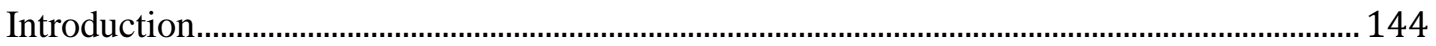

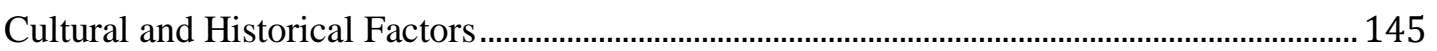

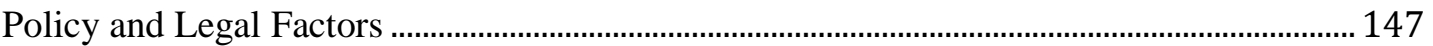

Cultural, Historical, Policy and Legal Factors Affecting Success in Japanese Special

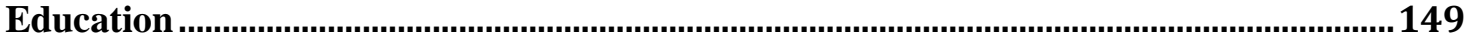

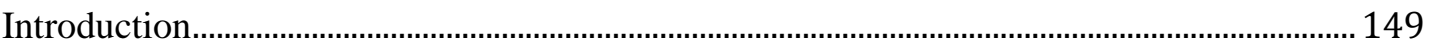

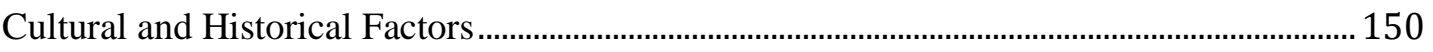

Policy and Legal Factors ...................................................................................................... 153

Common Themes and Effects on Success and Challenges in Sweden and Japan ............156

The Use of Ability as a Metric of Comparison with Performance ............................................. 156

Focusing on "Educational Needs" Rather than a Categorical Approach .................................. 158

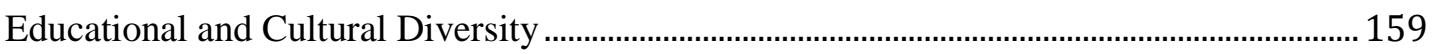

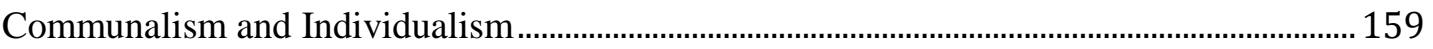

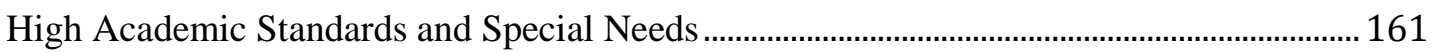

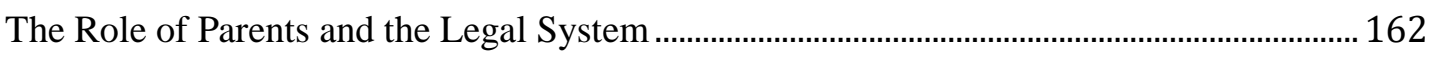

Fairness - Inputs and Outputs of Education .............................................................................. 163

Ameliorating Deficits and Full Access to a Common Curriculum ........................................... 164

Progressivism and Special Education .......................................................................................... 165 
Human Rights, Social Justice and Special Education Policies................................................. 165

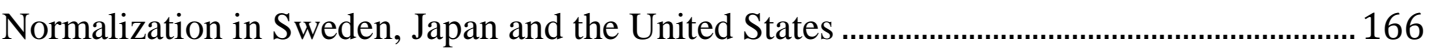

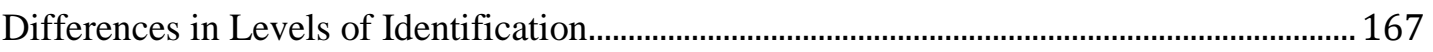

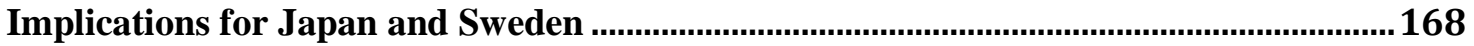

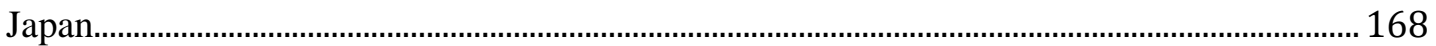

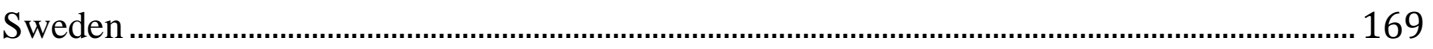

What We Can Learn: Implications for United States Special Education..........................171

Access to the Full, Enriched Curriculum for All Students, Balanced with Appropriate

Remediation and Basic Skills Instruction ......................................................................... 171

Perceived Needs vs. Formal Diagnosis....................................................................................... 173

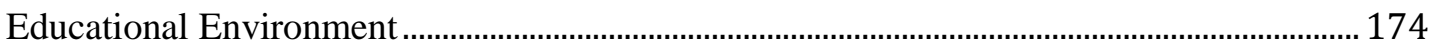

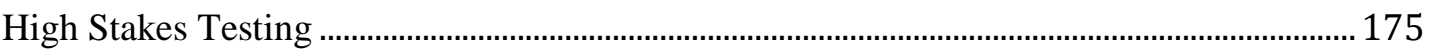

Flexibility in Time; Second and Third Chances; Adult Education .......................................... 176

Decreasing Administrative/Legal Burden of Special Education................................................ 177

Limitations Revisited: Effect on this Study's Conclusions ...........................................178

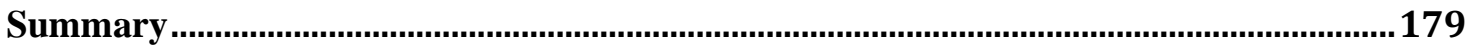

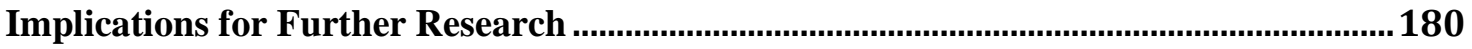

1. Extend Study to Another Strongly Individualistic Nation .................................................. 180

2. Extend to Other Non-Western Countries................................................................................... 181

3. Assess Benefit of Full Access to Curriculum vs. Remediation ............................................. 182

4. Focus More Closely on Elements Resulting in Acceptance or Rejection of Change...... 183

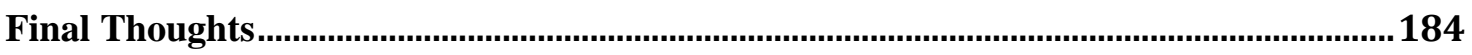

References ......................................................................................................................... 185 


\section{Chapter I: Introduction}

The way that special education is perceived and implemented varies significantly from one country and culture to another, and choices about how to educate students who learn differently or more slowly than is believed to be "normal" are made within the context of each country's social, political and legal environment. How a nation chooses to educate children who struggle in school reflects, among other things: how the culture defines such terms as ability, difference and diversity; whether it views the purpose of education as serving the general community or fulfilling individual needs; and how it views the interactions of its legal, social and educational systems. This dissertation examines the culture, educational policy, and political and legal structures in Sweden and Japan, which appear to have affected the ability of each nation to craft an educational system that serves the great majority of its students, including many with disabilities, and consistently prepares them for full membership in each nation's social, economic, and political communities.

\section{The Problem}

Currently, the United States identifies approximately 13\% (Aron \& Loprest, 2012) of all students as eligible for special education services. This does not include an additional at-least-one-percent of students granted accommodations under Section 504 (of the Rehabilitation Act of 1973, as amended) Plans (Holler \& Zirkel, 2008). The Individuals with Disabilities Education Act $^{1}$ (IDEA) mandates a system that identifies

\footnotetext{
${ }^{1}$ This legislation was first passed in 1975 as the Education for All Handicapped Law (PL 94-142), and then revised and amended as the Individuals with Disabilities Education Act (PL 108-446), and amended and revised since then. IDEA serves as the basis of American special education law.
} 
students as eligible for special education based on evidence of neurological dysfunction and measurable difference between "ability and achievement," and excludes difficulties that are deemed to be caused mainly by economic, social or linguistic differences or disadvantage. The United States system thus excludes from special education services those students who need help, short- or long-term, for any of a variety of reasons, including reasons of inadequate prior education or linguistic disadvantage, beyond completion of English as a Second Language courses, but cannot demonstrate either "dysfunction" or a measurable difference between scores on "ability" tests and tests of achievement. American special education and disability laws require that public schools provide a wide variety of services, as set forth in an Individualized Education Plan (IEP) for each identified student, and also provide for the possibility of a variety of educational accommodations under Section 504 of the Rehabilitation Act. In 2010, approximately 6.7 million students were served under IDEA. Of these, approximately $38 \%$ were served under the diagnosis of specific learning disability, and another $22 \%$ under speech or language impairment (Aron \& Loprest, 2012).

Special education services in the United States employ teachers, teacher aides, therapists, psychologists, counselors, supervisors, and other personnel, to provide education and mandated related services, and are estimated to cost approximately $90 \%$ more each year over and above the cost of educating students not identified as eligible for special education. Special education programs are funded by a combination of federal, state and local programs. In the 2009-2010 school year, federal funding was $\$ 12.5$ billion. The federal portion was originally intended to fund the excess cost of special education over general education, but has never fully or even substantially done so. In 2010, this 
funding was estimated to cover about $17 \%$ of the excess cost of special education. The remainder, a larger amount each year, is funded by states and localities (Aron \& Loprest, 2012; Chambers, Parrish, \& Harr, 2002). In 2012, there were approximately 3000 due process cases filed each year under IDEA, most by parents seeking to secure different or additional services for their children, under the FAPE (free and appropriate education) provision of IDEA, of which about $10 \%$ led to court cases (Nagano \& Weinberg, 2012). All of these cases and the legal apparatus of lawyers, courts and judges that support them come at a great cost to parents, to schools, and to the judicial system. In comparison, the number of court cases in Japan is in the single digits per year, and there are virtually none in Sweden.

In both the education of special education providers and in actual practice, American educators tend to focus on remediation of deficits. In their 2015 critique of special education, Danforth and Naraian (Danforth \& Naraian, 2015) point out the intellectual and practical knowledge base of special education has been the diagnosis and treatment of deficits. To the extent that this approach leads to students being separated from their peers, in different classes, different sections, or different reading groups, or "pulled out" to work with a learning specialist during class time, it may well deprive affected students of the intellectual challenge provided to all other students in the mainstream curriculum. There is little in special education textbooks or in most of the courses required for special education certification or offered to general education teachers that emphasizes making an active effort to include students receiving special education assistance in the more challenging discussions or in finding ways for them to take higher level classes. 
Despite the vast legal apparatus, the paperwork, time, and substantial cost of American special education programs, and the large number of children served, the United States continues to score relatively low among developed countries on international tests (Schleicher, 2014) and, more significantly, shows particularly large variance in scores compared to many of these other countries, indicating that a large number of children are not achieving an educational level needed for full participation in the American economy or for educated participation in the American democracy. On national tests, such as the National Assessment of Educational Progress (NAEP), it appears that there is a significant gap between the performance of general and special education students at all grade levels in both reading and mathematics. American students graduate from secondary school at a lower rate than do students in many other similar countries (approximately $75 \%$ of those students entering ninth grade, as compared to well over $90 \%$ in Sweden and Japan), and even though students served under IDEA graduate at a rate almost equal to general education students in the United States, a much smaller percent (46\% in 2005) receive a regular diploma (National Center for Education Statistics, 2015). Assuming that the distribution of disabilities is not very different among developed countries, this would indicate that Americans are not as successful at making it possible for students with disabilities to complete their education at a level that allows them the greatest possible chance of economic and personal success.

However, Sweden and Japan, which have two very different approaches to educating students who struggle in school or who learn differently, have both been very successful in educating the great majority of students, including, it appears, many of those students who would be identified as eligible for special education services in the 
United States. Since both countries formally identify only a very small percent of students as requiring special education, we must assume that many others, not identified in Sweden or Japan, would qualify for identification in the United States. By studying the special education systems (and the related general education systems) of Sweden and Japan, this dissertation looks at the educational factors that may lead to success in those countries with students who learn differently or more slowly than is the norm. While there are certainly other, non-educational factors, such as lower income disparities, ${ }^{2}$ relatively homogeneous societies, ${ }^{3}$ universal health care, etc. that may come into play and may explain a part of these differences, this dissertation focuses on the educational factors that may explain why both Sweden and Japan appear to achieve greater success in educating all students, resulting in higher general performance and significantly lower variance.

\section{Sweden and Japan}

Sweden and Japan were chosen for this study because they represent, among developed countries, two very different approaches to special education policy and practice, as well as two very different cultural contexts. They also represent differing approaches to dealing with concepts of ability, disability, difference and diversity. At the same time, both countries have been notably successful, by international standards, in

2 The United Nations Development Programme (http://hdr.undp.org/en) publishes data on income inequality, such as the ratio of the average income of the richest $10 \%$ of the population to the poorest $10 \%$. That ratio is 4.5 for Japan, 6.9 for Sweden, and 15.9 for the United States.

3 See data from http://www.worldpopulationstatistics.com/japan-population-2013/ cited above. As a comparison, the comparable data for the United States, http://www.worldpopulationstatistics.com/us-population-2013/ is: The largest ethnic group in the United States of America is those that identify as white, which makes up about $72 \%$ of the population. The next largest ethnic population is the Black American group, which makes up about $13 \%$ of the population. The Asian population makes up about $5 \%$ of the population and is thought to be the fastest growing ethnicity in the entire country. The rest of the population is made up of Hispanics and Latinos, American Indians, Alaskan Natives, Native Hawaiian, Pacific Islanders, and those that identify as multiracial. 
educating the great majority of their students, and have low illiteracy and high secondary school graduation rates. In addition, these countries were chosen for practical reasons. Much of the education literature published in or about Japan and Sweden is published in English. ${ }^{4}$ Furthermore, although it is now several years ago, the author of this study had the opportunity to spend three weeks in Japan during 2005, as a Japan Fulbright Memorial Fund Scholar, studying and observing Japanese education, and was able to meet with and interview teachers, administrators and education professors regarding Japanese approaches to special education. This experience gave her a much better understanding of Japanese educational practice and filled in some gaps that result from an inability to read Japanese.

Meijer (Meijer, 1998) described countries as having "one-track," "two-track" or "multi-track" forms of special education policy. One-track countries integrate almost all students into mainstream schools serving everyone. They generally label only a small percentage of students as having special education needs, and have less than $1 \%$ of students in special schools or classes. Two-track countries often have two separate laws for mainstream and special education, and operate quite separate systems to serve each of these streams. The special education system in these countries tends to be comparatively large and very differentiated, with many disability categories. These countries often have more than $3 \%$ of students in special education settings separate from the mainstream, but label very few students within mainstream education as having special education needs. Multi-track countries generally have a continuum of services between the single mainstream approach and the single alternative offered in the two-track system. Available

\footnotetext{
${ }^{4}$ As noted in the limitations section in Chapter III, there are significantly fewer articles available in English about Japan than about Sweden.
} 
services include a variety of part- or full-time special classes, and special arrangements within both mainstream and special schools. These countries tend to have 1-3\% of students in special settings, but tend to identify high numbers of students as having special education needs.

Sweden falls into the one-track category, with a small number of identified students, and almost all students integrated into a "school for all." Japan, in terms of the number of students it identifies and the small number in special schools or classes, might be thought of as falling into the one-track category, but it is essentially a two-track system because Japan has historically, and until fairly recently, provided a fully separate special education system, with special schools or classes, organized by category, and for all other students, virtually no special support for children who struggled in regular classrooms. In contrast, the United States falls within the multi-track description, identifying a far larger percent of students than either Japan or Sweden, and offering a continuum of services.

\section{Sweden}

Sweden's approach to special education is a clear example of Meijer's (Meijer, 1998) one-track system. A relatively small percentage of students, in the $1-2 \%$ range, are formally identified as special education students, and a relatively large fraction of those so identified are served in separate schools or separate classes within mainstream schools. Swedish sources emphasize the concept of a "school for all," in which all students, other than the small number of identified special education students, receive the same nationwide curriculum and are expected to meet the same standards. Yet Sweden achieves almost universal literacy within the public compulsory schools, with relatively modest variance in performance. The Swedish education system fully acknowledges and 
embraces the vast differences in how, and how fast, different students learn, and provides assistance and accommodations, all supported by government funds, to those students having difficulty, literally from the moment of birth through both higher education and a substantial adult education program.

Sweden's success is accomplished through its long-standing political focus on social liberalism, social equity and community, which includes family and economic support for individuals with disabilities and its long-standing focus on "normalization" in living conditions, occupational and educational opportunities for all, even those with very serious disabilities. It also has a commitment to providing assistance to all students, for as long or short a period as needed, mostly without the burden of official identification and, despite fairly strict nationwide standards for each educational level, a flexibility that allows students to complete these standards on a timetable that enhances success. Looking at learning differences as diversity rather than deviance, however, leads to a comprehensive school setting in which educators attempt to make activities more inclusive by decreasing the barriers to exclusion. There has been virtually no litigation about issues related to special education.

\section{Japan}

A very small percentage of all Japanese students are identified as "special education students." At least for the compulsory schooling period, grades 1-9, Japan educates all non-special education students in heterogeneous classes, with little or no special support services, using the same nationally mandated curriculum for all. There is little or no special accommodation or assistance within the regular school day, other than individual teachers working with and helping students in their class, and virtually no specialists, speech or occupational therapists or counselors, to help students who have 
difficulty learning. Even after the passage of the Special Needs Education Law of 2007, which first officially recognized and defined the high incidence disabilities such as attention deficit disorder (ADD), learning disabilities (LD) and high functioning autism, Japanese schools have been very slow to adopt many of the ideas in the 2007 law, probably because they clash with basic cultural precepts.

At the same time, Japan's scores on a variety of international examinations indicate that Japanese students consistently perform at a very high average level, but also that there is a relatively low standard deviation in their scores, implying that few students were scoring far below (or above) the comparatively high level of their peers.

Several cultural and structural factors appear to contribute to Japan's success despite its apparently low expenditure of resources on special education. A cultural tradition that attributes differences in performance not to differences in ability but to the degree of effort and perseverance creates an environment in which students, teachers, and families commit themselves to high levels of effort when they meet educational challenges. Because grouping by ability violates the Japanese concept of the right to an equal education and full membership in the class community, all Japanese students in grades 1-9 receive the same full national curriculum, materials and instruction and are held to the same expectations.

Japanese culture has emphasized the importance of community. Individuals achieve their identity by their place in the communities to which they belong - their family, their class, their school, and later the company they work for. Japanese education is a very conscious transmitter of cultural values, and great attention is paid to the community building and behavioral aspects of education. For example, classrooms are 
structured in ways that appear to support the learning of students with some common learning issues.

On the other hand, the relatively small percent of students with significant, low incidence physical or cognitive disabilities are still assigned to separate schools or classes, with little decision-making inputs by parents, and with no presumption of inclusion or placement in a least restrictive environment, and with no expectation of "normalization." In fact, students, once diagnosed with a disability, have to demonstrate that they can function in a regular school environment in order to be awarded an "exception" from their placement in a special school. Because there is no human rights language in Japanese education law, and because Japanese culture tends to deal with difficult situations by finding ways to avoid confrontation, there has been almost no litigation about special education in Japan, and thus there are very few legal cases which challenge the presumption of separate education or assert the rights of children to receive services.

\section{Research Questions}

The broad questions that this study poses are what can American educators learn from the way two developed countries, Sweden and Japan, approach special education policy and practice, and how might these policies inform change and improvement in American education. In responding to these broad questions, this study specifically answers the following questions:

I. What policies, initiatives, and educational practices in Sweden and Japan lead to successful education of students in those countries?

II. What are the cultural and historical factors that enable Sweden and Japan to experience success with the great majority of students? 
III. What are the policy and political factors that enable Sweden and Japan to achieve success with the great majority of students?

IV. What legal factors and approaches enable Sweden and Japan to achieve success with the great majority of students?

V. Why is special education more "successful" in Sweden and Japan than in the United States (as measured by metrics of success such as limiting costs and legal burdens to maximize the ratio of benefits to costs; high school graduation rates; academic achievement; preparation for jobs, independence and continuing education; fairness; and financial incentives and disincentives)?

The objectives of this study are to identify approaches to special education that have led to a degree of success in Sweden and Japan and which have the potential to enhance special education practice in the United States.

\section{Definitions}

Several specific terms are used throughout this study. Because these terms may be used differently by different authors and in different educational contexts and cultures, the following definitions clarify their usage in this dissertation.

\section{Special Education Needs}

Throughout this dissertation, the phrase, students with "special education(al) needs," refers to those students who have difficulty succeeding in school without additional help, beyond what is considered normal teaching and assistance in the classroom. The term "special education" refers to the extra or "different" educational assistance, materials or allowances they receive. However, any such choice of terminology incorporates a set of biases and limitations. Whether a given student can be described as having "special education needs" may depend on the nature of schooling, the 
demands and expectations placed on students by the school or by society, the particular environment of the school, and what help is normally available in the classroom. The concept of "need" itself is dependent on the cultural context. For example, a student who has difficulty with arithmetic may not have "special education needs" if he or she is not required to take or pass a course in mathematics, or is permitted to use a calculator, or if his/her performance in mathematics is determined by the success of his/her working group, rather than individual skill. In a culture where conformity is prized and differences tend to be minimized, educational difficulty may have to rise to a more severe level to be considered an "educational need" that cannot be handled within the normal patterns of teaching and learning.

While normally it is students who are said to have educational "needs," it is sometimes the "needs" of teachers, parents or educational systems that determine whether a student is determined to have such a "need." Nevertheless, the term "special education needs" is used here because, among all terms currently used to describe students in discussions of special education policy, it appears to be the most neutral, implying nothing about the nature or cause of a student's difficulty or about the existence or nature of specific kinds of interventions. An understanding of what constitutes an educational "need" in each of the societies is thus a crucial part of this study.

\section{Culture}

Although there are varied ways of studying and characterizing "culture," it is generally defined by anthropologists as the pattern of artifacts, knowledge, skills, behaviors, attitudes and beliefs produced by a human society and transmitted from one 
generation to another (Reagan, 2005). ${ }^{5}$ Among the characteristics that can distinguish one culture from another are language, behavioral norms, religious and spiritual beliefs and practices, as well as political and social attitudes and values. Culture may be characterized as the distinctive activities a human community engages in, the methods and tools it chooses to carry out those activities, as well as the ways it thinks, values and uses language to talk about its activities.

There has been much dispute about the definition of "culture" and how best to study it, and approaches to the study of culture have changed substantially over the past 50 years. Sherry Ortner's seminal article (Ortner, 1984) traced changes in the way anthropologists approached the study of culture beginning in the 1960's, from an early focus on public symbols, through more structural, Marxist and political approaches.

\section{Policy}

Policies are explicit statements of governing principles formulated to regulate conduct within a political jurisdiction. Unlike statutes, they are often not enacted by a legislature, but rather through administrative action, although a legislative act may set broad guidelines or goals for implementation. Cultural factors affect the ways a society carries on many of its functions, including education. The widespread common beliefs and values that make up culture are, in essence, the assumptions that are accepted and considered "normal" by the majority of the members of that culture. They become part of specialized talk and ultimately incorporated into laws, regulations, and policies, often without being explicitly stated.

\footnotetext{
${ }^{5}$ Although this definition comes from Reagan's work, it is similar to definitions found in many other places.
} 


\section{Significance of the Study: Implications for American Education}

This study of special education policy and practice in Sweden and Japan offers the possibility for Americans to transcend the limitations resulting from a single cultural view of the world, and forces us to view American practices more objectively and critically, and with greater understanding of the elements of American culture, policy, politics and legal structure that underlie them. Because this study looks at how other countries have addressed the specific problem of educating students who may have difficulties with learning because of physical, mental or learning issues, problems that are common to the United States, Sweden and Japan, it enables us, as Americans, to take a bird's-eye view of the commonalities of educational issues surrounding special education, and of a wider selection of possible solutions than we might consider if we rely solely on our own cultural, political and educational resources. Thus, the results and conclusions of this study can help educators in the United States understand avenues for improving the success of American special education programs, leading to fewer students who are unprepared for the world of work or for full participation in America's economic, political and social life.

An additional significant value of this study is that its analysis and conclusions can help American educators gain an understanding of how other countries with different cultural perspectives have approached the concepts of "difference" and "disability," as an aid to examining American presuppositions and questioning the ideas our culture takes as starting points in constructing special education policy.

\section{Dissertation Scope and Organization}

This study examines the experiences of Sweden and Japan in dealing with students who may learn differently or may struggle with school and asks what their 
educational practices have to tell us about special education policy and practice in the United States and how we might improve it.

This study is organized in six chapters:

- Chapter I provides an introduction to the dissertation, and explains the problem investigated. It offers definitions of basic terms used in the study, the research questions this study addresses, a short discussion of the significance of the study, and a description of the scope and organization of the paper.

- Chapter II is a review of literature addressing what scholars have said about what constitutes success in special education, what problems have been observed in special education in the United States, how scholars have evaluated special education in Sweden and Japan, and how they explain their successes and challenges.

- Chapter III describes the methodologies used, and discusses limitations and potential biases that may have influenced the study.

- Chapters IV (Sweden) and V (Japan) each summarize the current state of general and special education in the country and trace the history of the development of current policy and practice, in order to shed light on the cultural, political and policy, legal, and economic issues that arose in each country and how these issues were addressed. In each of the country chapters, the material is presented chronologically to highlight and illuminate the changes that occurred in the nature of the discourse, as well as the evolution of topics and questions considered and discussed by scholars and educators at various levels. This kind of presentation allows a view of how the discourse has changed over the past 45 years in each country; of the language, issues and distinctions that were made (or not made); and how those distinctions worked to 
affect policy within each country.

- Chapter VI is a discussion and analysis that compares cultural, political and policy, and legal factors that affect and determine the nature of special education practice in Sweden and Japan, and that may be factors in the apparent success that these two countries have achieved in educating students with learning differences. It then offers implications for American special education based on the findings, and specific suggestions for Americans to consider as they continue to look at ways to increase the number of students who perform well and who are able to attain full and satisfying careers and social lives. Finally, it also suggests avenues for continued study. 


\section{Chapter II: Literature Review}

\section{Introduction}

This chapter has four sections. The first section reviews the literature that addresses how scholars have defined success in special education and lists several metrics that will be used throughout this study to assess the successes and challenges faced by special educators in Sweden, Japan, and the United States. The second section looks specifically at what the research says about problems and challenges in American special education (with the full understanding that the United States has also been successful in many ways, especially in extending guarantees for educational support to a large number of students with disabilities). The third and fourth sections examine how scholars have evaluated special education in Sweden and Japan, respectively, describing particular areas of success which merit study, as well as areas of continuing concern.

\section{Success in Special Education}

In order to compare and learn from approaches to special education in other countries, it is important to look at how success has been defined by scholars, policy makers, and other commentators on special education. As might be expected, success in special education has been defined in many different ways, using both quantitative and qualitative metrics. The commonly used quantitative ones, such as scores on internal (state and national) and external (international) examinations, graduation rates, levels of employment and economic self-sufficiency, etc. are, by their nature, the easiest to measure, but they are not the only markers of a successful special education program. 
Wehman, et al. (Wehman et al., 2015), for example, defined success in special education in terms of the percent of students who obtained competitive employment at any time during the first six years post-high school. How a researcher sets these time limits would affect the results and whether special education programs appear successful. Even defining high school graduation rates can be controversial. Legislation in the United States under the "No Child Left Behind Act" defined the graduation rate as the percent graduating four years after entering ninth grade, with some exceptions allowed for students with disabilities. Alternatively, using data from the National Longitudinal Transition Study-2 (NLTS2, 2010), Laura Schifter (Schifter, 2011) looked at high school graduation rates in the United States, and how long it took students with disabilities to graduate. She reported that $72.4 \%$ of students with disabilities had graduated within eight years after entering ninth grade, with a maximum conditional probability after five years. So success in special education might be defined in terms of 4-, 6-, or 8-year times posthigh school entrance, or in terms of graduation by the time students "age out" of stateprovided special education, at age 21 , and all of these variants appear in the literature. Further, Schifter noted that "high school graduation" is a somewhat nebulous term, because it may or may not include achievement of a General Education Development (GED) certificate instead of a traditional high school diploma, and it may or may not refer to receiving a lower-level diploma than a "regular" high school diploma. Since a substantial number of special education students achieve high school graduation, but not what their state considers a "regular diploma," this is a significant distinction.

Another approach to measuring "success" in special education is looking at scores on state-run examinations, such as the Virginia Standards of Learning tests or other states' 
Common Core examinations, on nationally-administered examinations, such as the National Assessment of Educational Progress (NAEP), or alternatively on international examinations, such as the Trends in International Mathematics and Science Study (TIMSS) or the Programme for International Student Assessment (PISA) (Provasnik, Gonzales, \& Miller, 2009), (Schleicher, 2014). However, results on international examinations may not be strictly comparable because different countries identify and exclude different number of students as "disabled" from the group that takes the tests. Whether or not states permit accommodations during testing, and the fact that allowable accommodations have changed over time, further make accurate comparisons difficult or unreliable.

Still another way to look at success in special education is to create some kind of cost-benefit analysis that takes into account both outcomes and the costs per student above the cost of education in a regular classroom. This metric of success would consider the high costs for special education in the United States, including the costs of legal actions, and measure these costs against the apparent achievements.

However, as John and Patricia Lloyd observed in their review of the accomplishments and challenges in special education during their 50-year careers (Lloyd \& Lloyd, 2015), successful special education cannot be measured only by these quantifiable metrics, but by whether and how many students in special education can take their full place in their country's society. They say:

Important outcomes are not just how many of our students participate in general education classes, but how many of them actually learn to read, to solve word problems, to discuss the causes of societies' problems, to cooperate with peers... Some metrics (e.g., graduation rates) may be helpful for policy purposes, but it is more important to know whether our 
students can succeed in work, can live on their own, can get along with others in their adult lives. (p. 80).

While these less easily measured characteristics of success are perhaps the most important, it appears that, for most students, a prerequisite would be attainment of the basic skills required for functioning in our complex society. That is, that attainment of basic skills, measured perhaps through test scores or graduation rates, is a necessary, although not sufficient condition for some of the more complex measures of success such as career attainments and personal satisfaction.

This study will look at all of these measures of success, although it is difficult to compare the more intangible methods suggested by the Lloyds. It should be noted, of course, that both Sweden and Japan officially identify only about one percent of their students as eligible for special education (although Sweden provides assistance in and out of the classroom for many more), making comparisons even more difficult.

The following sections summarize the principal literature on problems in American special education and the relative success of addressing the needs in Sweden and Japan. Each section is organized to address the following key issues: costs and legal burdens; high school graduation rates; academic achievement; preparation for jobs, independence and continuing education; fairness; and financial incentives and disincentives.

\section{Problems in Special Education in the United States}

\section{Introduction}

Special education in the United States has made vast improvements in the education of American children with disabilities over the 40 years since the passage of the Education for All Handicapped Children Act in 1975, a legal framework that 
subsequently became known as the Individuals with Disabilities Education Act (IDEA).

Some of these developments, particularly noted in the 10 years from 2000 to 2010 ,

include (U.S. Department of Education, 2010):

- Consistently increasing early interventions for children from birth through age two;

- Increasing numbers of students with disabilities attending neighborhood schools and educated for at least part of the day in general education classrooms;

- Increased rates of high school graduation, and fewer students dropping out of high school;

- More young people with disabilities attending both two- and four-year colleges; and

- More young adults no longer in school are employed (although not necessarily full-time).

Yet despite these clear improvements, it is clear that more progress is needed on

several of the success metrics, as well as on cost-benefit measures.

\section{Costs and Legal Burdens}

Among the characteristics of American special education of great concern is the

extraordinary level of expenditures the United States makes on special education services, estimated to be $90 \%$ above expenditures for general education students, ${ }^{6}$ not including any additional funds spent for students with Section $504^{7}$ accommodation plans. Aron

\footnotetext{
${ }^{6}$ To be sure, a great percentage of that is spent on the most severely disabled students and those in placements outside the public schools. But the amount, even for students educated in regular schools and in regular classrooms for all or part of the day is still well over $\$ 5000$. The $\$ 5000$ number was quoted for the 1999-2000 school year in (Chambers, Parrish, \& Harr, 2002).

${ }^{7}$ Section 504 of the Rehabilitation Act of 1973, as amended (Section 504). Section 504 is a federal law designed to protect the rights of individuals with disabilities in programs and activities that receive Federal financial assistance from the U.S. Department of Education (ED). Section 504 provides: "No otherwise qualified individual with a disability in the United States ... shall, solely by reason of her or his disability, be excluded from the participation in, be denied the benefits of, or be subjected to discrimination under any program or activity receiving Federal financial assistance ...." The Section 504 regulations require a school district to provide a "free appropriate public education" (FAPE) to each qualified student with a disability who is in the school district's jurisdiction, regardless of the nature or severity of the disability. Under Section 504 , FAPE consists of the provision of regular or special education and related aids and services designed to meet the student's individual educational needs as adequately as the needs of
} 
and Loprest note that there is little evidence about the effectiveness of many of the services provided to special education students, especially considering the expense and bureaucratic complexity of the system (Aron \& Loprest, 2012).

In addition, these expenses do not include the very substantial expenses in legal fees, court costs and the value of parents' and school officials' time in pursuing or responding to the large number of due process administrative cases and ultimately court cases that result. In his review of the rising number of education-related court cases, Lehigh Professor Perry Zirkel noted that federal special education cases showed a dramatic increase - from 623 decisions in the 1990s to 1,242 decisions between 20002010 (Samuels, 2011). These decisions are the tip of the iceberg, because only about one in ten due process hearings ultimately results in a court case. This means that in 2010, there were more than 10,000 due process hearings, most brought by parents who disagreed with the school special education committee on placement or extent of services for their children. All of these result in substantial costs, both financial and in terms of the time devoted by school officials, parents and court officials.

\section{High School Graduation Rates}

While high school graduation rates for both identified special education students and those who have no such identification are increasing, and graduation rates for special education students have approached those for students not so identified, both of these rates, on the order of $75 \%$ of those students entering ninth grade, are significantly below the corresponding figures for Sweden and Japan, which are well above 90\%. Also, far fewer special education students in the United States complete high school with a regular who are not covered under the specific requirements of IDEA. See: http://www2.ed.gov/about/offices/list/ocr/504faq.html. 
diploma, limiting their post-graduation options, and graduation rates depend significantly upon the kind of disability, with students who have sensory disabilities doing far better on this metric than those who have emotional ones. Even with the much more liberal definition of graduation within eight years of high school entrance, Laura Schifter (Schifter, 2011) found that about $75 \%$ of special education students had graduated by the end of eight years, although that varied from $84 \%$ for students with speech and language impairment and $81 \%$ of students with learning disabilities to $68 \%$ of those with intellectual disabilities, and about $60 \%$ for those with emotional disturbance, autism or multiple disabilities. It would seem that the low rate of high school graduation for special education (as well as for unidentified students), and the low numbers of students with disabilities receiving regular diplomas leaves too many young American adults poorly prepared for work or additional schooling.

\section{Academic Achievement}

In the United States, No Child Left Behind (NCLB), first passed in 2001, required that students with disabilities complete the same testing and assessments as all other students, and be measured against the same proficiency standards as non-identified students to establish whether schools are making "adequate yearly progress." However, because states identify students differently and, in fact, appear to identify very different numbers of students (National Center for Education Statistics, 2015), and because the state tests vary considerably, it is very difficult to assess progress. It is also difficult to chart longitudinal progress because tests and policies, such as test accommodations, change over time. Even using the state data however, it is clear that, while pass rates are improving for both general and special education students, there remains a large gap in pass rates between identified and unidentified students, and the pass rates for students 
served by IDEA are weak enough (in 2003-4, 36\% of schools where students served by IDEA could be counted separately, identified students did not make adequate yearly progress) (Aron \& Loprest, 2012), that there should be concern about preparation for additional schooling, career, and ability to participate fully as citizens.

If, instead, we look at the NAEP, which is the same in all states, and which changes very slowly because it is designed to assess longitudinal progress, we can get a more reliable picture of the achievement of special education students in basic academic skills. While it appears that scores in both reading and mathematics are increasing for students receiving IDEA services, they are still very significantly below those for students not receiving services. For example, in 2009, 64\% of identified twelfth grade students scored "below basic" in reading, while $24 \%$ of non-identified students did so, indicating that students with disabilities did not bridge the gap and make up for differences between special education and non-special education students (National Center for Education Statistics, 2015).

On international tests, the United States fairly consistently scores in the middle of countries taking part in the assessments. Again, it is difficult to compare across countries, because they identify and exclude different numbers of students from taking the tests. Both Sweden and Japan, for example, which identify no more than $1 \%$ of their students, include $99 \%$ in their tests. Among 15-year olds taking the Program for International Student Assessment (PISA), the United States scores well below both Sweden and Japan in mathematics, and with a greater spread between the $10^{\text {th }}$ and $90^{\text {th }}$ percentile levels. In reading, Sweden scores better than either the United States or Japan (Schleicher, 2014). 
Nevertheless, it appears that too many special education students score at levels low enough that they are not fully prepared for either further education or a sustainable job.

\section{Preparation for Jobs, Independence and Continuing Education}

Despite the fact that IDEA requires transition planning for all students served by

IDEA beginning no later than age 16, to help them make the next step after high school completion, data seem to indicate that identified students achieve lower rates of postsecondary schooling, market employment and independent living, and greater rates of involvement with the criminal justice system. Because transition planning is designed to begin at age 16, those students who drop out of school before then generally do not receive the benefit of this requirement. In $2005,46 \%$ of identified students enroll in some kind of post-secondary education within four years of high school, although much of that is in two-year colleges or vocational, technical and business school programs. This can be compared to a $63 \%$ rate for non-identified students.

The United States has not been particularly successful with direct preparation for employment. As of 2003, approximately $30 \%$ of students with disabilities were neither employed, in school, nor in job training programs in the few years immediately after leaving high school, although this percent varied considerably depending on the nature of a student's disability. More than half of those with cognitive deficits were in this group, but only $17 \%$ of those with learning disabilities were (Aron \& Loprest, 2012). The United States, it appears, has not yet developed strong apprenticeship or other job training programs for those students who do not complete high school or who do not continue their academic education beyond high school. Aron and Loprest suggest that, even with improvements in transition planning, coordination and supports to aid in the transition to adulthood and the world of work still need to be improved. They also note that students 
with disabilities are disproportionally represented in the criminal justice system, in alternative education settings and in foster care, where the ability to receive appropriate education and planning for transition to adulthood are particularly difficult.

It appears that there are few studies and little data on such important, but hard to measure, outcomes as satisfaction, social and civic engagement or "quality of life," but, to the extent that these depend on the ability to obtain and retain a job that pays a living wage and provides personal satisfaction, there is evidence that these areas could stand improvement as well.

Fairness: Overrepresentation of Some Groups in Special Education

Among the issues cited in American special education literature is disproportional representation of minorities, especially in classes for students with (educable) cognitive disabilities (Skiba et al., 2008). A secondary issue mentioned in United States literature is overrepresentation of boys, or perhaps underrepresentation of girls, among identified students (Arms, Bickett, \& Graf, 2008). In a longitudinal study of minority representation in special education, Zhang et al. (Zhang, Katsiyannis, Ju, \& Roberts, 2014) found only minor changes over the last ten years, and that minority overrepresentation continues to be a significant problem. Overrepresentation is measured by the extent to which a group is represented in a category of special education (or special education, more generally) as compared to its proportion in the total school population. African-Americans are the most overrepresented in special education, followed by American Indians/Alaskan Natives. Hispanics and Asian/Pacific Islanders are represented at rates lower than Whites. Moreover, African-Americans are more highly represented in the intellectual disability category than any other group, although, in a promising trend, there has recently been a notable decrease in the rate of African- 
Americans classified with intellectual disabilities. There was a significant increase in the percent of Hispanics categorized as learning disabled, which may have resulted from the large increase in the number of English language learners in U.S. schools, more generally.

\section{Financial Incentives and Disincentives}

Another problem with the American special education program is that it appears to create financial incentives that result in overidentification of students as disabled, and, at the same time, lead to less than adequate services for those who have disabilities. Jay Greene (Greene, 2007) observed that the percent of students identified for special education increased from $8.3 \%$ in 1976 to $13.3 \%$ in 2000 , with almost all the increase in those classified as having "specific learning disabilities." He noted that this is a category that is diagnosed more subjectively and is, in fact, much less expensive to remediate than other special education categories. If the percent of the population with disabilities were, in fact, increasing, he asserted, it would be likely to occur across all categories, and not just "specific learning disabilities." He attributed this increase to "perverse incentives" created by special education law, which create financial rewards for school districts for each additional diagnosis, without substantially adding to the costs that the school district would have incurred without the diagnosis. He noted that, for example, if without diagnosis, a school assists a struggling student by providing small group tutoring, it receives no money, but if the student is officially identified, the school receives an allotment in addition to its regular education budget without adding any additional services beyond the ones it was already providing. In addition, the financial incentives lead to underserving students with significant disabilities because subsidies are not based on the nature or extent of services students receive, but only on the number of students. 
Another issue related to economic incentives and disincentives in the United States is the ongoing challenge of increasing the supply of special education teachers, while, at the same time, maintaining or improving the quality of teacher candidates and preparation. While there are many factors that affect the number of available special education teachers, and the rate of teacher attrition, there is reason to believe that teacher salaries, along with working conditions, the nature of administrative support, and the lack of prestige of the teaching profession in the United States contribute to the current and predicted shortage (Brownell, Sindelar, Bishop, Langley, \& Seo, 2002).

\section{Summary}

Despite the fact that the last 40 years have seen great progress in special education in the United States, there continue to be significant problems, with high program costs, as well as personal and financial costs related to the number of administrative actions and court cases relating to special education placement and services, with low graduation rates and weak performance on tests of mastery of the basic skills necessary to succeed and be independent in America's economy. There are also concerns about the way in which Americans prepare special education students for the world of work, and issues related to fairness in identification and overrepresentation of minority students in certain disability categories. In addition, there seem to be a number of financial incentives that tend to lead to overidentification of students as disabled and underserving some of those with disabilities. Further, low salaries, as well as inadequate administrative support, are among the issues that have led to an ongoing shortage of teachers qualified to work with disabled students. 


\section{Commentary on Swedish Success and Challenges in Special Education}

\section{Introduction}

In general, scholars have applauded much about the Swedish special education system, and point to the successes it has achieved, particularly with its emphasis on normalization for all, including those with the most significant disabilities. They have noted the success of the "school for all," in which inclusion appears to be genuine, at least through the compulsory education period, and all students (except for the very small number in special schools) are given access to the full nationally-mandated curriculum, and to the fact that all students can receive assistance, for short or longer periods of time, without the requirement of formal identification or documentation of dysfunction. Commentators have also noted the way Sweden's approach to offering second and third chances to meet requirements has served students who learn atypically. They point to the vast menu of adult education programs and apprenticeships preparing students specifically for the world of work, and to the flexibility in timing so that students are not bound by the standard timetable for reaching educational milestones. They also observe the seamless integration of Sweden's expansive social welfare system with education for students with disabilities. But the system is not without its critics. Many point to the market-based changes since 1990, and suggest that these have led to dilution of the "school for all" concept, to resegregation by economic level or minority or immigrant origin status, and to financial disincentives to full inclusion.

\section{Costs and Legal Burdens}

It has generally been difficult to ascertain the costs of special education comparable to those in the United States because Sweden has identified such a small percent of its population. Therefore, the costs to educate students who have difficulty in school (other than the relatively few with low incidence-infrequent and generally 
serious - disabilities) have been folded into the general education budget. Sweden's spending on education, as a ratio of education spending to GDP, is one of the highest in the world (United Nations Development Programme, 2013). ${ }^{8}$ Any attempt to tease out costs is also complicated by the fact that the Swedish approach is to offer special support to all students who need it, for a short or long time, whether or not they have been officially identified. In addition, some costs that might fall within special education budgets in the United States are included in the social welfare system rather than education. Family subsidies for taking care of disabled children, habilitation services, and job support for older students and adults fall into this category. In addition, significant resources have been committed to the development and utilization of assistive devices, personal assistants, adapted teaching materials, and special transportation, so that disabled individuals can be served within the school for all. However, it seems appropriate to assume that, because so many fewer students are identified, the bureaucratic load and its attendant costs that could reasonably be attributed to special education in Sweden is significantly lower than in the United States.

Under the market-based and more decentralized system that has been in effect since the 1990's, there have been no special funds for special education. Local officials decide on allocations for special education needs in the same way they decide on the use of funds for other programs. The amount of funds the municipalities receive depends, in part, on the number of special education students, but does not require the local

\footnotetext{
${ }^{8}$ It is not clear how to interpret these data because Sweden provides free university tuition, plus a stipend to all Swedes and students from the European Union, as well as a collection of transportation and health benefits in the schools.
} 
educational agency to spend the money on them, making it even more difficult to assess what is spent on special education.

Under Swedish law, parents cannot take legal action related to placement or the nature of special education services, although parents have a fair amount of input into decisions and there are very active groups advocating for educational and social services for disabled students. As a result, there is nothing comparable to costs in the United States for due process hearings and court cases aimed at achieving services or placement for students with disabilities.

Based on these data, it appears that, although Sweden spends a large percent of its GDP on education, it is not clear how much of that goes to serve students with disabilities, other than the group that is identified with low incidence disabilities requiring significant services. It does appear to be the case that administrative costs connected with identification and record keeping are relatively low and legal costs arising from parent challenges are virtually nonexistent.

\section{High School Graduation Rates}

Compulsory education, including about $99 \%$ of all children, is a 9-year program, between ages 7 and 16 in a comprehensive school. Only about $1 \%$ of students attend special schools for students with disabilities. All students completing comprehensive school are admitted to upper secondary school, generally a 3-year program for students aged $17-20$, and about $97 \%$ of all students elect to go to upper secondary school, with relatively few dropouts before completion (Organisation for Economic Cooperation and Development, 2012). For those students who do not pass enough subjects to qualify for a national study program, there is an individual study program, in which students can continue working until they remove any deficits. Fifteen of the seventeen available 
programs prepare students for the world of work and include specific vocational preparation and internships. The remaining two prepare students for continuing academic education. A very small percent of students drop out before completing upper secondary school, and they, too, can continue their education as adults in the free adult education programs offered through the National Adult Education Program, which allow students to complete any requirements for comprehensive school or upper secondary school certificates. Completion of upper secondary school, a level achieved by almost all Swedes, is essentially equivalent to high school, plus a year or more of college. Students can then earn a university diploma after two additional years, or a bachelor's degree after three years. Thus, completion of an upper secondary program prepares students well for jobs in the market economy.

On the metric of high school graduation rates, Sweden has been very successful, and commentators have observed that strong social supports, flexibility with respect to time and acceptable alternate routes to secondary school completion are among the elements that have made this success possible, despite prescribed courses and fairly stringent requirements for knowledge and skills before high school graduation.

\section{Academic Achievement}

Swedish scores on the international PISA and TIMSS examinations are very close to those in the United States (Schleicher, 2014), and at about the midpoint for the OECD (Organisation for Economic Cooperation and Development, 2012) although Sweden excludes fewer students as disabled, because they are not identified, with similar numbers of students scoring below the $10^{\text {th }}$ percentile or above the $90^{\text {th }}$. It is difficult to interpret these numbers, given the much higher secondary school completion rates in Sweden, and the fact that completing secondary school requires passing nationally administered 
achievement tests and all students take courses in eight core subjects: Swedish (or Swedish as a second language), English, mathematics, natural science, civics, the arts, physical education and health, and religious knowledge, which are intended to prepare them for higher education, even if they are in a vocational stream.

Another reason for the apparent discrepancy between very high graduation rates and mediocre scores on international examinations is that international tests examine students based on performance at a given age, rather than on the level of achievement they can attain, given enough time. British researcher Harold Huddart (Huddart, 1981) observed the absence of strict timetables, especially for school examinations. He noted that, with extra time, a substantial number of physically or mentally disabled students could achieve a level of skill and knowledge that is comparable to that achieved by students who are not considered to be disabled.

Sweden's scores are average for OECD nations and similar to those attained by the United States, so this area is not one of great strength for Sweden, but it achieves these scores by including and by identifying a relatively small number of students beyond the small percent of students with low incidence disabilities.

\section{Preparation for Jobs, Independence and Continuing Education}

Commentators point to Sweden's very strong commitment and performance in this area as the hallmark of its success with individuals with disabilities. In the sense that scholars have defined one of the markers of "success" in special education as the ability of individuals with disabilities to lead as normal and productive life as possible, including, if possible, holding a job and participating in social and civic life, Sweden can count as one of its successes its very early commitment to "normalization." Both internal and external scholars of Swedish special education have commented on the degree to which 
Sweden has approached true normalization for the great majority of its citizens with disabilities, as a result of a combination of commitments to both social welfare and occupational support.

As early as the 1970's, before the passage of the first special education laws in the United States, when most individuals with visible disabilities were institutionalized or at least taught in separate schools, Americans Mary Jane and Kenneth Clark (Clark \& Clark, 1970) observed the Swedish approach, which included living and eating with family or in family-like communal settings, privacy, working, if possible, in a setting separate from their home, having options and a choice of how they spend time and money, and the ability to maintain relationships with their family and travel or have visitors.

Also, as early as the 1970's, American researcher Nicholas Castricone (Castricone, 1974) observed that Sweden's special education programs were based on two factors, its social welfare system and the "normalizing principle." The social welfare system, he observed, provided services to the disabled and their families free or at very nominal costs. Normalization significantly affected treatment of all students with disabilities and especially those with mental retardation.

Similar results were reported by another American, George Bereday (Bereday, 1980), several years later. He noted that Sweden was at the forefront of providing maximum care for students with severe mental and physical disabilities within normal schools and in an integrated setting, and that Sweden was committed to providing assistive devices to do so. Even in residential facilities for the most severely affected, every effort was made to create "normalcy." 
Harold Huddart (Huddart, 1981) reported on his observations on Swedish vocational education for students with disabilities. He particularly noted a substantial commitment to providing staff to assist and supervise young people with disabilities so that they can live in non-institutional settings. In addition, he saw the use of a wide variety of communication aids and other technology to aid students' ability to participate in the regular classroom. He also noted that disabled students participated in the work experience program that was an integral part of vocational studies for all students. He commented that it appeared to be a basic principle that efforts be made not only to deinstitutionalize young people with disabilities, but to place them, whenever possible, in open market employment, and that there was great reluctance to allow students to leave school without job prospects. He also noted that Sweden has a "Promotion of Employment Act," which grants priority to handicapped applicants for employment, and which was passed after consultation with both trade unions and employers' organizations, with the state paying from $25-90 \%$ of the disabled individual's salary, depending on the degree of disability.

The Swedish commitment to aiming for full, market-based employment for the greatest number of students with disabilities was also noted by the Organisation for Economic Cooperation and Development (OECD) (Organisation for Economic Cooperation and Development, 1983). For virtually all individuals with disabilities, a regular job is seen to be "the threshold of personal achievement"; (p. 12) individuals with disabilities desire to work even when pensions and other financial support are adequate. Swedish policy continues to assume that the overwhelming majority of those labeled as "disabled" are likely to be able to hold a job. This sometimes requires a financial 
commitment by government to supplement salaries, at least during training, and a commitment to training in social skills, citizenship, family living, home management, and basic financial skills, in addition to specific job training and general education at an appropriate level.

\section{Fairness: Overrepresentation of Some Groups in Special Education}

In the past 50 years, Sweden's educational policy, combined with its highlydeveloped social welfare system has succeeded in creating a true school for all, which maximized equality of educational experience across regional and social class lines. By international standards, Sweden has been extremely successful in minimizing differences and honoring and including many dimensions of diversity, including the wide range of abilities and disabilities. But, several commentators (Arnesen \& Lundahl, 2006), (Brodin \& Lindstrand, 2007), (Antikainen, 2006) ${ }^{9}$ voiced concerns that, since the educational reforms of the 1990's, which introduced marketization (competition among schools, and with an increased private school presence) and decentralization of the education system, this movement toward equality has stopped, and separation by socioeconomic level, immigrant status, and disability was growing.

Girma Berhanu (Berhanu, 2008; Berhanu, 2010; Berhanu, 2011) wrote about the effects of 20 years of policy since 1990, described by him as "guided by a neoliberal political agenda," on the Swedish values of equity, diversity and inclusion that underlie the school for all. His primary argument was that, despite continuing allegiance to policy goals favoring equity and inclusion, policy changes since 1990 resulted in increased marginalization and segregation of ethnic minorities, students from socially

\footnotetext{
${ }^{9}$ The critiques expressed by these authors are discussed in greater detail in Chapter IV, which discusses special education in Sweden.
} 
disadvantaged backgrounds, students with special needs, and immigrant students. Decentralization, beginning in the 1990's, also led to greater differences in resources available to individual students, schools, and municipalities.

Berhanu observed that, in the last century, inequalities, as measured by the degree to which parents' socioeconomic status and educational attainment affected students' educational outcomes, had decreased, and asserted that Sweden had been a leader in combining equity with high levels of economic efficiency. Government policies, including high spending on social welfare, health and education, and imposing high levels of taxation, continued to be strongly supported by public opinion. Berhanu noted that studies by the OECD concluded that, since the 1990's: (1) numbers of students identified with special needs increased; (2) numbers of students attending private schools increased dramatically; and (3) differences among schools on measures of academic achievement increased, along with between-school variance in socioeconomic and minority status.

Berhanu also observed the major educational changes caused by massive immigration in the last 20 years, which changed Sweden from a largely homogeneous society to one that is multicultural and global. As of 2010, approximately $20 \%$ of the Swedish population had an "immigrant background" (being born outside the country or having one parent who was born outside the country). This rapid change resulted in increased ethnic segregation in housing, particularly in cities, and led to an increase in school segregation by educational background and immigrant status. In Berhanu's view, it also resulted in overrepresentation in special education programs of students of 
immigrant background and students with low educational and economic status backgrounds.

The majority of students of Swedish background who are currently placed in special education schools have clear medical or functional disabilities (low incidence disabilities), while a much larger percent of those of immigrant background have "high incidence" diagnoses, such as speech and language problems, concentration problems, or behavioral problems. Another way to look at the same phenomenon is that immigrant students are represented in low incidence disabilities in about the same ratio as in the general population, but are significantly overrepresented in the high incidence, more subjective disabilities.

Mara Westling Allodi (Allodi, 2007) observed that, among all students, about one-quarter did not achieve educational requirements at the end of compulsory school, and about one-tenth performed poorly enough that they did not qualify for any national secondary school program, although they were able to enter upper secondary school and work to eliminate any deficits in an individualized program. Students with non-Swedish background, particularly boys, were overrepresented among those who did not perform sufficiently well to enter a national program. Despite the fact that Sweden has eliminated all costs and other economic obstacles to post-secondary education, students applying for post-secondary education continue to be significantly more often from families with a higher academic and economic background.

Despite these critiques, it is clear that fairness is one of the areas of great success for Sweden's special education program. The promise of a school for all has largely been fulfilled, although some commentators point to recent retraction. While there is some 
overrepresentation of minorities and immigrant groups among those identified as needing special education, the total number of students so identified is still so low, that it is a minor effect, at least compared with the United States.

\section{Financial Incentives and Disincentives}

In the years since 2005, research and commentary have centered on whether education of students with "school difficulties" has approached the inclusive and generally-accepted ideals of a school for all. Several commentators suggest that, particularly since the reforms of the 1990's, the system had not gotten closer to full inclusion and that, in many cases, inclusive education appears to be decreasing and segregation, both within schools and by placing students in special schools and classes, is on the rise. In addition, according to the commentators, there are clear impediments to increasing inclusive practices resulting, first, from economic constraints and, second, from the relatively new competitive environment, which allows parents to choose schools based, in part, on published reports of schools' achievement scores.

In the 1990's, with the increased move toward decentralization of education, responsibility for schooling moved from the state to municipalities and schools. Schools became responsible for finding ways and managing budgets to meet the goals. Neither Swedish school law nor regulations mandate how students are to be identified for special education. Thus, the process for identifying students to receive special services came to rest at the municipal and school levels.

Jane Brodin and Peg Lindstrand (Brodin \& Lindstrand, 2007) observed that, under this new system, official diagnosis carries with it money to pay for extra support. The result is a risk of overdiagnosis to increase financial support, a risk they believe is strongest for what they call the "acronym children," those with attention deficit disorder 
(ADD), attention deficit hyperactivity disorder (ADHD), and deficit in attention, motor control and perception (DAMP). Several other commentators echoed this concern, that under a decentralized system, economic incentives pushed school districts away from the long-held and widely shared vision of a school for all to a resegregation, often by class and immigrant status, along with disability.

Anne-Lise Arnesen and Lisbeth Lundahl (Arnesen \& Lundahl, 2006) looked at the role of education policy related to minority and special needs children in the context of the modern welfare state. A uniform, free educational system, the school for all, was expected to result in a cohesive society characterized by equality and justice, and universal education was believed to contribute to full employment, social acceptance and a healthy society. While the politics of education always involves both economic and social welfare motives, in the last two decades, economic concerns have been increasingly influential in both welfare and education policies.

Teaching in Sweden is a prestigious occupation, and there is competition for university slots in both general and special education, so there do not appear to be any significant economic disincentives to maintaining an adequate number of well-prepared special education teachers.

While there has been recent criticism about the growth of economic disincentives for maintaining the hard-won level of equality and fairness achieved by the creation and growth of the school for all, it is in the context of a society that has strong social and political values favoring equity. As with the metric of fairness and overrepresentation, even with the recent disincentives, the Swedish system continues to be committed to minimizing these challenges and maintaining the promise of the school for all. 


\section{Commentary on Japanese Success and Challenges in Special Education}

\section{Introduction}

Japan's education system has been consistently praised by scholars and

commentators for the high achievement of its students, particularly on international comparisons and the fact that few students score at a low level. They observed that this has been consistently true despite a very low percentage of students identified for special services and, until very recently, very few special services within the schools. Observers have suggested that this success has been due to a strong cultural commitment to education and a belief in perseverance over "ability," which leads to persistent effort.

\section{Costs and Legal Burdens}

Except for the costs of educating the approximately $1 \%$ of identified students, almost all with low incidence disabilities, generally in separate classes or schools, or in some cases, in resource rooms within public schools, Japan has, at least until recently, invested relatively little money in special education. Schools generally did not have special educators, counselors or speech or occupational therapists on staff, and there was little bureaucratic load. Despite major changes in law (Ministry of Education, Culture, Sports, Science and Technology (MEXT), N/A) and some real changes in the discourse surrounding special education since 2005, changes at the school and class level have been very slow (Goto, 2008; Kayama \& Haight, 2012). The great majority of students with significant disabilities are still served in separate settings, with a few small experiments in "integration," and the great majority of those with high incidence disabilities are still served in regular classrooms, with relatively minimal increases in available supports.

Even when asked directly, Japanese educators tend to identify far fewer children as having high incidence difficulties, such as attention deficit disorder (ADD), learning disabilities (LD), and high functioning autism, and, when they describe classroom 
difficulties, tend to blame them on teacher inadequacies or lack of persistence (Haynes, 2000).

As of 2012, there were only 67 court cases relating to special education in the entire period since World War II, and most of those were about whether students with disabilities could attend a regular school as an "exception" to the assumed placement in a separate setting (Nagano \& Weinberg, 2012). The cultural preference for conciliation rather than confrontation has meant that the legal system is rarely used for special education complaints and that, therefore, legal costs are not a significant burden.

This suggests that, on the basis of a cost-benefit analysis, Japanese educators have managed to produce excellent results with a relatively low expenditure on special education.

\section{High School Graduation Rates}

All students (except the approximately $1 \%$ with low incidence disabilities) are grouped heterogeneously for compulsory grades 1-9. Students do not repeat grades, because that would separate them from their peer group. In ninth grade, all students take examinations that determine where they go to high school. The high schools are ranked from most to least academic, so at entrance to high school, for the first time, students are separated according to academic performance. Virtually all students completing compulsory school (estimated at 95\%) also attend and graduate from high school (upper secondary school), which includes grades 10-12, and includes both highly academic schools and technical schools (Ellington, 2005b). There appears to be no significant problem with students dropping out, although there are references to a small number of students who are "school refusers." About half of each cohort completing the upper secondary level continues to some form of higher education, with nationally-run 
universities being the most prestigious, although there are private colleges as well, and a relatively small junior college presence. About $25 \%$ of each cohort begin a four-year bachelor's degree program, and virtually all of those complete them.

Given that so few students are identified as being disabled, combined with the very high upper secondary school graduation rate, it would be reasonable to assume that the great majority of students who, in the United States, would be identified as having high incidence disabilities, succeed in graduating from upper secondary school.

\section{Academic Achievement}

Japanese students regularly score near the top of the pack of nations on international exams, although they score better in mathematics than in reading/language (Schleicher, 2014), and there has recently been much internal Japanese commentary about Japan's minimal slide in the rankings from the very top to being among the strongest countries. There has been much commentary about reasons for Japan's success in general education, centering on such things as the nature of the curriculum-clear, sequential and non-repetitive, with greater depth and less breadth than in the United States, the nature of teacher preparation and cooperation, using lesson study, for example — and the cultural expectation of hard work and emphasis on educational credentials, but very little in the literature about why it has been apparently successful with students who might be presumed to be identified with higher incidence disabilities in the United States and elsewhere.

In one of the few articles addressing how Japanese educators achieve success with low achieving students, John Woodward and Yumiko Ono (Woodward \& Ono, 2004) found that some aspects of diversity were addressed before children enter elementary grades, through preschool programs that emphasize socialization into school behaviors, 
such as group work, and membership in the class community. In elementary schools, high and low performing students are consciously grouped together in work groups, assuming that slower and faster learners would both benefit. They also noted that two important ideas are emphasized: gamburu (effort) and gaman (persistence). Rather than allowing slower students to limit the curriculum or placing them in alternative classes, they are encouraged to put in extra effort, and Japanese teachers often work with students who need extra help outside the normal class setting. At the middle school level, where the pace of instruction and focus on examinations increases, $j u k u$ (private "cram schools" supplementary to the public school) become more significant parts of education. Juku serve a variety of functions, including preparation for examinations, enrichment and remediation.

High levels of academic achievement are evidence of Japan's success at educating the great majority of its young people and, given the small number of students who are given extra assistance, a major accomplishment. There is evidence that strong cultural support for education and persistence in the face of challenge are factors in this success.

\section{Preparation for Jobs, Independence and Continuing Education}

Because so few students are identified, and virtually all of those who attend regular schools complete some kind of upper secondary education, it is difficult to track how students with high incidence disabilities do in terms of employment and continuing education or to measure Japan's success in providing meaningful market-based jobs to students who would have been identified under American special education law.

Nevertheless, Yui Murakami and Heinz-Dieter Meyer (Murakami \& Meyer, 2010b) observed a significant change in recent years leading to decreased institutionalization and increased presence in the community for students with lower 
incidence disabilities. Murakami and Meyer suggested that this change has come, in part from the rise of grass-roots organizations pushing for greater inclusion and increased services, although a percentage of those with significant disabilities still return to their families and continue to be cared for at home after finishing their school years. There is no evidence, however, for students with significant disabilities, that there has been any move toward what the Swedes would call "normalization."

Several scholars have noted that, despite education law requiring universal education, and the availability of special schools and classes for those with significant disabilities, there remain approximately one million children, known as kikikomori, who do not attend school and are therefore not served by any special education program. Many of these students would likely be candidates for appropriate special education. The problem of so many children outside of the school system has received both Japanese and international press coverage, and is often attributed to Japan's strongly conformist social structure, which emphasizes obedience, discipline and group harmony (Borovoy, 2008). This observation suggests that, despite Japan's apparent success with high achievement and high graduation rates, that there are students who are not being fully prepared for jobs, independence and citizenship.

While students with low incidence disabilities and the so-called kikikomori children remain outside the mainstream of Japanese work life and culture, it appears that Japan has been mostly successful in preparing the great majority of students for full participation in society, both by imparting basic academic skills and by the strong emphasis on socialization and behavior in Japanese schools. 


\section{Fairness: Overrepresentation of Some Groups in Special Education}

As compared with the United States, which adopted a "human rights approach,"

(Murakami \& Meyer, 2010b) Japan's special education system continues to be based on a medical model, where individuals and/or their parents defer to authorities, who determine where they go to school and what they learn based on a medical diagnosis. Parents tend to accept the word of medical or educational experts and there is virtually never any legal action relating to a student's placement. Diagnosis of low incidence disabilities tends to be non-controversial, and not subject to overrepresentation by population subgroups, as in the United States and Sweden.

Unlike in the United States, there is little talk about the rights of either students or parents (Nagano \& Weinberg, 2012), and the concepts of "normalization" and "equal access" used in the United States are rarely used. Nagano and Weinberg also suggest that there is a significant problem of underidentification of high incidence disabilities, even several years after the 2007 law defining high incidence disabilities. The number of students who could benefit from special services within the regular classroom, they assert, is probably substantially higher than the number of students currently identified, based on the fact that the total percent of students identified as needing some kind of assistance is far lower in Japan than in most other developed countries, including the United States.

Japan has several ethnic minority groups (World Population Statistics, 2013). Although the largest ethnic group by far is the Yamato Japanese group, which makes up about $98.5 \%$ of the population, there are several other groups: the Korean ethnic group, making up about $1.5 \%$ of the population; people of Chinese heritage; and several groups of indigenous people. While there is evidence that diversity is increasing and that 
minority groups are not always treated well, there has been little discussion of their overor under-representation in special education.

\section{Financial Incentives and Disincentives}

There has been little discussion in the literature paralleling the discussions about financial incentives and disincentives to identification and provision of special education services in Japan. This may be because Japan's educational establishment remains centralized and does not appear to provide funding at a school or district level based on the number of students identified as needing special assistance.

In Japan, as in Sweden, teaching is a prestigious vocation with lifelong employment, pay at a level commensurate with jobs in business, a very low turnover rate and almost no teacher shortages. Low birthrates and a shrinking school-age population increase competition for teaching jobs (Ellington, 2005b). Teachers in special education classes or schools are paid an additional stipend of 6-8\% above their regular teacher salaries, although only one-third of such teachers have a special education license, and most of those have been prepared to teach in the separate schools for low incidence disabilities. As of now, very few teachers have been trained in working with students in regular schools with high incidence disabilities. Only 7-8\% of Japanese teachers hold master's degrees; there is no extra stipend for additional education, only for years of service. 


\section{Chapter III: Methodology}

This chapter is a discussion and justification of the research methodologies of this study, along with a statement describing the researcher's educational background, positionality, and possible biases. It also contains a discussion of known and potential limitations of the study.

This dissertation is a comparative case study of special education in Sweden and Japan, using elements of historical analysis to shed light on the development and changes in special education over the last 40 years. Each case was developed using primary and secondary sources, primarily scholarly articles, books, websites and other documents relating to each country's development of policy and practice in special education. Materials were collected in a systematic way, aiming at a balanced and complete picture of the development and current state of special educational practice in each country. The resulting materials were reviewed and analyzed in order to look at patterns and trends for each country, followed by a comparison of data from the two countries. The results were

then used to determine what we, as Americans, might learn from the two cases to apply to our policy decisions about special education in the United States. The sections below describe, in greater detail, the methods of materials collection, analytical approaches, and safeguards to ensure as accurate an interpretation as possible.

\section{Comparative Case Study}

The term "case study" has often been used as a synonym for ethnography, but case study and ethnography should not be confused. Some, but not all, case studies may use ethnographic approaches. John Elliott and Dominik Lukes (Elliott \& Lukes, 2008) 
define "educational case study" as a "form of inquiry into a particular instance of a general class of things that can be given sufficiently detailed attention to illuminate its educationally significant features." (p. 88) In this dissertation, the two cases are special education policy and practice in Sweden and in Japan. An educational case study, Elliott and Lukes suggest, can use any of a number of methods, including but not limited to ethnography, but which should be judged by "fitness for purpose." A comparative case study is one that looks at two or more cases, and then looks for similarities and differences between or among them in order to draw conclusions about common factors that may result in a particular educational outcome.

Case studies have been used in education since the early 1900's, and were initially used to help decision-makers understand how a particular change or reform acted in a specific educational context. They have also been widely used as a qualitative evaluation technique for educational programs and as an alternative or supplement to more quantitative studies.

Among the seminal articles about the use of case studies in comparative education research were ones by Stenhouse in the late 1970's (Stenhouse, 1979). Stenhouse advocated qualitative case studies as a complement rather than an alternative to quantitative studies using statistical data, stating that case studies are able to contextualize the findings of studies of samples, and can thus help improve the judgment of educators as they make practical decisions. He argued that overemphasis on quantitative results from sample studies marginalizes the importance of professional judgment. Nevertheless, he raised concerns about verifying interpretations and the ways they might be used by others who might be operating beyond the boundaries of the cases 
examined in a study. In Stenhouse's view, researchers could obtain more widely generalizable knowledge by aggregating cumulative knowledge from a number of case studies. He thus advocated clear procedures for verifying case studies, including guidelines for case records, and use of similar factors in multiple specific cases to produce generalizations. This approach is, for him, not unlike the techniques used in historical study. A comprehensive, historian-like approach to data collection, retention and dissemination provides for the possibility of multiple interpretations by different researchers, and allows us to account for the fact that the data collected are complex, and embedded in a particular situation in which they can be interpreted. In case study research, as distinguished from sample studies, Stenhouse argued that the relationship between the cases studied, or an aggregation of cases and the population to which inferences might be generalized is always a matter of the researcher's insight and judgment. Other authors, such as Crossley and Vulliamy (Crossley \& Vulliamy, 1984), suggested that case studies, by focusing on the complexities of education can be important factors in modifying educational policy and in developing theories about educational systems.

\section{Methods and Materials}

To address the research questions outlined in Chapter I, this dissertation uses historical and government documents, research articles, commentaries and opinion pieces from the object country, as well as by observers from other countries, published educational data and websites. In the case of Japan, these sources were supplemented by personal interviews and observations, taken while the researcher was a Fulbright Teaching Fellow in Japan, which, although ten years old, helped to give context as well as detailed information about the status of special education in 2005. The sources were 
specifically chosen to provide information about the cultural, historical, political/policy and legal factors underlying the Swedish and Japanese general and special educational systems, and the factors that may lead to each country's apparent success in educating the great majority of students to a level that represents an ability to be successful participants in the financial, occupational, civic and social fabrics of their country's culture.

\section{Materials Collection}

An exhaustive search of literature on general and special education in both Sweden and Japan was completed using the EBSCO Databases: (Education Research Complete; ERIC; Education Index, Retro; Education Full Text; Psychology \& Behavioral Sciences; Academic Search Complete; and SportDiscus) using the University of Virginia portal, and entering a variety of keywords designed to identify all articles of potential relevance. The collection of data was undertaken starting in 2005; additional searches were done several times during the course of the research, most recently in 2013. Additional research on theoretical and methodological issues was done through 2015 . In order to find new articles as they appeared in the literature, the search was updated monthly through the EBSCO update feature. The researcher read abstracts of all of the identified references and selected those primary and secondary sources that appeared to be significant and relevant to the research questions. The researcher also searched Virgo and ProQuest Dissertations \& Theses for additional sources, and to ascertain that the work described in this dissertation does not duplicate or replicate other studies. All references were imported into RefWorks, where they could be accessed for citation in this dissertation. While the researcher attempted to be exhaustive, she is aware that, by the choice of search engines, search terms, and in her review of abstracts, she was making decisions that might bias the nature of the research included in this study, and thus the 
conclusions drawn. As discussed in greater detail in the section on study limitations, the thoroughness of the search was further bounded by the fact that the search was limited to articles originally written in or translated into English. The raw materials selected included primary and secondary sources, articles from peer-reviewed journals, as well as opinion articles.

Literature specifically about either Sweden or Japan was organized chronologically in order to understand changes in policy and practice and the forces affecting these changes. Literature supporting study justification, research methods, analyses, discussion and conclusions was organized by topic. Copies of all articles were retained for possible additional review by the researcher, or by reviewers or users of this research. The researcher then read and wrote a rich summary of each relevant article.

\section{Materials Management and Analysis}

In choosing this technique and set of materials to address the research questions, the researcher followed the guidance of Marshall and Rossman (Marshall \& Rossman, 2006), who asserted that the choice to base research on gathering and analyzing documents and archival records must be linked to the research questions and the conceptual framework of the study. They observed that reviewing documents and journal articles is a strong technique for documenting major events, crises and conflicts, and it allows access to distant participants and information. It provides information that is easy to manipulate, manage and categorize for analysis, and assists in analysis, validity checks and triangulation. Among the important advantages of basing a study primarily on documents is that it is non-reactive - it can be conducted without disturbing the setting in any way. It is relatively clear and transparent to readers; information can easily be checked and reviewed by anyone who wishes to evaluate or extend the research, and the 
reader can easily review the basis for analysis and the thought process and care with which the analysis has been conducted. However, Marshall and Rossman cautioned that it is important for the researcher to approach documents with skepticism and be fully aware that analysis of the content of written materials entails interpretation. In particular, research based primarily on reading of documents and research articles is subject to misinterpretation due to cultural differences between the writer of the article and the researcher, and the quality of analysis depends on the researcher's skill.

Marshall and Rossman also suggested that the use of a chronological approach, that is, focusing on the development of and changes in policy over a relatively long period of time as a way to understand the forces and decisions that created the current set of policies and practices, facilitates discovery of nuances in culture, provides for flexibility for organizing information, allows putting in formation in context, provides large amounts of information quickly, and is relatively easy to manage and administer. However, Marshall and Rossman noted that such a chronological approach is also subject to possible misinterpretation due to cultural differences. They pointed out a natural tension between contemporary and historical interpretations of events, and that both contemporary and historical texts may be influenced by the social contexts in which they were written.

In the sense that the articles used in this study represent the use of a number of different approaches — historical, cultural, political — and were written by researchers from the target country as well as from other countries, and with a variety of personal, cultural and affiliation biases, they support each other and provide a better picture of the state of special education in Sweden or Japan than could have been provided by any one 
of them, effectively triangulating the data. It is intended that reviewing such a rich collection of data and documents would ameliorate some of the weaknesses that might otherwise attach to a study based on reviewing documents.

\section{Analysis}

The analysis in this dissertation is a comparative and interpretive analysis of the two cases, describing education and special education in Sweden and Japan. It develops generalizations about the historical, cultural, policy and legal factors affecting special education in the two countries and leads to conclusions about those factors that have influenced success in each country, and that American educators might learn from in their quest to improve special education in the United States.

Materials were collected, organized by country and date, and subtopic (history, culture, policy, legal), and reviewed and summarized. These summaries were then used to write Chapters IV (Sweden) and V (Japan). These summaries served as the basis for generating common themes. These themes are discussed in the Discussion and Analysis chapter of this dissertation. By arranging the materials for each country chronologically, the data make possible a historical analysis, which looks at the way current policy developed, and at the way culture and cultural changes affected that policy.

Marshall and Rossman (Marshall \& Rossman, 2006) used the term "analysis" to include three separate activities: description, analysis and interpretation. They broke down the analytical process in qualitative studies into several steps. In the current study, because it is based primarily on written materials - documents, research articles, critiques, commentaries, etc. - some of the steps take on greater significance than others, whereas in different studies, which may be based primarily on interviews and observations, a 
different group of steps may be most significant. For each of the steps, I have indicated how I applied their principles in this dissertation.

Organizing the Data. Materials were organized chronologically by country, and labeled so that articles and other documents could easily be revisited by other researchers. All data were entered into RefWorks bibliographic software and separated into folders for easy retrieval.

$\underline{\text { Immersion in the Data. }}$ Materials were read and reread, with careful attention to how the written materials were reduced and summarized. All articles deemed relevant to the study were read carefully and summarized. Sections were written and rewritten as new or newly discovered information emerged.

Generating themes. This step required comprehensive awareness of the body of material, focused attention on those sources, and openness to new ways of categorizing and interpreting information. It included identifying significant themes, recurring ideas or language and patterns of belief. Marshall and Rossman noted that this process may run the risk of imposing meaning that might reflect the researcher's world more than the world under study. This, the authors said, is probably the most intellectually challenging phase of materials analysis, and the one that makes it possible to make the entire study coherent.

Themes were generated and often modified subsequently in the reading and summarizing of articles, in the process of organizing, writing and editing each of the country chapters (Chapters IV and V), and in the process of writing the Discussion, Analysis and Conclusions chapter (Chapter VI). 
Writing analytic memos. The researcher took careful notes, writing comments and questions for discussion during the reading of the articles, during the writing of Chapters IV and V, and during discussions with colleagues and advisors. These memos were later used in preparing the Discussion and Analysis sections of the dissertation.

Offering interpretations (which Marshall and Rossman referred to as "telling the story"). This step brings meaning and coherence to the themes and patterns that are observed and creates a "story line" that makes sense, based on the information garnered from the sources. This is the content of Chapter VI (Discussion and Analysis). Chapter VI also offers suggestions for further study, designed to extend or test the interpretations described that chapter.

Searching for alternative understandings. The researcher evaluated the plausibility of her developing understandings, and considered other possible interpretations of the sources. In this study, this took the form of attempting to understand why, for example, Japan has resisted adopting changes in special education practice even though it has altered its education laws to accommodate change, and why Sweden seamlessly adopted marketization and school choice, although these concepts seemed to clash with ongoing cultural practice. The researcher came to the interpretations presented in this dissertation after considering reasonable alternatives.

Riessman (Riessman, 1993) suggested several criteria for assessing the quality and "validity" of an analysis: (1) Persuasiveness and plausibility_-Does the conclusion make sense? Riessman asserted that arguments are most persuasive when they are supported by the available evidence and when the analyst has considered alternative interpretations. (2) Correspondence-Would the authors of the studies used in the 
analysis believe that they were accurately represented? In a document-based study, this can occur only by referring back to the texts, virtually all of which are in the public domain and fully available for scrutiny, or by communicating directly with those authors whom it is possible to contact. (3) Coherence-Has the researcher persuaded the reader that her interpretation is sensible? Riessman advocated making descriptions as "thick" as possible. (4) Pragmatic use-Could this study become the basis for another researcher's or policymaker's work? Validation of pragmatic use in the current research comes from whether the interpretation presented here furthers understanding of the successful approaches to special education policy in Sweden and Japan, and how they might be applied to American education.

As described by Marshall and Rossman and also by Lincoln and Guba, in their classic treatise on Naturalistic Inquiry (Lincoln \& Guba, 1985), in a qualitative study, without statistical rules to guide decisions about such things as reliability, validity and objectivity, we have to ask alternate, but parallel questions about credibility, transferability and dependability. Lincoln and Guba referred to answers to these questions as establishing "truth value."

Lincoln and Guba proposed four alternative constructs for qualitative research, parallel to the conventional positivist paradigm $^{10}$ : internal validity, external validity, reliability, and objectivity:

Credibility (parallel to internal validity). Lincoln and Guba suggested techniques to increase the likelihood that findings and interpretations would be credible. Of these,

\footnotetext{
${ }^{10}$ According to the positivist paradigm, knowledge depends on quantifiable observations that lend themselves to statistical analysis. It views the world has having discrete, observable events that interact in an observable and regular manner. The researcher is always considered to be independent of the study, and therefore does not influence its outcome. Adapted from http://research-methodology.net/researchphilosophy/positivism/.
} 
prolonged engagement, persistent observation (of the literature) and triangulation are all applicable to this study. The exhaustive search for materials, the large volume of documents and research studies reviewed for this dissertation, and the care taken with each of them are believed to satisfy the first two criteria. Triangulation is the act of bringing more than one source of information to bear on a single point. The fact that the sources represented came from a large number of different articles viewing Swedish and Japanese education from different points of view — as teachers, administrators, researchers, government and international officials, as well as government documents and laws - offers a measure of triangulation about both the data and interpretation of the information.

Transferability (parallel to external validity). A qualitative study's transferability or generalizability to other settings is problematic. Generalizing qualitative findings to other populations is often seen as the weakest link in qualitative research. It is the researcher's responsibility to provide the references to source material and "thick description" that makes it possible for subsequent researchers to make transferability judgments based on the specific time and context of their research. In this study, transferability is a desired outcome because the researcher hopes that what she has learned about factors that seem to result in good outcomes for special education students in Sweden and Japan can help to guide American educators to improve special education outcomes in the United States and increase the number of students identified as eligible for special education who complete their education ready for success in work, citizenship and participation in social life. 
Dependability (parallel to reliability). The positivist notion of reliability, a measure of the degree to which one would get the same result upon repeating an experiment in exactly the same way, assumes an unchanging universe in which an inquiry could be replicated. One could assume that a study would not be credible without being dependable, so that a researcher would not have to confirm dependability independently, if it could be established to be credible. In the current research, where all sources are transparent, through bibliographic references that are easily acquired for verification, demonstration of dependability is relatively straightforward.

Confirmability (parallel to objectivity) asks whether the findings, interpretations and inferences of the study could be confirmed by others and whether a reader can see how the inferences were made. Lincoln and Guba asserted that the logic and interpretation involved in qualitative inquiry can be made (somewhat) transparent to others, thereby increasing the strength of the assertions. To the extent that, in the current study, both the source materials and interpretations are as transparent as possible, all reasonable efforts have been made to ensure confirmability.

\section{Researcher as Instrument}

As both a teacher and a parent, I became interested in issues surrounding policies related to how schools deal with students who do not fit the typical mode and pace of learning, either in general or in some aspects of their interaction with schools. These students - including those who struggled in school, or were "advanced" in some areas, or "dually exceptional," or had difficulty with some educational tasks, but not others-did not necessarily fit the definitions used in the United States that would qualify them for special services under the Individuals with Disabilities Education Act (IDEA), but nevertheless fell into gaps between what was offered or made available in terms of 
educational programs and assistance and what they "needed" to benefit fully from their education. Twenty-plus years of teaching in a small, independent progressive school, which tends to draw a fair number of "able" students, who nevertheless are hampered by a variety of hurdles to their learning, further increased my interest in the subject of this study. Because, as an independent school, we are not bound by the special education laws and funding formulas that apply to the public schools, we are often able to furnish learning accommodations and supports that some of these students would not qualify for in public schools under current law, and at the same time expose them to the full, highly enriched curriculum normally available to all students.

I have always had a strong interest in policy and politics and how they play out in both formal education laws and regulations, and in actual classroom practice, and that is what led me to the study of Social Foundations of Education. As part of my study at the University of Virginia, course work in Comparative Education ignited my interest in finding out how other countries and cultures approach their decisions relating to education of students who do not fit the typical mold. I hope that this research provides avenues for broadening our horizons and looking further at ways to educate students who learn differently, and that it provides a window for looking at United States policy and practice in a critically constructive way.

When I began my coursework in the doctoral program in Social Foundations of Education at the University of Virginia, I chose to focus my work, beyond the basic courses in Social Foundations, in three major areas: policy; exceptionalities; and statistics, research and assessment. In order to understand more fully the cognitive and achievement tests that form the basis of identification of students in need of special 
education in the United States, I took a number of courses in statistics and assessment not typically taken by students in the Social Foundations program. In addition, as a mathematics teacher (and former chemist), I bring to that study a strong background in mathematics and statistics. In October 2005, I had the opportunity to spend three weeks in Japan as a Japan Fulbright Memorial Program Scholar. During that time, I focused my attention on the way educators in Japan approached students with learning differences and other disabilities. I observed classes, including general education classes at elementary, junior high and secondary levels, special education classes within regular school buildings and "resource rooms," and interviewed teachers, parents, principals and professors of education. While many things may have changed since my visit (including a new law enacted in 2007, which recognizes "minor" disabilities, such as learning disabilities, ADD/ADHD and high functioning autism), my visit provided a context for my understanding as I read articles from before my visit as well as subsequent publications.

As a researcher, I am not wedded to any particular model of special education provision, either that of the United States, or of any of the other countries studied, although I carry a strong belief in "education for all" in its broadest sense—not just access to schooling, but full access to the most intellectually challenging program possible. I am also committed to the idea that societies have an obligation to provide the supports necessary so that each student can access as much of the curriculum as possible. I bring to this dissertation the conviction that true inclusion of students with disabilities in all classroom learning and activities should be a significant goal, but also the perspective of a current teacher, with substantial experience in the classroom, which suggests that, in 
practice, there may be times when a student needs to be separated from his or her peers for a short or long time, for the benefit of the student him/herself or the benefit of the group, to a therapeutic environment or one that can provide him or her with virtually fulltime assistance for a disability, at least until sufficient progress can be made so that the student can function in a "regular" school setting.

In describing my experience and point of view, I understand that some of the words and ideas carry cultural baggage, and may contain implicit assumptions about ideas such as "ability" and "individualization" of instruction that may clash with important cultural concepts in other societies. Thus, I have, as much as possible, approached this study with an attitude of skepticism and awareness of the values I may bring, derived through personal background experience and cultural beliefs. In particular, I have a clear awareness that, as an American, and one who has studied special education law and practice in this country, I am likely to view other cultures' educational approaches with a distinctly American bias; I am also likely to bring the biases from my own personal experience, as a student and a parent and as a teacher in a somewhat unusual, progressive and independent school setting, and measure other ways of approaching education against those experiences. I have, therefore, made a conscious effort to counter those values, beliefs and experiences through openness to alternatives in choice, interpretation and analysis of source materials.

\section{Limitations of this Study}

The two chapters that follow this methodology chapter provide a synopsis of general and special education in Sweden and Japan. They were based on comprehensive literature searches and a reading of the relevant literature, published primarily from 1998 to 2013, related to special education in each of the countries, and in the case of Japan, on 
personal communications with Japanese educators and observations in Japanese schools in the cities of Tajimi and Tokyo during my time as a Japan Fulbright Memorial Program Scholar in October 2005. Any time a researcher makes choices about how to search the literature, which articles to read, and among those, which to discuss, which authors to cite, or which issues to emphasize, those choices reflect the researcher's own understandings, cultural limitations and potential personal biases.

The nature of the discussion and analysis reflects primarily the available literature, the literature chosen for careful study and analysis, and the nature of the discourse available in English or English translation for each country. The researcher's ability to interpret the full range of discourse in each country may be affected by bias that may result when certain articles are, or are not, written in or translated into English. Although there is a fair amount of descriptive and analytical writing in English for both Sweden and Japan, it may not, in effect, be a representative sample of all materials written about special education in those countries. It would not be surprising if, for example, works that are self-critical are less likely to be written in English (or translated into English), or published in English language publications. Thus the researcher's language limitations (inability to read either Swedish or Japanese) themselves create a bias. Based on the research described in this dissertation, however, it is likely that both the Swedes and the Japanese are less self-critical, and experience more internal agreement with respect to special education policy and practice, than we do in the United States, and that is reflected in the available literature. In addition, the value of the descriptions and interpretations in this study depends on the quality and accuracy of the translations available, both of which are difficult for this researcher to assess. It is likely, however, 
that, given the large number of both Swedes and Japanese who are bilingual in English, and the peer review process (for those sources in peer reviewed journals or publications of international organizations), that there is not likely to be a systematic bias resulting from inaccurate translation.

The researcher's lack of Swedish and Japanese proficiency affects the study in an additional way - there is far more material available in English relating to special education in Sweden than in Japan, a ratio of perhaps three or four to one. In fact, the RefWorks database created for this dissertation includes 198 articles specifically related to education and special education in Sweden, and about half as many, 94, related to Japan, but the far larger number of articles available regarding Sweden meant that the researcher was more selective before adding articles to the research database for Sweden than for Japan. In addition, there were more articles in which Swedish education was considered in comparison with other countries, particularly articles about education in the European Union, than existed for Japan. In the case of Sweden, a large percent of research articles and commentaries are published in English because there are relatively few Swedish speakers outside Sweden and most educated Swedes read and write English well. A much smaller percent of Japanese writing about education is written or translated into English, although all Japanese study English beginning in junior high school.

Fortunately, the researcher had the opportunity to supplement articles written by Japanese in English (or translated into English) with articles written about Japan by English speakers and by face-to-face communication with Japanese general and special educators. Another significant limitation of this study is the fact that it is a study primarily based on written material, and thus focuses on policy and practice as it is described in the 
literature and legal documents, not how it is, in fact, practiced in classrooms. It has been well documented that practice often differs considerably from stated policy. Tyack and Cuban (Tyack, 1995), for example, noted that teachers, in adopting new policies, tend to modify them so that the changes are not as great as the reformers intended. Arthur Zilversmit (Zilversmit, 1993), based on his study of the adoption of progressive educational practices in three towns, Lake Forest, Waukegan, and Mundelein, Illinois, concluded that, in some of these towns, a traditional educational system was functioning behind the screen of progressive educational rhetoric. It would not be surprising if, for example, despite law that strongly supports and even mandates inclusion, in real schools and classrooms, students were grouped by ability for most instruction because teachers find it easier to prepare lessons for homogeneous groups. Although we should always be aware that what happens in classrooms may be very different from written policy, it does not mean that we should not study policy as cultural and political phenomena. Law and policy in education often set an ideal and that ideal, even if not often reached in practice, tells us much about what people in a nation or community understand and value, and this is an important window into how cultural values affect educational ideas, and how those ideas change culture.

There are also dangers inherent in comparative educational studies. Harold Noah (Noah, 1984) cautioned against ethnocentrism-looking at the world primarily from the view of our own culture, values and practice. That caution has to extend to the choice of study topic, the key words used for searches, the choice of articles to read, the methods of study, and the conclusions we draw. In this case, there is certainly a danger that both policy and rhetoric have been misinterpreted because of cultural misunderstandings. 
Similarly, in analyzing the discourse in articles and commentaries, there is concern about misinterpreting what is written and said because of personal or cultural bias. There is also a danger that either American or personal concerns or distinctions have been applied to the analysis, even if those concerns or distinctions are not important in the societies studied in this dissertation. 


\section{Chapter IV: Sweden}

This chapter tells the story of special education policy and practice in Sweden, and how this policy evolved. The historical presentation is designed to elucidate the way history, culture, policy, politics and law affected the development of special education. In order to make the chronology clear, the section on special education is divided into four periods, the period from World War II to the 1970's, from the 1970's to 1990's, from the 1990's to 2005, and from 2005 to the present. Although these periods seem somewhat arbitrary, they were chosen because the 1970's is the period when, in most places throughout the developed world, there were major changes in special education. In the United States, for example, the first major special education law, the Education for All Handicapped Children Act, was enacted. The 1990's was a period of major political, economic and social change, including an economic recession, and the formation of the European Union. The year 2005 was chosen so that the "recent period" would be about ten years in length. The information contained in this chapter serves as the basis for the analysis and discussion of factors leading to the successes and challenges Sweden has experienced in educating students with disabilities.

\section{Introduction}

Sweden's approach to special education is a clear example of Meijer's (Meijer, 1998) “one-track" system. A relatively small percentage of students, in the 1-2\% range, are formally identified as special education students, and a relatively large fraction of those so identified are served in separate schools or separate classes within mainstream schools. Swedish sources emphasize the concept of a "school for all," in which all 
students, other than the small number of identified special education students, receive the same nationwide curriculum and are expected to meet the same standards. Yet Sweden achieves almost universal literacy within the public compulsory schools, with relatively modest variance in performance. The Swedish education system fully acknowledges and embraces the vast differences in how, and how fast, different students learn, and provides assistance and accommodations, all supported by government funds, to those students having difficulty, literally from the moment of birth through both higher education and a substantial adult education program.

Swedish social policy is strongly influenced by a long-standing political focus on social liberalism, social equity and community, tracing from Sweden's Lutheran heritage. This focus includes valuing all people regardless of social difference or disability, and results in a rich palette of social services provided by the central government. In addition, there is a growing value placed on individual freedom; within the large number of centrally mandated social services, including education, there are multiple options and increasingly local control of delivery methods. Individual characteristics and strengths and weaknesses differ, and are recognized, but are viewed as normal and not "deviant," so no stigma is attached. "Diversity," and the embracing of differences are distinguished from the concept of "deviancy," which labels and treats differences as something negative and abnormal. Viewing learning differences as diversity in Sweden leads to a comprehensive school setting in which educators attempt to make activities more inclusive by decreasing the barriers to exclusion. 


\section{Part I Summary of the General Education System in Sweden}

The goals of universal literacy and social equality are embedded in the Swedish social fiber and educational system. Since Lutheran doctrine that says that Scripture is the final authority on all matters of faith, it was considered basic to the practice of Lutheranism that all members of the state Lutheran Church be literate and able to read Scripture by themselves. Thus, universal education and literacy have traditionally been essential for maintenance of the state religion.

Although education and literacy were prized, Swedish education up until 1970 featured a set of parallel educational experiences for children of different social classes, geared to what would be their ultimate place in the social and occupational hierarchy. As early as 1950, the Minister of Education expressed a goal of minimizing educational gaps among social classes as the main reason for introducing comprehensive schools to replace the many different types of schools serving children during the years of compulsory education. By the 1960's, the social arguments for comprehensive schools became foremost, and there arose a strong political consensus toward creating the comprehensive compulsory school. The success of Sweden's educational reform made it a model for other nations studying school reform within a strategy of social change. The success of the Swedish reform was enhanced by the stability of the general political consensus in Sweden, and the way in which the school was conceived as a major institution of social policy.

Between 1950 and 1962, a number of different configurations and types of schools were studied to try to achieve the goal of creating a more equal educational system. In 1962, the national parliament, the Riksdag, resolved that the comprehensive system should be fully in place by 1972, proceeding in stages, beginning with the first 
three grades and culminating with the integrated upper secondary school. All classes in the compulsory school (age 7-16) were to be heterogeneously grouped, and all students would follow the same program. By 1970, with the transition to 9-year comprehensive schools complete, Sweden ended the long-term practice of maintaining parallel sets of schools at the elementary and secondary levels. A national realexamen was instituted at the end of compulsory school and a studentexamen marked the end of upper secondary school.

As of 1979, Sweden's population was 8.2 million, one of the smaller populations in Western Europe, living on a land area of 450,000 square kilometers, one of the largest, with large differences between its sparsely populated northern region and a more densely populated southern region, which contains its three largest cities, Stockholm, Gothenburg and Malmo. In the preceding century, Sweden had been transformed from a primarily agricultural country to a highly industrialized one, with a significant movement of population from rural areas into cities and towns. Swedish politics had been uniquely stable, with major consensus among political parties on most significant issues. For most of the period from 1932 to 1976, a single party, the Social Democratic Party, was in power, either alone or in coalition with other parties.

Until almost the 1970's, Sweden's population had been largely homogeneous in language, culture and religion ${ }^{11}$. Sweden had a Lutheran State Church for more than 400 years. During the fifteen years from 1965-1979, about one million people of non-Swedish background immigrated, significantly affecting Sweden's former homogeneity. Of these,

\footnotetext{
${ }^{11}$ Although the Swedish population was largely homogeneous until about 1970, it should be noted that there were significant groups of Sami and Finnish speakers, primarily in the northern part of Sweden.
} 
about half were from neighboring countries, mainly Finland and Denmark; the remainder were primarily from southern Europe.

Although there was no legal impediment to the development of private schools under the 1962 Education Act, and even though such schools were entitled to receive state grants on the same basis as public schools, there was very little private school presence in Sweden. Tertiary education was entirely public. In 1979, Sweden had 110 folk high schools for adult students, about half of which were privately run, by religious, trade union or affinity organizations.

In 1979, the integrated secondary school included 22 courses of study and a number of additional specialized courses, ranging from two to four years in length. A two-year secondary school course could qualify a student for university entrance if the student fulfilled requirements in core subjects. Requiring enough general preparatory coursework, so that even those students who chose vocational programs in upper secondary school would meet the minimum requirements for university entrance, delayed differentiation into academic and vocational tracks even later than entrance to high school. Also, in 1979 , there were 275,000 students in adult education, more than the number of students in "regular" secondary schools. Labor market training was entirely statesponsored and free. About a third of each generation pursued some form of tertiary education (hogskola).

Because Swedish law did not regard welfare as assistance to the poor, but as everyone's right, regardless of income, all students in primary and secondary schools were entitled, at no cost, in addition to instruction, to school health services, including regular checkups, dental care, school meals and transportation, teaching materials, and 
recreational activities. Students also received study grants and study loans in addition to free tuition for upper secondary education.

During the period beginning in the early 1980's, the state control of education was weakened in favor of increased power at local and regional levels. This change in Sweden mirrored similar decentralizing changes throughout most of Europe, occurring at about the same time. The economic crisis of the early 1990's brought significant increases in unemployment and immigration. Increased globalization affected both school governance and curriculum, especially through Sweden's entrance into the European Union in 1995. Among the functions of the welfare state, education was one of those most affected by changes in the 1990's. Under the new system, the central government defined more general national objectives, evaluated results and supported quality improvements, but municipalities became responsible for achieving national objectives, and for disbursing funds to reach those goals. "Management by objectives" and "responsibility for results" became the new educational mantras. Terms that have been used to describe this significant change are "devolution," "deregulation," and reliance on “market solutions.” Lundahl (Lundahl, 2005) noted that this revolution in school governance took place with virtually no political controversy, which may explain why education in Sweden has been restructured faster and more radically, in response to international trends, than in other European nations.

Before 1990, it was almost impossible for parents to choose a school other than the one in their catchment (assigned enrollment) area. Since 1990, however, parents have been permitted to choose either private or public schools. The proportion of students in private schools increased from less than one percent to about five percent between 1992 
and 2003. Private schools receive a subsidy of $85 \%$ of the public school per pupil cost. Private schools are required to follow the national curriculum; students are required to take and pass national examinations.

While the changes have occurred with little political controversy, there has been a significant amount of criticism and commentary about the extent and effects of changes in Swedish education over the last 30 years. Nelly Pouragheli (Pouragheli, 2008), for example, suggested that "Sweden is losing its social democratic aspects that once placed it on the world pedestal of social democracies."

As in other countries, an ongoing debate focuses on whether and when during their school careers, students should be divided into academic tracks that ultimately lead to different academic futures and occupational paths. In general, those who see education as a private and economic good have opted for earlier separation, while those who focus on communal and social goods advocate later separation. Typically, the earlier that separation occurs, the greater is the influence of social background on educational outcomes and eventual occupations and incomes.

In 2011, a major new reform of the upper secondary school occurred, resulting in what Alexandersson (Alexandersson, 2011) called a "paradigm shift in Swedish educational policy." One of its goals was to raise the quality and status of vocational training, giving business more direct influence over curriculum, and, at the same time, to raise requirements for the university preparation programs. Upper secondary education was divided into three separate tracks: university preparation, vocational programs, and apprenticeships. This division decreased the possibility that students in vocational programs would graduate prepared to apply for university attendance. 
As part of a Swedish Research Council project, "Upper-Secondary Education as a Market" (2008-2010), Arreman and Holm (Arreman \& Holm, 2011) studied the new role of private schools at the upper secondary level and suggested that privatization has changed the discourse in Swedish education, from social democratic language focusing on the role of education in purposefully inclusive schools, to a language that speaks more about profits than citizenship, equality and pedagogy.

School deregulation is frequently discussed in Swedish media. Media reports focus on such issues as "excessive profit-making," "grade inflation" and "school death," There were also a number of articles about "school death," the closing of both public and private schools because of a shortage of students at schools not chosen by students. Some commentators also suggest that school choice contributed to increased social segregation, and will lead to a decrease in the effects of education on the "public good" and on maintaining democracy and social justice.

\section{Current Educational Structure}

The basic structure of the Swedish education system, prior to the 2011 reforms, adapted from the Swedish National Agency for Education website (The Swedish National Agency for Education (Skolverket), 2011) consisted of:

- Public early childhood education from birth to age 6, provided to all families with parents who are working or in school, or who have children with a disability. There is a small charge, depending on family income and municipality, which is waived for low-income families.

- Preschool education, beginning at age 4, free of charge. Parents may choose whether their children attend preschool, but most children do attend.

- Compulsory education at a comprehensive school, for nine years, ages 7-16, free of charge. Approximately $99 \%$ of all children at these ages attend comprehensive schools. A little more than $1 \%$ attend special schools for students with a variety of disabilities.

- Upper secondary school, 3 years, ages 17-20, free of charge. All young people are guaranteed a place in upper secondary school. More than $90 \%$ of students 
completing compulsory education are eligible for one of the "national study programs," although about twenty percent fail at least one subject on completing compulsory education. Attendance is voluntary, but $97 \%$ of students elect to begin upper secondary school; a small minority drops out before achieving a certificate of graduation. There are seventeen "national study programs," which require a minimum of a "pass" grade in Swedish (or Swedish as a second language), mathematics and English at completion of compulsory education. Students who do not pass may continue their education on the "individual study program," working to fulfill deficits, taking courses from different national study programs, or combining school with work experience. Fifteen of the seventeen programs prepare students primarily for work, in industry, the arts, and health care professions. The remaining two prepare students for further studies. All students take eight core courses, in Swedish (or Swedish as a second language), English, mathematics, natural science, civics, the arts, physical education and health, and religious knowledge. All the national programs provide the courses necessary to qualify for higher education. The number of students leaving upper secondary school without a certificate rose dramatically after 1999, with the introduction of a new grading system. Upper secondary school is typically completed at age 19 or 20 , and secondary vocational programs qualify students for a wide range of employment.

- Adult education programs, free of charge. Each municipality provides education geared for adults at both the comprehensive school and upper secondary school levels. Students who have not completed these levels while at school age may do so through the National Adult Education Program. Adult education programs also provide a vast array of courses responding to the needs of different communities. Large numbers of adults continue to study.

- University education. As of 2008, about $22 \%$ of Swedes had completed a postsecondary education, with somewhat more women than men completing the first university or bachelor's level. This level is higher than virtually any other country except the United States and Canada. Students can attend public universities or more local university colleges. A student can receive a university diploma, generally taking two years or a bachelor's degree, generally taking three years beyond upper secondary school. At the advanced level (postbachelor's), a student can obtain a master's degree taking one or two years. At the doctoral level, students can receive a Licentiate after two years or a Ph.D. after four. There are also professional degrees, taking three to five years after completing upper secondary school. Tuition is free for students from Sweden, countries in the European Union and Switzerland. Until 2011, tuition was free for all students, but since then, students from other countries pay tuition fees. All Swedish higher education students get grant and loan assistance for living expenses; repayment rates depend on income once students begin working. 


\section{Changes in the 2011 Education Act}

The focus of the reforms in the 2011 Swedish Education Act was a desire to

increase scores on international tests such as PISA and TIMSS, to continue the movement

toward greater freedom of choice and to raise the status of teachers. The major reforms as

outlined by Sweden's own website (Sweden. SE, 2012) and the European Commission's

2011 National System Overview on Education Systems in Europe (EU Education,

Audiovisual and Culture Executive Agency, Eurydice, 2011) were:

- Curriculum: Beginning July 1, 2011, new curricula are introduced for compulsory education, high schools, special schools and Sami schools ${ }^{12}$, emphasizing goals for language, science and technology. To assess student progress, new national tests for years 3 (Swedish and mathematics), 6 (Swedish, English and mathematics), and 9 (Swedish, English and mathematics, as well as biology, chemistry, and physics) of compulsory school will be required of all students.

- Grading: Six grades from A to F replace the old system of Pass (G), Pass with Distinction (VG) and Pass with Special Distinction (MVG). Grades are now assigned beginning with grade 6 instead of grade 8 . This is a switch from normreferenced to criterion-referenced grading. Some critics suggest that this change results in grade inflation because the number of high grades is not limited by the normal curve.

- Teacher Certification: Professional certification is required for school and preschool/ kindergarten teachers, beginning December 2013. There are four teacher education programs: preschool, primary education, subject specialist (for secondary school) and vocational education and training. These replace an integrated teaching degree aimed at all levels, with specialization by subject or grade. Teaching degree programs range from three to five and one half years.

- New Structure and Admission Requirements for Upper Secondary School: The existing 17 national programs are replaced by 18 national programs, of which 12 are vocational and 6 are preparatory for higher education. To enter a preparatory program, students must have passed Swedish, English, mathematics,

\footnotetext{
${ }^{12}$ The Sami (Lapp) people have lived in northern portions of Scandinavia and Finland since ancient times. The Sami language belongs to the Finno-Ugric family. Beginning in 1997, the Sami language received official status in parts of Sweden. Sami children may now choose between attending government Sami schools or regular municipal schools where they can also receive instruction in Sami. Sami education in Sweden aims to give Sami children the same national curriculum as other Swedish children, and also to reinforce their own linguistic and cultural background. Information from a Factsheet published by the Swedish Institute, February 1999, accessed at http://www.samenland.nl/lap_sami_si.html.
} 
plus nine additional subjects; to enter a vocational program, students must have passed the same basic requirements, but only five additional subjects. Those who fail to qualify for either program can choose an Introductory Program, which allows them to make up the deficits. Students in the vocational programs can elect to take courses leading to access to higher education in addition to or after completion of their vocational program. Opportunities for apprenticeships were added to the vocational programs. Beginning in July 2012, the new secondary school structure is applied to adult education. Post-secondary vocational education is available to those seeking jobs or job advancement.

\section{Part II Special Education in Sweden}

\section{Historical and Cultural Context}

\section{Summary}

As in most other countries, in the pre-World War II period and during the war, 1935-1945, special education in Sweden was aimed principally at students with physical disabilities or mental retardation. With the advent of ability testing early in the twentieth century, the number of students identified grew rapidly. Research and discourse concentrated on medical factors underlying disability and on technical and teaching aids used to ameliorate any consequent handicap.

From 1945-1965, the Social Democratic Party held power and the Swedish welfare state developed. Special education was provided through a parallel but separate school system. Educators believed that disability was centered in an individual, and that education should therefore be matched with the specific characteristics of that individual. The first Swedish National Curriculum, published during this period, in 1969, specified the contents and organization of a separate special education program.

The period from 1965-1990 marked a period of reliance on educational research and a belief that technical solutions could be used to solve educational problems. The multi-track general education system was replaced by a single comprehensive school system, with "coordinated special education," in which special education teachers taught 
the national curriculum at a slower pace. The 1969 National Curriculum recommended increased integration of students with disabilities into regular classes and, for the first time, acknowledged the contribution of school environment to creating and ameliorating student difficulties. The 1980 National Curriculum further reduced the differences between special and regular education, putting emphasis on prevention of learning problems, and encouraging as much inclusion as possible.

The period beginning in the early 1990's was marked by decentralization and deregulation; local municipalities were given authority over management of funds, including those formerly earmarked for special education. Education policy emphasized the right of all pupils to receive an inclusive education and to get the support they need to meet the requirements of the National Curriculum. However, now individual schools and localities would determine how they should meet this mandate. The role of special educators changed dramatically, from teaching small groups in a segregated setting, to giving guidance to teams of educators responsible for students with the whole range of abilities and disabilities. Researchers questioned whether "special needs" refer to characteristics of individuals or were social constructs relating to how individuals relate to specific school environments.

Some of the elements that led to the Swedish approach to special education are related to the centuries-old traditions of a strong central state, a Swedish governmental structure that separates political decisions from specifically professional concerns, and the belief that social welfare is the business of the government rather than of private, religious and corporate charity. 
In addition to this strong central bureaucracy there were strong grass roots efforts on behalf of disabled people that pressured the government for a place on the political agenda and which wielded significant power in government decision-making. Beginning in the 1950's, parents and disabled persons organized, articulated their position, and pressured the government for better services, framing their requests in terms of citizens' rights. There was an ideological consensus among Swedish citizens that the state, not private charities, should guarantee these rights. Unlike in United States, the role of charity as well as of voluntary organizations has been very limited in Sweden.

\section{World War II to the 1970's}

By the 1960's, all Swedish schools - the nine-year compulsory school, the gymnasium, the continuation school and the vocational school—were open to pupils with disabilities, and special resources were provided to make this integration possible. Special educational teaching was regulated by the Education Act of 1962 (SFS 1962:319), the Care of the Mentally Retarded Act, and other related statutes (Lundstrom, 1969). The Education Act specifically recognized slow learners, retarded maturity, reading and writing disorders, difficulties with mathematics, speech difficulties, difficulties of adjustment (emotionally disturbed), hearing impaired, partially sighted, motorhandicapped, and deaf and blind students as special groups. This legislation stated specifically that even the most severely mentally retarded children are entitled to some form of suitable instruction. Contemporaneous legislation required that every county council must provide for living needs and the care and treatment of students with orthopedic handicaps that would otherwise preclude their attendance in school.

Each teacher at the "junior level" of the compulsory school was given two hours a week to devote to "special auxiliary teaching," with individuals or groups who needed 
additional attention. Pupils who still were unable to keep up with the ordinary curriculum were eligible for "separate or coordinated special teaching," parallel to what was taught in the regular classroom. This was sometimes provided by aides in the regular classroom setting or in "clinics," where teachers worked separately with individual students, who then returned to their general education classrooms. A relatively small percent of students with severe mental or physical handicaps were assigned either to special classes within the school or to a special school setting.

The schools also used "school maturity classes" for pupils who were judged to be immature intellectually, socially or emotionally. These classes typically continued for two years, after which students might return to class with their age peers, go to a lower grade and continue from there, or be judged in need of a class for "slow learners." Accommodations could be made for students with emotional disturbances, or with mild to moderate hearing impairment, by using both "clinic teaching" and, as appropriate, technological aids.

\section{Special Education and Social Welfare}

In 1955, the percent of Sweden's budget allotted to social welfare was about the same as in other industrialized nations. During the 1950's, Sweden, like other similar nations, focused its social service programs on providing a safety net for citizens in economic need based on illness, old age or unemployment. But in the 1960's, a period of high economic prosperity, the role of government was greatly expanded, addressing inequalities pointed out by a variety of interest groups, including the disabled. In the 1970's, the comprehensive school took greater responsibility for disabled persons.

Schools were designed to prevent problems through organizational reforms and flexibility. Building codes were altered to create physical accessibility and employers were required 
to adapt work environments for people with disabilities. The underlying belief was that disabilities would not handicap students in the school or workplace if the environment were constructed to eliminate potential problems. Slogans born in this period were "a school for all" and "a society for all." The concept of the state as the "peoples' home" morphed into the idea of the "strong society," which had the ability and resources to help disadvantaged groups of individuals.

\section{Teacher Roles and Preparation}

The history of special education teacher preparation paralleled the development of special education services in Sweden, with training in the early twentieth century focusing on preparing teachers to work with "defective pupils," but more recently, focusing on the relationship between school environment and school difficulty.

Special education teachers generally perform three kinds of tasks: provision of special care, diagnosis, and teaching. Before the 1960's, "teachers of backward children" were trained primarily as special classroom teachers or as diagnostic specialists, able to provide teaching in "remedial clinics." They were viewed as technical experts in diagnosing and teaching basic skills. In particular, they were responsible for intelligence testing to determine whether students met the strict criteria (IQ less than 85) for admission to special classes for mental retardation. By the 1960's, they were also responsible for diagnostic testing of basic skills in reading, math and spelling. After 1977, teachers training to be special educators no longer had to demonstrate competence in individual diagnosis, but studied "joint problem analysis"- a term that meant working as part of a team to find the right combination of teaching and school environment to enhance students' learning. More recently, teachers were expected to educate their colleagues about appropriate school climate and to provide information about available 
social and welfare support for special education students. By the end of the 1960's, Swedish educators were influenced by research that appeared to show that "backward pupils" who remained in regular classes performed better on cognitive tests than similar students in classes for the retarded.

\section{0's to 1990}

The majority of the special education literature in the 1970's was related to “major disabilities," primarily cognitive disabilities and orthopedic/sensory disabilities. In part, this was because students with major disabilities were often underserved by the educational system prior to the 1970's, but also because less severe disabilities often went unrecognized. Students with these less obvious difficulties typically were those who attended ordinary schools, but often their needs were unmet and they did poorly or dropped out.

In order for the 9-year compulsory school phased in during the 1960's to succeed for the great range and diversity of students who were to be educated, there had to be a strong commitment to individualizing instruction. Educating children with handicapping conditions was seen as a natural extension of this philosophy. Thus, the special comprehensive school for all "disabled" students, those with mental and some physical disabilities, was extended to ten years. This school provided education for students who were considered "trainable," but not educable — those who would not be expected to learn reading and arithmetic, but could be trained in a variety of life tasks, as well as for the most severely afflicted, those considered unable to benefit even from "training." The 10year comprehensive school was followed by compulsory vocational training until age 21 (in some cases 23). There appeared to be a conscious policy that if the educational 
requirement for the typically developed pupils was increased, that the increase for students with cognitive deficits should be even longer.

Lennart Wessman (Wessman, 1970), Inspector of Special Schools, National Board of Education, was among the first to espouse the idea that disability was at least partially environmentally determined. He said, "The need for special education depends greatly on the organization of the regular school system. If the education in the ordinary school was good enough for the handicapped pupil, there was no need of special education" (p. 130).

In 1970, special classes in ordinary schools were replacing separate schools, and the number of special classes was also decreasing, in favor of separate special teaching for students in regular classes. This transition was made much easier within the comprehensive schools, which could more easily fold students with handicapping conditions into the far more diverse academic range, as compared with the narrower ability ranges in the previously tracked schools. Teaching methods within regular schools were becoming more like those that had previously been seen only in special instruction.

The economic slowdown of the 1980's appeared to lead to some retrenchment and decentralization of social welfare policies. The state did not abandon its commitment to social policy, but gave more responsibility to localities, giving localities greater freedom, but often far less money to achieve the goals. While "normalization" and creating environments conducive to minimizing handicap were still explicit goals, progress toward true normalization and integration of handicapped individuals stalled with the economic restrictions. Martin Soder (deVincentis, 1989) observed that localized control 
also sometimes led to geographic inequality, as municipalities made different decisions, and some chose to spend less on education of people with disabilities.

Early detection of handicapping conditions was done through regular government-financed checkups at hospitals and child health centers. Assessment for special educational needs was done by a team of teachers and specialists and always involved parents in both assessment and decision-making. Beyond secondary school, programs were available within colleges and universities, with special colleges for the profoundly deaf and mentally retarded, and available employment assistance and training.

Sweden was one of very few countries that included training in special education in the curriculum for general education teachers. All teachers received ten weeks of study in teaching children with special needs. Special education teachers were recruited from among fully trained teachers with at least three years experience. They received an additional one to three years of training.

In Sweden, the national government regulated preschool education, childcare, disability services, and care for the elderly. Law explicitly stated the right of people with disabilities to lead as normal a life as possible, and gave priority rights for disabled children to places in preschool and after-school centers. County councils were predominately responsible for providing health services, including preventive services and hospital care. County councils were also responsible for habilitation ${ }^{13}$ services for disabled youth. By 1988, these councils moved from organization by specific disability to a more comprehensive approach, in which mobile therapeutic teams offered medical,

${ }^{13}$ Habilitation services are designed to ensure health, safety and welfare, and assist in acquisition, retention and improvement of skills necessary for individuals to participate successfully in their communities. 
educational and psychological support for children with disabilities and their families, depending on need rather than nature of disability.

\section{Normalcy/Normalization}

Americans Mary Jane and Kenneth Clark (Clark \& Clark, 1970) visited institutions and programs for mentally retarded people in three Scandinavian countries, Denmark, Sweden and Norway, and were struck by the prevalence of "normalcy" as the underlying goal of policy and practice in Scandinavia. Programs strived for normalcy, without negating the "retardate's" handicap, by exploiting other abilities so that handicaps became less pronounced. In Sweden, people with cognitive deficits were regarded as having the same rights and obligations as other citizens. An individual's capacity to adjust to the demands of society was regarded as being on a continuum that included both those who were thought of as "normal" and those who were identified as "retarded." The Swedes believed that even the most severely affected could be "normalized" in some respects, and the goal of normalcy should apply even if an individual's handicap is so severe that complete integration in society is unlikely. Individuals were de-institutionalized as much as possible and those who did not work received a pension and "pocket money," so they could make choices about possessions and leisure time. According to the Clarks, the basic principles involved in normalizing lives of individuals with cognitive deficits were:

- People with mental retardation should live and eat in as normal surroundings as possible, with their own room (ideally single occupancy), within a small group, family-like setting as close to their home community as possible.

- They should live in a bisexual world, and sex among residents should not be prohibited. 
- They should work if at all possible, and that work should be in a different environment from living quarters.

- They should be able to choose ways of spending leisure time.

- They should maintain ongoing relationships with parents and relatives and should be able to travel home on weekends if physically able, or be visited regularly by family.

- They should receive all necessary medical care.

In 1983, the Organisation for Economic Cooperation and Development (OECD) published a report on "The Education of the Handicapped Adolescent: The Transition from School to Working Life" (Organisation for Economic Cooperation and Development, 1983). This report stated that for virtually all individuals with disabilities, a regular job is seen to be "the threshold of personal achievement" (p. 12); individuals with disabilities desire to work even when pensions and other financial support are adequate. Swedish policy fully accepted that approach and assumed that the overwhelming majority of those considered "disabled" are able to hold a job. The authors of this report noted that significant medical, technological, and environmental strides made it possible for those with physical and sensory disabilities to hold market employment, and observed that it is important that mildly or moderately retarded students receive training in social skills, citizenship, family living, home management, and basic financial skills, in addition to specific job training and general education.

\section{0's to Approximately 2005}

Beginning in the 1990's, the realities of a world-wide economic slowdown, pressures from Sweden's entry into the European Union, and a growing "neoliberal" political philosophy spreading through Europe and the United States, appeared to be creating pressures in conflict with the goals of full inclusion for students with disabilities in Sweden. Observers noted the radical changes in Swedish schools over a very short 
period and with little policy conflict or public uproar. In 1996, Gary Miron (Miron, 1996) observed, "In four years, this traditional Social Democratic country has promoted school choice, instituted vouchers, and improved the condition of independent schools" (p. 77). Sweden, he said, was one of the last places one would expect to find these educational changes.

Miron further noted that, although education budgets were being cut across the board in the face of the economic downturn, support for children with special needs was especially hard hit. There were large cuts in the numbers of special education and home language teachers and speech therapists, as well as fewer small class groupings for individual attention. He was concerned that these cuts, combined with increasing school choice opportunities, would result in resegregation by ability (or disability), which was counter to longstanding Swedish policy.

Under the decentralized system starting in the 1990's, there were no funds specifically for special education. Municipal authorities decided on allocations for special education needs in the same way they decided on the use of funds for other programs. The amount of funds the municipalities received depended, in part, on the number of special education students, but did not require the local educational agency to spend the money on them.

As a mirror on the changing educational culture, Ingolfur Johannesson and his colleagues (Johannesson, Lindblad, \& Simola, 2002) looked at the changes in educational discourse that accompanied these major educational changes. The language of decentralization, goal steering, accountability, "managerialism," evaluation, choice, competition, privatization, and management by results became central to the new 
discourse of the 1990's. Responsibility for schooling moved from the state to municipalities and schools. The federal role shifted to goal-steering, and schools became responsible for finding ways and managing budgets to meet the goals. They were accountable and responsible for their decisions. Principals became business managers; schools were responsible for self-evaluation and, at the same time, for the performance of their students on national examinations. School choice and competition as well as private school presence increased. The authors noted that the language of equality had virtually disappeared from educational rhetoric.

Bengt Persson (Persson, 1998) observed that, as a result of decentralization, identification and provision of special education services were not consistent across schools or even within a given school, as they had been under the centralized system. Students with very similar diagnoses and educational difficulties might or might not be registered as special education students, and might be in special education classes or in regular education classes, with or without special support. Learning behavior that might be regarded as unusual enough to qualify for special education in one school might be regarded as normal variation in another. With resource allocation decided at the local level, differences in identification for special education from school to school or locality to locality might reflect differences in funding allocation decisions.

\section{Family Support and Subsidies}

But despite all the changes, Sweden held fast to its commitment to social welfare spending, and that interacted with educational provisions for students with disabilities. By the 1990's, the Swedish government had been internationally recognized for its very strong family policy, including daycare, child allowances and government health insurance that were designed, in part, to help identify potential disabilities early in a 
child's life and provide both education and accommodation to minimize the long-term effects of such disabilities. If a child's problem was discovered in the hospital at or soon after birth, a hospital team began planning for the child's rehabilitation and education, and helped the parents understand their child's existing or potential disability. The hospital team connected the family with county rehabilitation centers for infants and young children. The intervention and ongoing contact with the county rehabilitation center continued when the child returned home. The Swedes employed teams of professionals from a variety of disciplines, including medicine, psychology, education and social work, who work with very young children and their families from birth.

Since 1995, all municipalities have been required to provide day care for children aged 1-6, and before- and after-school care for children 6-12, if their parents worked or were in school. Children who needed extra support because of mental, physical or learning disabilities were entitled to a place in childcare whether or not both parents worked. Preschool classes were offered, without charge, to all children aged four and over. Transportation for preschool care for children with disabilities was provided by the government, free of charge. Like curriculum for compulsory school, curriculum for preschool was set by the national government as broad educational and developmental goals to be achieved. Parents of children with disabilities could choose from among available settings for preschool education, and could opt for a mainstream setting even if a separate setting was recommended for their child. Mainstream preschool settings were modified to care for disabled children, with the cost borne by the government. Workers in Swedish childcare were generally far better trained than their counterparts in the United 
States, with mandatory education and training programs, including a university degree, for preschool staff and teachers.

As of 2001 , more than $75 \%$ of Swedish women worked outside the home, and the Swedish government, as a matter of policy, supported parents who both work and raise children. Parents of all children were entitled to a leave of absence from work for up to 450 days, with partial pay, which could be taken any time up to age eight. Fathers and mothers could share in this leave. Parents were also entitled to up to 120 days per year leave at $75 \%$ of pay to take care of ill children, and parents of disabled children were entitled to use that benefit until their children reach age 21. Parents of disabled children were also entitled to an additional ten days of leave per year, with financial compensation, to accompany their children to school or day care. A child benefit allowance was allotted to each child, regardless of the family's income, and additional allowances were provided for families with children with disabilities. Parents with disabled children could stay home to care for a disabled child for a period of up to two years. During that period, the parent remained on leave from his/her job, and retained all social rights and health insurance related to the job.

\section{The Changing Role of Students and Teachers}

In the 1990's, the role of special education schools and teachers had changed considerably. The aim was to make instruction, as far as possible, comparable to that in the regular classroom. Many of the special schools that used to provide education for students with disabilities were transformed into resource centers where specialists provided guidance, assessment, access to materials, and technical aids to teachers in local schools. For students who were unable to complete the regular education curriculum, even with support, the school could arrange a reduced course of study, an extension of 
the time period to complete compulsory school, or alternate work experience with study. Approximately $20 \%$ of all compulsory school students received some kind of special help even though they were not officially identified as special education students.

Beginning in 1990, the course to prepare special education teachers was open not only to experienced teachers, but also to all professionals working in educational settings with children and adults with special needs. The term "special teacher" was changed to "special educator," reflecting a broadening of both tasks and backgrounds of practitioners. Special educators were to become "experts" in adapting curriculum and environments for all pupils; for providing expertise as part of a team working with students with functional and learning difficulties; for analyzing individual difficulties by including social, emotional, environmental and intellectual factors; and for counseling and training other teachers in how to accommodate special education students. Special educators were also trained to plan students' transitions between levels of schooling and between school and work and to find resources for modifying the physical environment for students with physical disabilities.

\section{Cognitive Disabilities}

Starting well before the 1990's, cognitively disabled students were educated in classrooms situated in regular schools rather than in separate buildings. By the 1990's, the curriculum for students with cognitive disabilities followed either a "reading-based" curriculum for students with mild impairment, so called educable mentally retarded (EMR), or a "life skills-based" curriculum with those with greater impairment, called trainable mentally retarded (TMR). Students could also be placed in an "integrated placement" in a general education class, with extra support, following either the reading or life skills curriculum. Approximately $15 \%$ of pupils with a cognitive disability (IQ 
below 70) were mainstreamed. A 1995 law provided that students with cognitive disabilities, including mental retardation, autism and brain damage, could not be enrolled in special education without parental consent. If the parents did not consent, the child would be placed in general education, and would follow the National Curriculum.

The number of Swedish residents who met the criteria for a diagnosis of cognitive disability declined dramatically in the years since World War II. Karl Grunewald (Grunewald, 1997) attributed this to a significant decline in poverty and the effectiveness of early childhood and preventive programs put into place. With no change in diagnostic criteria, students with "mild mental retardation" essentially disappeared as a result of early intervention. School-age youth classified as "mentally retarded" since 1990 tended to include primarily those who were much more seriously impaired.

\section{Upper Secondary, Tertiary and Adult Education}

Most upper secondary schools were comprehensive, with both academic and vocational programs housed within the same building. However, the entrance test to certificate-granting programs and the requirements to complete eight core courses in order to earn a diploma limited the academic participation of some students with disabilities. After graduating from compulsory school, students with significant disabilities still under the age of 21 were entitled to attend physical disability-adapted upper secondary schools, tuition free, including personal care, board, treatment and training.

Among university students, the number with special education needs remained quite small, estimated at about $1 \%$, because most students with academic difficulties tended to end their education at completion of upper secondary school. Swedish higher education institutions each had coordinators for students with disabilities, and universities 
were required to set aside funds to cover support for those students. Available services included technical aids, sign language interpreting, readers, note takers and proofreaders, extra tutoring services, extended time for exams, oral exams, mentors, and modifications to syllabi.

About $50 \%$ of the adult Swedish population of all ages pursued studies at any one time. A state-run system was complemented by municipal programs, giving adults the opportunity to study at both the compulsory and upper secondary school levels, as well as to take job-related courses, or courses following personal interests. This system included adult education for the intellectually handicapped and Swedish language instruction for immigrants. The "folk high school" is a uniquely Scandinavian form, in existence since the mid-19th century. Some courses can prepare students for university study; others are much less formal, and range in length from two to thirty weeks. In addition, many Swedes, including those with disabilities, were involved with labor market training, supported by allowances provided by the National Labor Market Board, or by individual employers.

Of particular interest to students with special needs was the greater flexibility that a "course based" rather than "line based" curriculum could offer. Traditionally, in the upper secondary school, all students in the same "line of study" studied the same subjects at the same pace. The only choice that pupils had was which line of study or special program to choose. Students in a "course based program" took the same eight core subjects, occupying about one-third of their time, but could put together the rest of their program by choosing individual courses. Thus a student could now take more or less than the "normal" three years to complete his or her program. 


\section{Approximately 2005 - Present}

In the years since 2005, research and commentary have centered on whether education of students in Sweden with a variety of "school difficulties" has approached the inclusive ideals of a "school for all." It seems clear from the research that the inclusive ideal has not been fully reached and that, in many cases, inclusive education appears to be decreasing, and "segregation," both within schools and by placing students in special schools and classes, is on the rise. It also appears that most, if not all, of the commentators and researchers are strong advocates of inclusive teaching for almost all students and that there is an ongoing tension between those researchers and the teachers and school administrators directly responsible for teaching and placement decisions. There appear to be significant impediments to increasing inclusive practices, resulting first from economic constraints, and second, from the relatively new competitive environment, which allows parents to choose schools based, in part, on published reports of schools' achievement scores.

\section{Performance at End of Compulsory School/Longitudinal Studies of Performance}

Despite the apparent success of the Swedish school system and programs for

assisting students who need extra help, there is still evidence that students who receive extra help, with or without a formal diagnosis, generally do not catch up with students who never receive such help.

Ulla Ek, et al. (Ek, Westerlund, Holmberg, \& Fernell, 2011) studied final grades at end of compulsory school (age 16), comparing those who had been diagnosed with ADHD or behavior and learning problems (BLP) with those who had neither diagnosis. Final grades of students in the ADHD and BLP groups were significantly lower than grades in the comparison group. Ninety-two percent of those in the comparison group 
received qualification for study in upper secondary school, while $68 \%$ of the ADHD and $72 \%$ of the BLP group did so.

Giota, Lundborg and Emanuelsson (Giota, Lundborg, \& Emanuelsson, 2009) analyzed data from a large longitudinal study, "Evaluation Through Follow-up," of approximately 35,000 children born in the years 1972, 1977, 1982 and 1987. About 40\% of each cohort received special assistance during some part of their education through 9th grade. They divided the students into groups, depending on whether they received services and, if so, the kind and length of services they received. Students who received any support nevertheless were less likely to receive passing grades on 9th grade tests than students who never received support. Students who received support in early school years scored lower than students who received help later; students who received help over longer periods scored less well than those who received help for shorter periods, Despite special education support, even given early in a student's education, special needs appeared to become "permanent," and strongly affected skill attainment at the end of compulsory school.

In 2008, Bengt Persson (Persson, 2008) noted that students with disabilities generally meet academic requirements at the end of elementary school as well as students without disabilities, yet about two-thirds of them took individual, rather than national, programs at the secondary level, causing them not to complete requirements for any form of higher education.

\section{Reading/Dyslexic Difficulties}

One of the consequences of the relatively informal way of dealing with minor disabilities, as well as of a cultural approach to education that is willing to set different time patterns for different students appears to be that school difficulties, and difficulties 
in learning to read, in particular, appear to be recognized later in students' careers than they are in the United States. Lise Roll-Pettersson and Eva Heimdahl Mattson (Mattson \& Roll-Pettersson, 2007; Roll-Pettersson \& Mattson, 2007) reported that students’ difficulties in reading and writing were often not acknowledged by schools until late elementary school (grades 4 or 5), based on the belief that students develop at different rates and that problems would be alleviated with maturity. However, a majority of parents said that they were concerned as early as preschool that their children might have school difficulties, and alerted the schools about their concern in early grades.

\section{Goals to Attain and Goals to Aim For}

For each curriculum goal, Swedes list two kinds of goals for instruction: "goals to attain," generally basic concrete and measurable skills required for a grade of "pass," and "goals to aim for," higher level skills required for grades of "pass with distinction" and "pass with special distinction." Despite the official rhetoric of a school and curriculum for all, Kjellin, et al. (Kjellin \& Wennerström, 2006; Kjellin \& Granlund, 2006; Kjellin, 2007) observed that many teachers aimed lessons for students with disabilities at meeting the required "goals to attain," and excluded students who had not mastered them from classroom dialogue aimed at higher level understanding. They noted that, even though some students with reading difficulties might be able to reach "goals to aim for," it is unlikely they would be able to reach these goals if they are exposed only to activities aimed at teaching basic skills. Interestingly, students often appeared to be more engaged in activities designed to teach more complex skills. The authors cited an ongoing dilemma for educators of students with special needs: in order to "pass," students need to master basic skills, and they tend to learn them more slowly and need more support than other students. However, if these students spend most of their school time working on 
basic skills, they miss out on stimulation of higher level thinking, and thus on the opportunity to develop the skills they need for both higher education and full participation in society.

\section{Education of Deaf/Hearing Impaired Students}

The pattern of inclusion and emphasis on normalization is broken for deaf or hearing-impaired students whose first language is sign language, apparently out of concern for honoring "deaf culture." Education for such students is provided in separate schools under the National Agency for Special Schools for the Deaf and Hard of Hearing (SPM). Sangeeta Bagga-Gupta (Bagga-Gupta, 2007) looked at segregated schools for the deaf as an illustration of the way Sweden deals with the unique situation of individuals who are given both cultural minority status (as members of a "linguistic minority") and handicap status, and the contradiction it poses with the values underlying the inclusive "school for all." Even in periods when oral communication was strongly emphasized, Swedish Sign Language (SSL) and Swedish were both recognized as the official languages of instruction. There is some evidence that this is about to change because currently $80-90 \%$ of Swedish students who are born deaf receive cochlear implants shortly after birth, completely changing deaf education. However, some "deaf activists" view this approach as an instance of modern society's continued oppression of the deaf through new medical-technical means. Gupta asked what views of human difference, diversity and normalcy underlie the decision to give virtually all deaf children cochlear implants. Will there be children for whom cochlear implants will not be completely successful, and should there continue to be segregated schools for the deaf for such students? 
In two articles, Rydberg et al. (Rydberg, Gellerstedt, \& Danermark, 2009; Rydberg, Gellerstedt, \& Danermark, 2010) looked at the status of educational attainment and employment for deaf people in Sweden. Despite a legal requirement that education at schools for the deaf should be equal in content to education provided for hearing students, deaf people at all ages had completed less schooling and fewer had attained higher education than non-deaf people. Although, on average, younger deaf people who had completed their schooling had more years of schooling than older ones, the differences in educational attainment between hearing and deaf people have increased rather than decreased because average school completion rates increased faster for non-deaf students than for deaf ones. Rydberg et al. also observed that employment rates for deaf people were significantly lower than for hearing people in the same age cohort.

\section{Political and Legal Factors}

\section{Critical Appraisals of the Changes in Swedish Special Education}

Several commentators have been critical of the changes in Sweden's educational policies affecting special education since the 1990's, and have suggested that these changes are in conflict with the long-espoused Swedish social and equalizing goals of education. It is interesting that no one appears to question the underlying principles or the role of government in equalizing opportunity. For example, Anne-Lise Arnesen and Lisbeth Lundahl (Arnesen \& Lundahl, 2006) asserted that the apparent rationale for education in Sweden has changed from emphasizing collective values and community to focusing on individual academic progress, choice in education, and the rights of students and their families. They observed an increased tendency to look at special needs students as students with individual problems to be solved through individual treatment, instead of looking at the system to see why it did not meet their needs and how it could be adapted 
to do so. Antikainen (Antikainen, 2006) asserted that, despite the accomplishments of free comprehensive schools for all, education has not been the "great equalizer" it was supposed to be. Instead, he observed, education continued to be a mechanism of social reproduction. He suggested that the more schools track students and the greater the range of student performance, the more significant are the effects of socioeconomic background on achievement. Under a broad interpretation of equality in education, schools intervene to ameliorate factors causing differences, particularly cultural, linguistic and social differences and disabilities.

Jane Brodin and Peg Lindstrand (Brodin \& Lindstrand, 2007) focused on the failure to reach fully the concept of a school for all, and the application of the "Normalization Principle" for disabled students. Despite many positive strides, they stated that there still remains much work if the goal is to include all students fully and naturally in the social community. The authors observed that an ongoing obstacle to full social inclusion is the concept of "acceptable" and "unacceptable" school behaviors, which may differ from place to place and situation to situation. Special support, they said, should be regarded as a right of all children, with or without specific diagnosis, while, under current practice, diagnosis is sometimes required to access special support. The people who decide what is "deviant," and who needs special education, wield substantial power over students' lives. Also, they noted that that economic factors disproportionately affect students in need of additional help. As funding decreases, class size increases, and with it the ability to attend to diversity within a class. This, in turn, appears to result in resegregation of classrooms, with students increasingly learning in separate groups. 


\section{Identification Issues}

Neither Swedish school law nor regulations mandate how students are to be identified for special education. Thus the process for identifying students to receive special services rests at the municipal and school levels. Isaksson et al. (Isaksson, Lindqvist, \& Bergstrom, 2010) believed that the most crucial part of special education is assessment, because it is there that some students are judged as "different," and selected to receive an education different from that received by "normal" children, identifying some learners as "normal" and others as "less than normal." Isaksson looked at three possible identification models: medical (related to illness); social (related to personal adjustment and behavior); and pedagogical (related to success in meeting knowledge goals). They noted that the medical model is still the dominant way to categorize pupils, and asserted that this model puts the "blame" for a student's difficulties on the student, rather than on the school, or the interaction between the school and the child. Both the pedagogical and medical models tend to use some kind of objective test; the social model uses normative and non-objective judgments of behavior. Since school officials were often the initial identifiers of students, a school or individual class becomes the framework for what is regarded as "normal" or "deviant," and the boundaries of the "normal" range may differ greatly school to school and even class to class. Despite the widespread use of "objective tests," special educational needs tended to be constructed relative to a school's resources, especially when diagnoses generated increased resources for schools.

\section{Use of IEPs}

IEPs were first used in Sweden in the 1980's (Isaksson, Lindqvist, \& Bergström, 2007), but were not required until 1995. IEPs were to be developed with the cooperation 
of the school, the student and his or her parents. Despite the requirement that parents be involved, many IEPs were established without parental involvement, and a substantial number of parents were not even aware of the existence of IEPs for their children. IEPs tended to relate educational difficulties to deficits in the individual rather than to the effects of school organization and teaching environment, and measures to ameliorate such difficulties tended to involve special skills training and attempts to "fit the child to the curriculum," rather than changing the school environment to make the curriculum more accessible.

\section{Summary}

Part II of this chapter describes special education policy and practice in Sweden, focusing on the influence of Sweden's social welfare model and its related "school for all," both of which are central to Swedish culture, and how they influence the education of students who learn differently from the majority. It also discusses the more recent impact of "neoliberal philosophy" and membership in the European Union, which decentralized the school system and added a fair amount of choice, but which maintained Sweden's commitment to a strong central government, whose role is to assure full access to educational and occupational opportunities, as well as a wide selection of other benefits. On a theoretical level, Sweden continues to embrace inclusion for all but a very few most severely disabled students, and generally includes, and exposes to the full national curriculum, close to $99 \%$ of the school-aged population. This reflects a view that observed disability results from both personal and environmental factors, and that both need to be addressed to limit the effects of disability and create the greatest opportunities for all students. Still, there is ongoing disagreement among researchers, policymakers and those with their "boots on the ground"- - teachers, parents and, in many cases, the 
students themselves. Many observers see that the medical/psychological model persists and students are still diagnosed and categorized by disability, rather than by functional needs. Several commentators have expressed great concern that the major policy and financial changes in the last 20 years have moved Sweden away from the democratic and inclusive ideals that apparently continue to be central to the Swedish culture and an overwhelming national consensus. 


\section{Chapter V: Japan}

This chapter, paralleling the preceding chapter on Sweden, focuses on special education policy and practice in Japan and how this policy evolved. It also contains a summary of general education structure and policy to frame the discussion of special education, and is similarly organized chronologically, using the same time periods as a framework to shed light on the effects of history, culture, policy, politics and law on the development of special education. As in the chapter on Sweden, the information contained in this chapter is used to inform the analysis and discussion of factors leading to the successes and challenges Japan has experienced in educating students with disabilities.

\section{Introduction}

Starting with the period of American occupation immediately after World War II, Japan has often adopted or adapted ideas from the United States rather than from other countries. Much of the Japanese special education system prior to the implementation of the new Special Needs Education Law of 2007 was based on the advice given by a United States commission shortly after World War II to set up special schools to serve handicapped students. Prior to World War II, most students with significant disabilities were cared for at home and did not have an opportunity to go to school, so providing education for students with such disabilities, in the 1940's, could be considered a major step forward.

A medical model, in which doctors made a diagnosis of disability and labeled it by category (such as deafness or blindness) made sense within the Japanese culture, 
because students with a diagnosable medical problem could comfortably be treated differently from other students. And, Japanese culture was served by having virtually all other students taught together in regular classes, with few services and very little special attention for those who learned differently. In the past 20 years, some Japanese educators have become aware that there are non-diagnosed students in regular classes who fall seriously behind, and that these students have not been fully served. Since 2007, Japan has adopted definitions of some formerly unrecognized disabilities, such as attention deficit disorder and learning disability that are very similar to those used in the United States since the 1970's, but Japanese schools have been very slow to adopt many of the ideas in America's special education approach and use them in the classroom, probably because they clash with some basic cultural precepts, such as the absence of a concept of "ability" and the prominence of communal over individual values.

Japanese cultural tradition holds that, with rare exceptions, all people are born with equal capacities to achieve. There are thus no perceived differences in "ability," only differences in mastery level at a given time. In fact, the concept of "ability" and the range of ability tests that are used in many other developed countries have had little meaning to the Japanese, at least until the last few years. Differences in performance are generally attributed to the degree and quality of individual effort, rather than to differences intrinsic to the individual. Because students are presumed to have similar ability, Japanese traditionally have viewed special attention as discriminatory (Barker, 1996; Holloway, 1988; Murakami \& Meyer, 2010a).

Grouping by ability or performance, whether for struggling or outstanding students, violates students' rights to an equal education and would be harmful to slower 
learners, who would not get the benefit of the full national curriculum. Japanese have defined equality in terms of the "inputs" of education —all students receive the same curriculum, materials and instruction, rather than in terms of its "outputs"- the results that students achieve. This equality of educational inputs breaks down at grade 10, entrance to secondary school, when placement in ranked and achievement-segregated high school programs depends on students' performance on competitive examinations. Japanese culture has emphasized the importance of the community over the primacy of individual needs. Individuals achieve their identity by their place in the communities to which they belong — their family, their school class, and later the company they work for. Japanese education, as a very conscious transmitter of cultural values, is designed to foster that sense of community, both implicitly and explicitly.

This chapter summarizes special education policy and practice in Japan, focusing on the cultural, political and legal underpinnings that are the foundation of Japanese special education. However, in order to understand the context in which special education efforts have developed, the first part of the chapter reviews the structure and characteristics of the general education system. In Japan, where all but a very small percent of students, those with major, low incidence, primarily motor or cognitive difficulties, attend general education schools, with little specific support for students with disabilities, the general education system is often the only "special education" offered. The second part of the chapter focuses on the special education system, and looks at historical, cultural, policy and legal factors that underlie the way it was constructed and the ways in which it succeeds in educating most students at a relatively high level. To tell 
the story of how these factors affected educational policy, the information in each section is generally presented chronologically.

\section{Part I Summary of the General Education System in Japan}

After World War II, during the allied occupation, the new post-World War II education system was modeled after what was then current in the United States. In 1946, a delegation of education experts, headed by George Stoddard, New York State Secretary of Education, was sent by the United States Office of Education to establish, essentially from scratch, a democratic education system. These officials brought with them thencurrent progressive ideas, such as those of John Dewey, and imposed many of those ideas on what had been a very conservative society and educational system. Since this massive cultural transfer, many elements of that era's progressivism persist. However, some have been rejected in the years since the occupation and still others have been modified in order to fit with the underlying Japanese culture. For example, the American advisors encouraged the establishment of comprehensive high schools following the United States model. Only a few prefectures (comparable to states in the United States) created these high schools, but by 1963 all high schools were academically stratified, with admission based on entrance examinations. The 1946 Report of the United States Education Mission to Japan established the 9-year compulsory education system, abolished "ethical code" education (regarded as nationalistic and militaristic), initiated coeducation, established teacher training at the university level, and supported equal access to higher education. The United States attempted to transform the very centralized pre-war system into one that was more decentralized, as in the American model, but the Japanese system became recentralized after restoration of full national sovereignty in 1952. 


\section{Current Educational Structure}

Throughout Japan, education is compulsory in grades 1-9. Unlike the United States, where localities differ with respect to structural groupings (e.g., middle schools, K-8 schools, etc.), the structure described as 6-3-3-4 is uniform throughout the country. First through sixth grades (ages 6-12) are served in elementary schools. Seventh through ninth grades (age 12-15) are in separate lower secondary (or junior high) schools. There are private, but very few public, kindergartens (for students age 3-5), daycare centers and centers for early childhood education and care. Education before age 6 is not compulsory, but has become much more popular in the last 20 years, as more women work outside the home. Almost all elementary schools are public; there are very few private schools at the grades 1-9 compulsory level. At the high school level, private schools are a significant presence, accounting for up to $29 \%$ of students.

Students, no matter how poorly they are doing, never repeat a grade within compulsory education. At the end of the compulsory period, during grade 9 , students take academic tests to qualify them for high school entrance. A student's score on the grade 9 tests determines the schools the student is eligible to attend. Ninety-nine percent of all students attend upper secondary school, grades 10-12, including both academic and technical schools, with virtually all who enter graduating. About $50 \%$ of students completing upper secondary school continue to higher education, at either nationally-run or private universities or junior colleges, although the junior college presence is relatively small. In Japan, national universities are the most prestigious; there is great competition among high school students to qualify, with about $27 \%$ of those applying to university accepted at public universities. Virtually all students beginning four-year bachelor's degree programs, about $25 \%$ of the age peer group, complete them. 
Since the 1960's, Japan has been an "educational credential society," (Barnes, 1999), in which the high school and university a student attends determines, in large part, his or her occupational opportunities as well as social status, so there is significant stress during the process of trying to get into the "right" upper secondary school and university. The stress has been termed "examination hell," and has been described as overwhelming for many students and their families. There are, Barnes noted, fewer "second chances" in Japan than in the United States. Lucien Ellington (Ellington, 2005a) noted that universities are often thought of by Japanese themselves as the weakest component in the Japanese system. Outside of science and engineering, Japanese students often indicate that there are few academic demands once they gain admission. Further, until recently, graduate education and research were much less well developed than in the United States and Europe.

Administration of the educational system is highly centralized. The Ministry of Education, Culture, Sports, Science and Technology (MEXT) sets the courses of study for grade 1-9 compulsory schools, and pays for the cost of textbooks and half the teachers' salaries in those schools. MEXT also organizes ongoing training for teachers, subsidizes the cost of building schools, and runs the national universities. Japanese texts for primary and secondary school are privately produced, but must be approved by MEXT.

Textbooks in Japan tend to be much shorter than in the United States, cover fewer topics, and stick very closely to the National Curriculum. Curriculum, but not pedagogy, is prescribed; teachers and schools have leeway as to how they present the required lessons.

Compulsory school is free in Japan, but until 2010, upper secondary school was not. In 2005, attendance at upper secondary school cost approximately $¥ 9500$ a month, on 
the order of $\$ 100$, depending on the exchange rate, but there was still virtually $100 \%$ attendance, evidence of families' strong commitment to education. Since 2010, tuition has been free at public high schools. National and prefectural universities cost about $\$ 10,000$ per academic year, and tuition at private universities can exceed $\$ 15,000$ a year (depending on exchange rates).

In Japan, teaching is a prestigious vocation with lifelong employment, a very low turnover rate and almost no teacher shortages. Teachers in special education classes or schools are paid an additional stipend of 6-8\% above their regular teacher salaries, although only one-third of such teachers have a special education license. Only 7-8\% of Japanese teachers hold master's degrees; there is no extra stipend for additional education, only for years of service.

$J u k u$ (or "crammies") form a "shadow" school system of private schools, paid for by parents, for students in compulsory and upper secondary schools, which operate entirely outside the Japanese public schools. There are a variety of juku providing remedial assistance, test preparation, academic enrichment and/or enrichment in the arts. They are competitive businesses, dependent on meeting the perceived needs of paying parents. As a result, they keep class sizes small, carefully hire and monitor teachers, and prescribe exactly what and how they will teach. Approximately $40 \%$ of third graders and $60 \%$ of sixth graders attend $j u k u$, with much higher percentages at the junior and senior high levels.

Japan's society is far more ethnically and racially homogeneous than the United States $^{14}$, and until recently, when it has begun changing slowly, Japan has not been faced

\footnotetext{
${ }^{14}$ See data from http://www.worldpopulationstatistics.com/japan-population-2013/ cited above.
} As a comparison, the corresponding data for the United States, 
with significant numbers of students who do not speak Japanese as a first language. Nevertheless, there are some minority students, who have traditionally been at both educational and economic disadvantage in Japan, including Buraku (descendants of outcast communities of the feudal era), Ainu (an indigenous group that originally spoke the Ainu language and lived in Hokkaidō, the Kuril Islands, and much of Sakhalin, but who now speak Japanese), ethnic minority Koreans, and other foreign students.

\section{Current Issues-Recent and Proposed Reforms}

Although there is no evidence that education is a significant political issue in Japan, there is certainly some ongoing conflict between advocates of what might loosely be called conservative and progressive points of view on the directions that Japanese education should take. The "conservative" viewpoint asserts that Japanese education does not currently champion traditional Japanese values and cultivate patriotism and respect for Japanese history and traditional culture. This viewpoint advocates increased specific moral education, increased focus on supporting an educational elite during the compulsory school years, and greater emphasis on special services for gifted students and increasing the number of highly skilled graduates, to enhance the growth of Japanese industry. The "progressive" point of view criticizes the conservatives as supporting restoration of a neo-nationalist state and maintaining superiority in the global economy by nurturing educational elites at an even younger age. The progressives suggest that conservative proposals to address individual differences are directed primarily toward the

http://www.worldpopulationstatistics.com/us-population-2013/, show that the largest ethnic group is those that identify as white, which makes up about $72 \%$ of the population. The next largest ethnic population is the black American group, which makes up about $13 \%$ of the population. The Asian population makes up about $5 \%$ of the population and is thought to be the fastest growing ethnicity in the entire country. The rest of the population is made up of Hispanics and Latinos, American Indians, Alaskan Natives, Native Hawaiian, Pacific Islanders, and those that identify as multiracial. 
highest achievers, and that they ignore the needs of children who do not perform as well. Other progressives view the more progressive approach as a move to a more relaxed education and a focus on "zest for learning," a reaction to the tensions of "examination hell" and the observation that Japanese students may appear not to be as analytical and creative as students elsewhere in the world.

There have been several rounds of reforms designed to address some of the issues raised by critics coming from both political and educational points of view. A 1995 school reform attempted to introduce more creativity, diversity and flexibility into the Japanese education system. The Central Council for Education proposed increasing "zest for learning" and giving children utori, "room to grow." At the upper secondary level, a greater variety of high school curriculum options significantly decreased the high school dropout rate, and allowed students who are less inclined to academic study to complete high school by earning required credits at the same time that they are working at various jobs.

In 2002, Japanese officials reduced school hours by eliminating Saturday classes and also decreased the number of hours devoted to academic studies (although, in practice, many children now use that extra "free time" for instruction at juku). They introduced a course in "integrated studies," and allowed local freedom to choose topics covered. Whether or not there was any direct relationship, shortly after introducing these reforms, national ranking on international tests such as TIMMS (Trends in International Mathematics and Science Study) and PISA (OECD Programme for International Student Assessment) dropped, at least as compared with previous first or second place levels. 
In December 2006, Japan enacted a new Basic Act on Education (Ministry of Education, Culture, Sports, Science and Technology (MEXT), 2006). This was followed in July 2008 by a detailed Basic Plan for the Promotion of Education (Ministry of Education, Culture, Sports, Science and Technology (MEXT), 2008). Together, these actions put forward a vision for the next ten years, as well as a detailed plan of measures that were to be implemented over the five years from 2008 through 2013. These plans sought to address both kinds of concerns - those that focused on a need to increase creativity and "zest for learning," and those that focused on a need to reinvigorate academic rigor and the teaching of moral behavior and traditional values.

Within the Basic Plan, MEXT prioritized the following elements, and the 2006 Basic Act on Education contained a provision for assistance for education of people with disabilities:

- Commitment to new Courses of Study, aimed at increasing "solid academic abilities," including both fundamental skills and logical-critical thinking, as well as "zest for learning" by the end of fiscal 2008, with full-scale adoption for elementary schools in fiscal 2011 and for junior high.

- Introduction and administration of a new National Assessment of Academic Abilities for compulsory school to be used to improve high school level instruction.

- Promotion of moral education, education relating to Japanese tradition and culture; increased hands-on activities, including nature programs, volunteer activities, cultural activities and vocational experiences.

- An increase in physical education time to improve physical strength, health and athletic ability; regular assessments of "physical strength."

- Improving measures to address delinquency, bullying, violence and school refusal.

- Encouraging early childhood education.

- Improving teaching quality and creating an environment in which teachers can work one-on-one with students.

- Promoting education for children with special needs, including creation of "individual teaching plans." 
- Encouraging community-wide efforts to improve education, including assistance to families, forming partnerships with community groups.

- Using school buildings for an "After-School Plan for Children," to establish a safe environment for hands-on learning activities after school and on weekends.

- Promoting career and vocational education; increasing opportunities for lifelong learning and return to education for adults.

- Promoting university education, research at the university level, and increases in foreign students.

- Increasing safety in schools through improved facilities.

- Promoting private education through financial and management assistance.

- Decreasing financial barriers to learning, through assistance in paying for private schools and college, giving serious consideration of making preschool education free, and supporting scholarship programs and Private Education Institution Aid.

In summary, starting with a post-World War II system created by and based on the entirely new educational ideas brought to Japan by the American Occupation, the Japanese have, over the last 70 years, created a highly successful educational system that is consistent with elements of Japanese culture. Despite its overall apparent success, there has been continuing criticism and pressure for change from two elements, seemingly in opposition to each other, but both of which have been able to influence recent law and changes in practice. One sees the Japanese system as overly pressured and producing students who are competent, but not creative problem solvers, and advocates increasing opportunities for increased creativity and "zest for learning," and decreased test pressure. The other is more concerned by what they see as falling test scores and a decrease in moral education. They advocate more frequent testing, especially in the pre-high school years, more emphasis and time on academics, and a commitment to education in the traditional arts and sports. 


\section{Part II Special Education in Japan}

As with the chapter on Sweden, the description of the development of special education in Japan is divided into four sections: World War II to the 1970's, 1970's to 1990's, 1990's to 2005, and 2005 to the present.

\section{Historical and Cultural Context}

Because Japanese culture has traditionally emphasized the family's role in supporting its members, there were few public services for individuals with disabilities prior to World War II. Most children with disabilities were cared for by their families and communities and not considered eligible for schooling.

In the nineteenth century, when Japan first began to interact with the outside world, there were two competing perspectives on disabilities: the older one, that those with disabilities were useless and to be excluded from education; and the newer one, that some individuals with disabilities could become productive through active intervention. Education for those who were deaf or blind (the so-called "traditional disabilities") developed first, as it did in many other countries, because educators believed that those with deafness or blindness could be productive despite their disability.

\section{World War II to the 1970's}

The 1946 Report of the United States Education Mission to Japan, which set up the modern Japanese educational system, also addressed special education, recommending a segregated system of special education to serve physically handicapped, mentally retarded, blind and deaf students. This extension of education to students with disabilities, along with creation of a separate set of schools mirrored practice in the United States at that time and represented a major increase in the number of students with disabilities who were to be served by schooling. During the American occupation, special 
schools were created, separated by type of disability: deafness, blindness, mental retardation and physical disabilities. Compulsory education was extended to deaf and blind students in 1956, but not to students with physical and cognitive disabilities until 1979.

In the economic boom period from 1950 through the 1970's, many more residential schools were built for students with severe disabilities, but this building boom continued to leave them institutionalized and segregated. Some change was observed in the 1990 's, as more students with disabilities enrolled in regular schools as "exceptions," leading to more special classes and resource rooms in mainstream schools. The first recorded recognition of the idea of inclusion appeared in a 1969 report on special education, which suggested that "extreme segregation" of students with disabilities should be avoided and that there should be more opportunities for interaction with students without disabilities.

Laws in the 1940's and 1950's had provided for compulsory education for "handicapped" students and for supporting institutions for their education. But, while there were funds for buildings, books and transportation, there were none for staff, staff training or curriculum development. In 1962, the Ministry of Education published a first curriculum and some textbooks for "mentally retarded" students that paralleled the regular education curriculum. It also included vocational training geared to local employment needs and evaluation that focused on full use of abilities, social adaptation, and ability to fulfill a role in society. However, no services were available for children post-school age, although there was some employment assistance. Because many 
Japanese families were uncomfortable with separating children from their families and communities, many students remained in their communities and did not attend school.

Teachers received both regular and special education licenses through the central government. The special education license required either an inservice course for teachers with regular licenses or completion of a two- to four-year course at one of seven universities that offered special education programs.

\section{0s to 1990's}

In the period from the 1970's through the 1990's - a period of significant change in special education policy elsewhere in the developed world, ${ }^{15}$ changes were slow to come to Japan, and virtually all special education remained segregated in special schools and classes.

In 1978, before the full enforcement of compulsory education for handicapped students, a Special Education Study and Research Conference reported to the Ministry of Education on special education for students with "mild handicaps." They recommended the use of resource rooms or itinerant teachers to support students with mild physical handicaps, including physical weakness, partial hearing or sight, emotional disturbance or speech impairment. However, as Yoshihisa Abe (Abe, 1998) reported in 1998, their services still reached a very small number of Japanese students, particularly beyond the elementary level. Abe called the education of students with relatively mild handicaps the "foremost special education issue in Japan." While some educators recognized the

\footnotetext{
15 The Education for All Handicapped Children Act (Public Law 94-142), the forerunner of the current Individuals with Disabilities Education Act, was enacted by the United States Congress in 1975, ushering in the "modern age" of special education in the United States.
} 
existence of attention disorders and learning disabilities, students with learning disabilities were not identified for special education in Japan. ${ }^{16}$

Those students who had apparent needs for extra help, but who were not formally identified, were usually taught in general education classrooms. In many cases, the teacher attempted to give them special attention within those classrooms, but help was often limited by large class size (typically about 40 students) and limited teacher training in special education. For specific subjects, such as Japanese language, there were sometimes special facilities or assistants, but large student load and parental resistance to labeling or singling out children for special help meant that the majority of students who needed special help were not getting it.

Limited preschool education of children with disabilities started shortly before World War II (1938) in the Aiku Research Institute for Maternal and Child Welfare. Although closed during the war, it reopened in 1949. The number of nursery classes for children with disabilities expanded, especially during the 1960's and 1970's. Through this period, behavior modification techniques were a principal approach to educating young children with disabilities. In the 1980's and 1990's, as the general population of children decreased, mainstream nursery schools and kindergartens were more willing to accept children with a variety of handicapping conditions.

There were a few voices for increased inclusion of students with disabilities, who spoke out against the law that made special schools compulsory for such students, including work by M. Shinohara, reported in (Suzuki, 2008). Shinohara was unusual among scholars for opposing the 1979 law that made it compulsory for students with

\footnotetext{
${ }^{16}$ For comparison, students identified as having "learning disabilities" represent approximately half of all students identified as eligible for special education services in the United States.
} 
intellectual or physical disabilities to attend special schools, while many professionals in the disability field applauded this law because it meant that students who were formerly excluded from school altogether would now have some access to education. However, the law also created a legal basis for excluding students with disabilities from regular education.

During the 1970's and 1980's "scientific special education" and "the principle of ensurement of development" dominated discourse about education of persons with disabilities. This principle emphasized the idea that disabled children had the "right to develop," and took a "scientific" approach to increasing and assisting that development, but took institutionalization for granted as the best or only way to achieve that objective.

\section{0's to 2005}

During the 1990's, discourse regarding special education broadened with an increased awareness of students who had academic difficulties, but who did not have mental retardation or physical disabilities. Although a few educators who had read literature from the United States and other Western countries were familiar with the concept of "learning disabilities," by the mid-1990's the concept remained unrecognized in much of Japanese educational practice. In 1995, the Cooperators Conference for Research and Study of Students with Learning Disabilities adopted a definition of learning disability that parallels the definition used in the United States, by defining it as an underlying "central nervous system dysfunction," which is not caused by, but can be coincident with, other conditions that interfere with learning, and is manifested by a difference between ability and achievement. Despite the report of this conference, it appears that, other than the adoption of a definition, and greater public awareness of the words "learning disability (LD)" and "attention deficit disorder (ADD)," there was no 
substantial change in practice. The issue was once again raised in the 2001 Final Report on Future Direction for Special Education in the 21st Century. The name "special education" was changed to "special support education," to include not only those students with disabilities, but those who were experiencing difficulties in learning, but were not "disabled."

As of 1992, about half of students identified as needing special education during the compulsory period from grades 1-9 went to one of 980 separate schools. The other half were served in self-contained special education classes within some public elementary and junior high schools. Special education schools were designed for severely or profoundly retarded students, and those who were blind, deaf or multiply handicapped. Entrance to these schools depended on a medical model—students were tested, diagnosed and certified as eligible for special education services by medical doctors, often at an exam given to 6-year-olds prior to entering compulsory education, rather than by an educator-based team as in the United States. Special education schools were not designed for students with relatively minor disabilities such as ADD, LD or autistic spectrum disorders, which went essentially unrecognized.

There were special high schools, but no special classes in regular high schools, because students attend schools that are designed to be consistent with their achievement level, based on their scores on admission examinations. High-school-aged students with disabilities often went to vocational schools or night schools after work, rather than to academic high schools. There were no minimum competency requirements for receiving a diploma from the special education system; there was essentially no dropping out; and virtually all students completed an upper secondary school program. 
Students with significant behavior problems or those whose behavior brought them into the legal system were sent to separate schools, where teachers frequently used behavior modification or "body relaxing" techniques to improve behavior. These students were rarely returned to the public school system. Thus, there were few serious behavioral problems in general education, although parents frequently complained that discipline was deteriorating.

There were some separate classes within mainstream schools for students who were "school refusers," sometimes called "counseling" or "guidance" classes.

Approximately $0.5 \%$ of elementary and $3.3 \%$ of junior high school students were denoted "school refusers." A significant number of the school refusers, identified as those who miss more than thirty days per year (other than for illness), may well be those who have difficulty keeping up with their peers.

\section{Incidence/Identification of Disabilities}

Overall, Japan has identified a relatively small number of students for special education. There are several reasons why fewer students are identified in Japan than in many other developed countries. These reasons include a cultural distaste for separating students from the group and perceptions by teachers that learning issues are due to their own failures and, with a little bit of extra help and hard work, everyone can succeed, and a basic belief that there are fewer children with disabilities in Japan because of good medical care and the availability of legal abortions.

It appears that there is a significant difference between how American and Japanese educators perceive disabilities, and therefore how many children they identify. For example, Haynes, et al. (Haynes, 2000) observed that American elementary school teachers identified $4.0 \%$ of their students as having learning disabilities, defined by the 
study as a two-year lag behind their age peers, while their Japanese counterparts identified 1.5\%. More United States teachers identified disabilities in listening, speaking, reading, writing and study skills, while more Japanese teachers attributed school difficulties to weakness in social skills. This difference appears consistent with Japanese emphasis on well-defined rules for social interaction and cooperation among peers.

A similar finding was reported by E. Marcia Sheridan (Sheridan, 1993), who compared the rates of identification of reading disabilities in Japan and the United States. She found that Japanese educators generally cited a very low percent of students as having reading disabilities, although international tests appeared to indicate that reading problems affect about the same percent of students in countries with very different alphabet structures. She suggested that Japanese teachers and parents were more likely than Americans to attribute a reading problem to lack of effort or improper teaching than to a "reading disability," and may see a reading problem as something temporary that could be overcome through additional work, rather than as a lasting disability.

Another way of looking at the low identification rate is the possibility that, indeed, there are fewer students with disabilities in Japan than in other similarly developed countries. Shigeru Narita (Narita, 1992) attributed the relatively small number (less than $1 \%$ ) of Japanese children receiving special education services, in part, to the low infant mortality rate and excellent prenatal care available to Japanese parents. The availability of legal abortion, and its use to abort babies who might have been born with significant disabilities, also affects the number of children needing special education services. It does appear, from data reported in other countries, that the number of children identified with 
low incidence disabilities decreases with high quality medical care, but that numbers of students with the less visible, high incidence disabilities may not be significantly affected.

\section{Teacher Training for Special Education}

As of 1993, it appeared that training in special education for teaching

professionals was improving, but that it was still not adequate to match the significant growth in the preceding 20 years in the number of students identified for special education and taught primarily in special schools and classes. Giichi Masawa and colleagues (Masawa, Seo, \& Kusanagi, 1993) found that a certification for teaching special education required courses in addition to those required for general education, typically in the specific disability to be taught, such as blindness or deafness. There were a very small number of master's degree programs geared to teaching in the special schools and a new nationally-funded internship program for newly certified teachers. There were no speech therapists, or physical or occupational therapists, in either regular or special education schools. Thus teachers of children with disabilities often had to serve the role of therapist as well as teacher. Teachers of special education classes were paid a stipend of 6-8\% above their regular teacher salaries, although only one-third of teachers in special classes were certified in special education. Education for the "learning disabled" had not been incorporated into the curriculum, and there was no specific training for teachers in the area of learning disabilities.

\section{Special Education in Early Childhood}

In the period between the 1990's and 2005, students with disabilities tended to be fully integrated into early childhood programs, even though most special education in the compulsory schools was separate. During the 1980's and 1990's, the population of children had decreased markedly in Japan, which led kindergartens and day care centers 
to admit more children with disabilities to fill their spaces. Makato Tsumori (Tsumori, 1998), who directed preschool education programs for children with disabilities as early as 1949 , believed that this resulted in greater understanding that children with disabilities were not different from their peers in terms of their humanity and feelings, and that young children with disabilities should not be separated from their families and placed in special schools.

There were, Tsumori observed, few or no early intervention or organized identification programs for special education before school age, even for those who were significantly handicapped or cognitively disabled and, in general, all children played together and went to preschools together. It was the parents' choice to place their preschool children in a special school, special classroom or regular education classroom. Parents often chose education in a regular classroom with little support, rather than separation from the child's peer group.

\section{Visual Impairment}

Most visually impaired students in Japan were educated in special schools or in “visual impairment units” within regular education schools. As of 1995, there were 70 special schools for visually impaired students and 98 regular schools with visual impairment units. Students in special schools were required to be educated in Braille. Kakizawa (Kakizawa, Douglas, \& Kagawa, 2000) also found that the number of visually impaired students served had decreased from its peak in the late 1950's because of a decrease in total school age population and a decrease in prevalence of visual impairment from 27:100,000 to $16: 100,000$ from 1970 to 1991 .

While approximately $36 \%$ of sighted students entered university after completing upper secondary school, only 20-30 out of the approximately 7000 students served in 
schools and special classes for the visually impaired (about $0.4 \%$ ) entered university studies. M.E. Wong (Wong, 2004) attributed this discrepancy, even in a society that strongly values educational credentials, to the tradition of riryoka (acupuncture and massage). Since the Edo period (1603-1867), riryoka positions were allocated to blind persons and, in more recent times, training for these jobs occurred within schools for the blind. In addition, there was a government prohibition against other institutions training people in these skills, to protect the jobs of individuals with visual impairments.

\section{Special Education Since 2005}

It appears that, despite major changes in law and some real changes in the discourse surrounding special education since 2005, changes at the school and class level have been very slow. The great majority of students with significant disabilities are still served in separate settings, with a few small experiments in "integration," and the great majority of those with high incidence disabilities are still served in regular classrooms, with relatively minimal increases in available supports.

An initial report, "Special Support Education in the Future," was published in 2003, defining "special support education" to include those children with learning disabilities (LD), attention deficit disorder (ADD) and high functioning autism. It stated that special education would be offered according to a student's individual needs, and that special education would not be defined by attendance in a specific class or school. In 2007, the new system was adopted as an amendment to the School Education Law as Special Support Education. Students with LD, ADD and high functioning autism were now officially entitled to receive special education services under a category of "developmental disabilities." 
But a new law does not always mean real change in practice, especially when what is changed is primarily definitions and not specific requirements for how students are to be educated. One author, Yoshihiko Goto (Goto, 2008), wrote a critique of the 2007 Special Education Law in Disability Studies Quarterly, in which he stated that he expected serious problems with this new policy. Although the new law was designed to increase normalization and was to be a catalyst toward equal opportunity, based on the history and social context of special education in Japan, Goto was not hopeful that there would be much substantive change as a result of this new law.

Misa Kayama (Kayama \& Haight, 2012) also observed that the 2007 law recognizing the need for and establishing special education services for students with high incidence disabilities created a problem for educators in Japan, who now had to blend the new law with traditional approaches to education and socialization.

Under the new law, schools would be required to provide specialized support for students with LD, ADD or high functioning autism. Each child would be assigned a special education coordinator, who would coordinate with parents and teachers to provide individualized assistance to students who are struggling in general education classrooms. This support may range from extra help within the classroom to assistance in a special classroom for all or part of the day. Parents and educators would have to balance these new practices, similar to those required by United States special education law, with traditional practices and beliefs about educating children.

Kayama observed that a significant challenge to implementing the new, more formal special education procedures was the reluctance of adults, particularly parents, to do anything that might stigmatize children. In Japanese society, anyone who is a little bit 
different is perceived as a "stranger." Therefore, it would be difficult for parents to accept that their children had disabilities and even more difficult for parents to give permission for them to receive special services. Even those who had accepted that their children had disabilities would likely be very concerned about other people's potential negative reactions and might feel shame that their children were not "normal." Some parents would respond to the stigma of having a child who was different by denying or hiding their child's disability.

In Kayama's study, teachers also recognized the need for gradual transitions in parents' understanding and willingness to accept a child's placement in a resource or special education room. Teachers worked with parents to increase their readiness to accept their children's separation from the group, and to create an environment of trust, empathy and belonging for both parents and students. Similarly, the teachers focused on slow, careful transitions in students' understanding, considering their developmental levels and readiness. Some children receiving support recognized that they were somehow different from most of their peers, but not that they "had disabilities," and parents were generally not willing to discuss a "diagnosis" with their children.

\section{Incidence/Identification of Disabilities}

Even under the new law, which specifically includes the high incidence disabilities of LD, ADD and high functioning autism, Japan continues to identify far fewer students than the United States or other developed countries. Yui Murakami and Heinz-Dieter Meyer (Murakami \& Meyer, 2010b) looked at the reasons underlying the markedly lower incidence of reported disability in Japan, which they attributed to “Japan's strong collectivist and paternalistic orientation and the requisite institutions promulgating those beliefs" (p. 202). As of 2005, the United States identified 
approximately $14 \%$ of its students as having “special needs," whereas Japan identified 1.6\%. As compared with the United States, which adopted a "human rights approach," the authors consider Japan's approach to disability to be "paternalistic." It continues to be based on a medical model, where individuals and/or their parents defer to authorities, who determine where they go to school and what they learn based on a medical "diagnosis." Parents tend to accept the word of medical or educational "experts" and there is virtually never any legal action relating to a student's placement.

Further, the decision that a child is eligible for special education typically segregates him/her from the "normal" population in what the authors describe as a "once and for all" placement decision based on medical, rather than educational, criteria at the start of compulsory school. There is a strong incentive to keep the number of such children low, so children with "minor" or "invisible" disabilities are channeled into the "normal" mainstream classes. After the initial screening, at first grade, there are few changes in student status, both those in special education or those in regular classes. So problems, such as learning disabilities, as well as problems with socialization that tend to manifest only after academic education has begun, are typically not recognized, and students with those issues remain in regular education with their special needs unaddressed.

Murakami and Meyer noted that there is far less individualization in Japan than in the United States, both within special education classes and schools and in the mainstream. There appears to be an assumption that a student who is placed properly, either in a typical classroom or, based on his/her disability, in a special setting, is getting what he or she needs and does not need additional individualization, although very 
recently the idea of the Individualized Education Program (IEP) has been introduced to Japan through the 2007 law, but has not yet been implemented to any degree.

Nevertheless, the authors noted that, in the last 20 years, Japanese policies toward disability have made major strides toward conformity with Western nations' policies. A major change has been a de-emphasis on institutionalization and a move toward individuals with disabilities living in the community, at least partially resulting from the rise of grass-roots organizations pushing for greater inclusion and increased services.

\section{Mental Health Issues}

Amy Borovoy (Borovoy, 2008), who studied the kikikomori children, those not attending any school, was particularly concerned with how difference is managed in Japanese society, which historically prioritizes inclusion and mainstreaming over human rights, and how that affects the existence of so many children outside the educational system.

Japan has had what Borovoy called a "minimalist" outpatient mental health system, with very few independent therapists or clinics, and anti-depressive drugs are hardly used. Borovoy reported that Japan has an extremely low rate of self-reported mental illness, and few Japanese seek help from mental health professionals. Mental health problems are considered, at least partially, to be voluntary conditions, and treatment often consists of improving social skills and accepting social values, rather than diagnosing or treating mental pathology by drugs or psychological counseling. Where counseling is offered, it often focuses on behavior modification-altering life patterns, returning to work or school, regulating sleep, or returning to one's role as a family member. 
Japanese attitudes toward mental illness appear to be rooted in the Japanese concept of equality, and the idea that success is the result of effort rather than ability. The purpose of education is seen as socialization into shared values. Although Japanese teachers visit students' homes and advise on daily life habits and study routines, they consider a discussion of family relationships and individual psychological problems to be "off limits." The pressure to hide problems and fit into the mainstream leads parents to insist that their children be placed in regular classrooms, even when their needs might be served better elsewhere.

\section{Parent-School Interactions}

There is a much greater stigma in Japan than in the United States when a child is identified as having a disability. Disabilities still tend to be regarded as abnormal, and many people still regard the presence of a disabled person as a source of shame for their family. It is still widely believed that care for individuals with disabilities is primarily a family responsibility. The belief, generally held in the United States, that it is the obligation of government and schools to support and accommodate individual rights of people with disabilities, and to provide opportunities for equal participation, is not prevalent in Japan, where parents feel that asking for help is asking for a "favor." These beliefs have resulted in an approach to disabilities in Japan that labels very few children as disabled, mostly those with low incidence, severe disabilities, and educates them separately.

In both Japan and the United States, parents regard the relationships they establish with professionals who provide services to their children as very important. Misa Kayama (Kayama, 2010) studied parent-school relationships in both countries. When the Japanese special education system served only students with severe disabilities, there was 
very little parent involvement in decision-making, so regular communication between professionals providing special education services is new to Japan. Japanese parents now report more frequent negative experiences with professionals, perhaps because they are hesitant to communicate due to what Kayama describes as the "hierarchical relationships" in schools, as well as because of the perceived stigma. Parents see themselves as possessing less power than teachers, and are uncomfortable when challenging or disagreeing with them. They also express the fear that, once they disagree with a teacher, the teacher might not give proper care to their children. Japanese parents, in particular, value empathy in their interactions with professionals, and look for such expressions from professionals before they feel comfortable speaking up. Japanese parents voice concern that that there is little help available with transitions from one school level to another, unlike in the United States, where procedures regarding transitions from one level or school to another and from school to community are clearly codified.

\section{Mobility Problems and Lack of Facilities}

In the United States, under IDEA, special education services are limited, by definition, to those disabilities that interfere with learning. Other disability issues, such as accommodations for mobility problems, including ramps, are covered under Section 504 of the Rehabilitation Act. But in Japan, individuals with mobility problems are assigned to special education schools even if there are no learning issues. In fact, because Japanese schools are generally not accessible and are often multi-story, students with mobility problems often cannot be accommodated.

\section{Legal and Policy Factors}

Under the Japanese Constitution's Fundamental Law of Education, special education is covered within the general framework of the education system, and thus is 
considered a fundamental right. The current Ministry of Education, Culture, Sports, Science and Technology (MEXT) web page on Special Needs Education (Ministry of Education, Culture, Sports, Science and Technology (MEXT), 2008) defines special needs education as education for students with disabilities. Students in need of special education are defined by a categorical system that depends on identification of a child's disability, rather than on the personalized educational needs of individual children. There are currently seven categories of disability: blind or partially sighted, deaf or hard of hearing, mentally retarded, physically disabled, health impaired or physically weak, speech impaired, and emotionally disturbed. The special education law addresses implementation of special education and the duty of each prefecture to establish special schools. As it does for regular schools, MEXT prepares courses of study that include the instructional content for special schools. MEXT also sets the size of classes in special schools and for separate classes within regular schools, at six students per class in special schools (three for multiply-handicapped children). Special schools at the upper secondary level may have eight students per class (three for multiply-handicapped children).

In 2012, Mayumi Nagano and Lois Weinberg (Nagano \& Weinberg, 2012) collaborated in a study and review of special education laws and court cases relating to education of students with disabilities in Japan and the United States. In the United States, under IDEA, there is a clear statutory preference for inclusion, specifically placing students in the least restrictive environment (LRE) consistent with educational and physical needs. There is a clear requirement that students with disabilities receive any related services that may be required for them to benefit from a placement in such an environment. American case law has established criteria for determining whether 
placement in a mainstream school or class is appropriate, and federal guidelines clarify the related services that are required for a student who attends a regular public school.

The authors reported that, as of 2006, parents of children with disabilities in Japan were just beginning to file court cases to achieve placement for children with healthrelated needs (such as use of wheelchairs) in regular schools and that, more generally, there are still very few court cases related to placement or education of students with disabilities, particularly "minor disabilities," such as learning differences or attention problems. The authors explain this difference by citing the difference between Japan and the United States in the way each society handles difficult situations. In the United States people tend to resolve conflict by confronting each other, in person or through legal process, while in Japan, people tend to deal with conflict by avoiding confrontation. In the United States, there are about 3000 due process hearings under IDEA annually, of which $10 \%$ become court cases; in Japan, as of 2012, there were only 67 cases since World War II.

\section{Laws and Government Policy Related to Special Education}

The School Education Act, enacted in 1947, shortly after the end of World War II, did not include a statement defining special education; however, the 1949 Ministry of Education Establishment Act followed what was then the American model, and defined "special education" in terms of where students are served (in special education schools), rather than in terms of students' needs. By 1971, the Amendment to the Ministry of Education Act included education in special classes within regular schools. In 1979, Compulsory Special Education was implemented, mandating education for students with disabilities in grades 1-9. It created a separate system of special education in special schools or separate classes to provide that education. By 1993, the Special Education Act 
added resource rooms, allowing for the possibility that some students in regular classes could also receive services in a special room within their school. As of 2002, students with severe disabilities could be placed in regular education schools under an exception granted by local education boards. The 2007 Special Needs Education Law created a major change, by including students with so-called "high incidence disabilities," such as learning disabilities (LD), attention disorders (ADD and ADHD) and high functioning autism, and provided for their education in regular schools. However, the new system still does not assure the rights of students with disabilities, and does not contain the concept of inclusion or LRE (Least Restrictive Environment).

The 2009 School Education Act requires that education be provided in special needs schools for visual and hearing impairment, mental disorders, physical disabilities and other health problems. Professionals from those special needs schools are also to advise regular schools, if requested, on education of students with special needs. The Act also provides for special needs classrooms within regular schools for students with moderate hearing or vision disorders, mental disorders, or physical or health-related disabilities. Thus, this Act appears to be based on the premise that students with disabilities should be educated in special schools or special classes. This Act does not specifically mention or provide for high incidence disabilities. Local education boards are to notify parents of students with low incidence disabilities of whether they will be assigned to special schools or will be permitted, under special exception, to attend regular schools. Nagano and Weinberg (Nagano \& Weinberg, 2012) noted that these provisions differ from the presumptions under IDEA that children will be mainstreamed unless the 
placement is inappropriate. In Japan, the assumption is that students will be separated, unless an exception is granted.

Beginning with the enactment of the School Health and Safety Act in 1959, all children entering compulsory school receive a physical examination. If, based on that examination, a child is found to have disabilities, a committee reviews the child's school placement and assigns the student to a regular or special school. There are no special education classes in either kindergartens or high schools, so most children at those levels with low incidence disabilities are placed in special schools. In 2004, it became a requirement under the Enforcement Order for the School Education Act that the Schooling Guidance Committees hear parents' preferences, but parents still may not participate in the decision process.

Resource rooms have been set up in many compulsory-level schools to serve students in regular classrooms who may have less severe speech disorders, autism, mild hearing or vision impairments, $\mathrm{LD}$, or ADHD, but these resource rooms may be in schools outside the student's neighborhood. There is no legal requirement to provide services to such students.

Based on the data in the 2012 paper by Nagano and Weinberg (Nagano \& Weinberg, 2012), only $2.2 \%$ of the total number of students in compulsory education are currently identified as having disabilities. Of these, approximately $25 \%$ are in special schools, $54 \%$ are in special classes and $21 \%$ are receiving some education from resource rooms. The authors point out that this means that $79 \%$ of those identified with disabilities are placed in special schools and classes. Nagano and Weinberg reported that Japan estimates that $6.3 \%$ of students have disabilities, if the students with high incidence 
disabilities covered by the 2007 Special Needs Education Law are included. The number of students who enter regular schools as "exceptions" is about $0.9 \%$ of the total number of students in special education.

The authors pointed out the absence of the language of "human rights" in Japanese education documents. Unlike in the United States, there is little talk about the rights of either students or parents, and the concepts of "normalization" and "equal access" used in the United States are reframed to stress independence and community involvement of individuals with disabilities.

However, the authors saw several barriers to inclusion in Japanese special education law:

1. The LRE concept does not exist in Japanese law or practice. Under Japanese education law, it is presumed that students with disabilities are to be educated in separate settings. Only after it has been determined that the student can be educated in a regular setting with no additional support can a student be admitted to a regular school as an exception.

2. In the authors' view, rights for both parents and students with disabilities are weak; the legal basis for supporting and assisting students with disabilities within regular classrooms is inadequate. Further, the decision of whether a student is placed in a separate or regular setting is made by the school principal; the student or his/her parents have no rights in the decision-making process. There are few procedural safeguards and thus parents rarely argue for a change in placement.

3. Because Japanese law does not require support for children with disabilities in regular classrooms, parents are sometimes asked to come to class on a regular basis to support them, and children are sometimes denied placement in a regular class if the parents cannot do so because of other obligations. In practice, without the requirement for special support, students with less severe disabilities are likely to be placed in regular classrooms, but without additional support. Currently, regular teachers or other students in a class provide services if family members of the student with disability cannot come to school to provide the necessary care or support. 
Nagano and Weinberg also pointed out that there is a significant problem of underidentification — the number of students who could benefit from special services within the regular classroom is probably substantially higher than the number of students currently identified, based on the fact that the total percent of students identified as needing some kind of assistance is far lower in Japan than in most other developed countries, including the United States.

A 2001 MEXT committee issued five proposals to improve education for students with severe disabilities and provide support for children with higher incidence disabilities:

1. Develop individual support plans for all students with disabilities, and involve parents in their construction. These plans have started to be developed, but are not required by law; there is no requirement that such plans be implemented. Further, there is no requirement for parent involvement in creating these plans and, in practice, parents have generally not been involved.

2. Assign special needs education coordinators in all compulsory schools. Over $80 \%$ of schools have assigned special needs coordinators, but they generally are not specially trained. More often, they are regular education teachers assigned an additional task.

3. Improve collaboration among those providing special education services.

4. Change special education schools into "special needs centers" that will provide training for general education teachers and will consult with parents. The authors suggest it is unfortunate that this new function for staff of special needs schools was added to the already difficult one of educating students with severe disabilities.

5. Alter both special education classes and the resource room system to provide services to children with disabilities attending regular classrooms. This affects primarily children with higher incidence disabilities since very few children with more severe difficulties attend regular schools as exceptions.

Based on their analysis, Nagano and Weinberg suggested three issues that Japan should address in order to move toward greater inclusion: creating a legal requirement to 
make LRE the default; increasing rights of students with disabilities and their parents; and increasing support services for students in regular classrooms. They noted that it is not simply changes in law, but social reform and changes in school system culture that are needed.

\section{Significant Special Education Case Law}

Three articles in Japan's Constitution are frequently cited in the relatively small number of special education-related court cases. Article 13 assures the rights of life, liberty and the pursuit of happiness; Article 14 bars discrimination in political, social or economic arenas based on race, sex, creed, social status or family origin (disability is not listed specifically); Article 26 assures students the right to an equal education. Since special education laws do not contain specific provisions about rights of students with disabilities, those pursuing court relief generally rely on one or more of these constitutional provisions. Some commentators have suggested that Article 26 supports separate education for those with disabilities, and does not provide a good basis for an argument for inclusion in regular education classes.

In Yamazaki v. Rumoi Board of Education (1993), Megumi Yamazaki, who used a wheelchair as a result of a spinal injury, was admitted to a neighborhood elementary school with the requirement that a family member serve as her aide in school. When her parents could no longer come to school regularly, she was told she could not stay in the regular school. Parents again requested admission to the neighborhood school. Despite the fact that the parents wanted her placed in a regular classroom, the school board refused and placed her in a special classroom. The parents filed a suit in the district court, alleging that the decision not to place Megumi in a regular class violated her constitutional rights. The Board of Education argued that decisions on placement should 
be made entirely by administrators, and parents had no right to choose placement for their child. They said the Japanese Constitution did not require that students receive compulsory education in regular education classes. The court ruled for the Board of Education. In a decision that the authors noted is reminiscent of the 1896 "separate but equal" Plessy v. Ferguson decision in the United States, the court ruled that it is not illegal for students with disabilities to receive the same general education in a different place. According to the authors, this case called attention to the fact that Japanese special education law is ambiguous and does not adequately protect the rights of students and their parents.

Aoki v. City of Higashiyamato (2006) considered Suzuki Aoki, a five-year-old girl who was born with a throat disease that required a tracheal tube that needed hourly suctioning. Her parents wanted her to attend a regular preschool, but the city refused, saying that the school could not care for her medical needs. The court ruled under the Child Welfare Act, that even with her disability, Suzuki Aoki was not that different from other children, and therefore could be accommodated in a regular classroom. Suzuki, they ruled, could take care of her medical needs herself, and was otherwise meeting typical developmental and cognitive benchmarks. Although the Aoki case was a Child Welfare Act case, the court's reasoning may become be a precedent for education cases. However, the court's decision does not require that regular Japanese schools provide health-related services. In the United States, school districts are required to provide health-related services in regular schools, provided that they can be carried out by trained laypeople or a school nurse. American children are not required to meet cognitive or developmental 
benchmarks or to prove they can care for their own health needs in order to be placed in a regular school.

In Taniguchi v. Town of Shimoichi (2009), Meika Taniguchi, a 12-year-old girl who used a wheelchair as a result of cerebral palsy, was initially placed in a regular elementary classroom with additional support, but was refused attendance at the local junior high school. She was found eligible for special education, and therefore for placement in a special school, and was told she did not qualify for an exception to attend a regular school. The school board of Shimoichi said that the regular junior high school could not accommodate a wheelchair and did not have personnel available to support a student who used a wheelchair. The court addressed whether Meika Taniguchi's situation would make her eligible for attendance at the regular junior high school as an exception under the Enforcement Order for School Education Act. The court ruled she was eligible because she could be educated in a regular class without any modifications to the building. The court agreed that municipal education boards have the right to decide whether students are eligible for attendance in regular schools, but they criticized the Board of Education for the refusal of this girl's request to attend the local school without making efforts to meet her needs, given the specific nature of this student's disability, the school facilities and available school personnel. This decision did not address inclusion because the family never asked that Meika Taniguchi be placed in a regular classroom, only in a special class in a regular school.

While this is likely to become a landmark case for special education in Japan, the court supported the School Education Act of 2009, with its assumption that students with disabilities are to be educated in special schools unless they qualify for an exception as 
defined by the local education authority. Given this assumption, it continues to be very difficult to qualify as an exception and be admitted to a regular school. The court did not state that the preference for placement should always or even generally be the LRE. It is difficult for students to qualify for exceptions because Japanese schools are generally not barrier-free, so that accommodation for students with physical or medical disabilities is difficult, requiring special personnel, services or construction.

\section{Summary}

This chapter examines the historical, cultural, political and legal bases for special education policy in Japan. The first part of the chapter focuses on the general education system, where the vast majority of students with high incidence disabilities are served. Since very few students are formally identified, this study assumes that there are a substantial number of students with "minor" learning problems who receive no formal assistance, but who are placed in general education classrooms with few, if any modifications or accommodations. The second part of the chapter addresses special education. To give historical and cultural context, it begins with a discussion of the development of special education policy and practice in Japan from the pre-World War II period through the post-War American Occupation, up to the period since 2005, and then summarizes special education laws and the small number of legal cases related to special education. Most of those cases are brought by parents seeking an exception for their children to attend regular schools rather than the special education schools to which they were assigned. While the courts sometimes sided with the parents, they appeared to uphold the principle that, once a student is deemed to be "disabled," and is assigned to a special setting, the student (or his/her family), must prove that the student can function in a regular school in order to receive an exception to that placement. 
In the post-War period, American educators set up a system of separate schools for students with low incidence disabilities, paralleling what was then the state of special education in the United States. It provided, for the first time, opportunities for students with significant disabilities to receive an education. But services for students with high incidence disabilities were largely absent until the last ten years or so. Within that time, Japan has adopted laws that follow very closely the definitions used in the United States, and that aim to provide services for students with higher incidence disabilities, such as $\mathrm{LD}, \mathrm{ADD} / \mathrm{ADHD}$, and high functioning autism.

But there is still substantial resistance to full implementation of the new law, based in part on clashes with cultural values that emphasize effort over ability, and stigmatize separation from the group. Students are still assigned to separate schools or classes, with little decision-making input by parents and with no presumption of inclusion or placement in a least restrictive environment. In fact, students, once diagnosed with a disability, have to demonstrate that they can function in a regular school environment in order to be awarded an "exception" from their placement in a special school. The lack of any requirement that schools be barrier-free means that students with physical disabilities often cannot be accommodated in regular schools, even if they have no cognitive issues that would make their education in regular classrooms difficult. Because there is no human rights language in Japanese education law, and because Japanese culture tends to deal with difficult situations by finding ways to avoid confrontation, there are very few cases that challenge the presumption of separate education or assert the rights of children to receive services, and of those few, the courts have tended to uphold the right of schools to assign students to separate settings, and to make inclusion an "exception." Japan 
continues to identify a much smaller percent of its students with disabilities than most developed countries, and education at special schools for the disabled appears to be highly developed.

While Japan has been very successful at educating the great majority of its students without providing a lot of special services, critics have noted that there are students who could be served in regular schools and classes, who are isolated in separate schools, and that there are students with high incidence disabilities who could benefit from additional assistance that they do not currently receive. 


\section{Chapter VI: Discussion, Analysis and Conclusions}

\section{Introduction}

This dissertation focuses on the cultural, historical and policy/political and legal elements of Swedish and Japanese special education policy and practice that has enabled each country to experience significant success in some or all of the metrics of success discussed in Chapter II. The ultimate goal of the study has been to explore what the experiences of Japan and Sweden have to tell us about special education policy and practice in the United States.

This final chapter returns to the research questions enumerated in Chapter I, and seeks to provide answers to these questions:

I. What policies, initiatives, and educational practices in Japan and Sweden lead to successful education of students in Japan and Sweden?

II. What are the cultural and historical factors that enable Sweden and Japan to experience success with the great majority of students?

III. What are the policy/political factors that enable Sweden and Japan to achieve success with the great majority of students?

IV. What legal factors and approaches enable Sweden and Japan to achieve success with the great majority of students?

V. Why is special education more "successful" in Sweden and Japan than in the United States (as measured by metrics of success such as limiting costs and legal burdens, to maximize the ratio of benefits to costs; high school graduation rates; 
academic achievement; preparation for jobs, independence and continuing education; fairness and financial incentives and disincentives.

The discussion of the findings of this dissertation relative to the research questions is organized in five sections. The first and second sections review and summarize the cultural, historical, policy and legal factors that seem to have a strong influence on the success that Sweden and Japan, respectively, have achieved in some or all of the success metrics. The third section looks at common themes and areas of difference and examines what effect these might have on the kinds of successes and challenges each country has experienced. It then recommends some areas where Sweden and Japan might look at their own programs. The fourth section examines what the United States special education community might learn from the examples of Sweden and Japan. The final section offers conclusions, revisits limitations of the study and offers opportunities for extending this research.

\section{Cultural, Historical, Policy and Legal Factors Affecting Success in Swedish Special Education}

\section{Introduction}

Sweden's successes in special education are focused principally on the metrics that are concerned with high school graduation rates, preparation for jobs, independence and continuing education and fairness rather than on achieving very high levels of academic success. Sweden's approach to special education is particularly notable for its early embrace of the principal of "normalization," not just for those with high incidence disabilities, but for those who are most severely afflicted. That principle extends not just to inclusion, wherever possible, in the comprehensive school, but to the nature of living arrangements, family life and participation in the economy as job holders. While culture 
underlies the broad social and educational policies that have led to that success, it is Sweden's broad social service policy and its seamless integration with education policy that have bolstered both normalization and Sweden's approach to dealing with students with high incidence disabilities. Sweden's policy of giving assistance to any student who needs it, for a long or short time, without the administrative requirement for identification, is at the center of Sweden's success. So is, it appears, its built-in policy allowing flexible time and alternate routes to achieving what might otherwise be a difficult-to-reach and rigorous standard for qualifying for upper secondary school, graduation certificates or entrance into tertiary education.

\section{Cultural and Historical Factors}

\section{Culture of Social Welfare}

Until the 1990's, Sweden appeared to have a very well-developed approach to children with special education needs that meshed well with its cultural heritage. Sweden had long nurtured a communal vision of society, a strong commitment to social welfare that relied on a broadly accepted high level of taxation in exchange for generous family, health and education provisions, under a highly centralized federal government. This support for families makes possible normalization for those with significant disabilities, but it also supports success for students with relatively minor disabilities.

\section{Accommodating Diversity Within a "School for All"}

The concept of a "school for all," the idea that, increasingly, most educational diversity could be served within one school, at least through compulsory schooling, is ingrained in the cultural fiber of the nation. Control of educational curriculum and policy is highly centralized to ensure that all students, no matter where they live or their economic circumstances, receive the same education. Increasingly, in the course of much 
of the twentieth century, students with low incidence disabilities were included in regular schools, and educators celebrated the growth of all kinds of diversity within a single school model, with diversity regarded as something to be valued and accepted, and not considered deviant or abnormal. This view of diversity has formed the basis of the very successful approach to special education that relies on providing assistance to anyone who needs it, for as long as they need it, without separating them from their peer group any more than necessary.

\section{Changing School Environment to Accommodate Greater Diversity in Learning Profiles}

Swedish culture is reflected in the broadly-held idea that schools are instruments for developing individuals' potential, as well as communal organizations designed for socializing children and preparing them to take their part in the democratic society. For Swedes, it follows that the school environment should be flexible enough to accommodate the needs of many students, including students with disabilities, and that every effort should be made to create an environment where as many individuals as possible can participate fully. This approach focuses on finding ways to change the school environment, both physically and in terms of curriculum and instruction, so that students with diverse learning profiles can function, instead of using resources to make children fit the environment. It appears to be one of the reasons that Sweden has succeeded in full inclusion for large numbers of students who might be given special services elsewhere.

\section{Respect for Cultural/Linguistic Differences}

The treatment of deaf students in Sweden offers an interesting and potentially enlightening view of how Swedish culture conceptualizes and operationalizes diversity and fairness, and how it has created success in dealing with a growing cultural and 
linguistic diversity. Even in a country that appears to value inclusion and normalization, deaf students are still taught primarily in separate schools for the deaf. While it may seem that deafness is handled differently from other disabilities in Sweden, the separate placement decision for deaf students seems to be based on the same educational values as other special education decisions. Deaf students are separated because they are treated more like a cultural and linguistic minority that should be honored with a bilingual education in sign language (their native tongue) and Swedish. ${ }^{17}$

\section{Policy and Legal Factors}

\section{Absorbing Political, Economic and Social Change, Apparently Without Losing Focus on Social Welfare}

By the 1990's, Sweden's educational system began to be tested by ideas from outside Scandinavia, and was strongly affected by international economic and political forces and by Sweden's inclusion in the European Community. A new set of cultural norms, focusing on decentralization, economic efficiency and school choice, was imposed from outside its borders, and was wholeheartedly embraced, despite its apparent clash with the ongoing domestic communal culture. In addition, education had to respond to the challenges of a world-wide recession and substantial immigration, changing Sweden from a particularly homogeneous society into a multicultural one. Yet, despite mostly internal criticism, and some evidence of minor resegregation of students with disabilities, Sweden has for the most part succeeded in maintaining its vision of a school for all.

\footnotetext{
${ }^{17}$ The recent, pervasive use of cochlear implants for almost all individuals born deaf in Sweden raises new questions about whether these students are, or should continue to be, part of a cultural minority, and how their education will be treated in the future.
} 
The most surprising thing about the Swedish story is how seamlessly Sweden accepted these changes with little or no political controversy. It would appear the reason that what might seem to be a cataclysmic change was accepted so readily was that, even with that change, Sweden maintained the essence of its social welfare approach. This continued to provide a level of support and assistance for families and children far beyond anything contemplated in the United States, and perhaps is a key reason for the successes of Sweden's special education system.

\section{The Legal System is not Used as a Way of Solving Educational Disputes}

The Swedish legal system does not provide a procedure for using courts to resolve educational disputes such as placement in special education or the nature or extent of special services offered. In fact, there appears to be little discussion in the literature about how such disputes are or would be handled. The absence of this layer of confrontation from the educational placement process, with all parties appearing to be interested in what is best for the child (although perhaps with different visions of what is best), seems to contribute to cutting costs and reducing or eliminating the time lag before a student can be helped. This may be due to a greater level of confidence among Swedes than among Americans in the decisions that government entities make, but it clearly avoids a layer of contention, allowing more resources to be used for improving outcomes.

\section{Issues of Human Rights/Civil Rights/Oppression, etc.}

World-wide interest in the social construction of deviance or "otherness" has been connected, in many places, with a much larger history of oppression, by race, gender and class, as well as by disability. The struggle to keep people of "inferior" race or sex from voting has been considered parallel to keeping disabled and "feebleminded" individuals from being considered equal citizens. Unlike the relatively confrontational nature of 
dealing with these topics and the succession of legal and political battles in the United States, the approach to these issues in Sweden has been much more peaceful and has led to the wide acceptance of "normalization." To the extent that political battles and the confrontational nature of multiple legal cases have been avoided, Sweden's seemingly uncontested view of the rights of disabled persons and linguistic and national minorities has contributed to its success in realizing a great degree of normalization. It is possible, of course, that the generally accepted view of diversity as a normal characteristic of a population has contributed to the absence of political battles and legal cases regarding issues of disability and the broader issues of civil rights of all kinds.

\section{High Status and Pay for Teachers}

As a matter of policy, Sweden controls the number and training of regular and special education teachers. Teaching is a highly regarded and competitive occupation with good pay and low turnover. As in the United States, the nature of training for special educators has changed over the years, from preparing teachers as diagnosticians and remediators who work in special schools to training for the cooperative assistance and role that inclusive schools demand. The absence of the teacher shortages and high turnover typical in the United States is likely an important driver of Sweden's success.

\section{Cultural, Historical, Policy and Legal Factors Affecting Success in Japanese Special Education}

\section{Introduction}

The research described in this study appears to indicate that cultural and historical factors are more significant in establishing both the nature and the success of Japan's special education programs than those directly connected with government policy, politics, or law. Historical factors explain the adoption of some "progressive" methods in early childhood and elementary school that create avenues to success for some students 
with high incidence disabilities. Historical factors also underlie Japan's reliance on the United States model for special education law, which appears to clash with indigenous culture and thus has not been fully adopted. Cultural factors include a strong emphasis on education, building community, establishing social and group skills, defining equity as equality of the inputs of education, and reliance on perseverance rather than ability as the key to educational success. Policy and legal factors include the strongly-centralized educational system and the assumption of separation rather than inclusion for students with low incidence disabilities. They also include a tendency to solve problems without confrontation, leading to infrequent use of the judicial system, despite its availability under existing law, to solve educational fairness and placement disputes.

\section{Cultural and Historical Factors}

\section{Heritage of American Occupation}

In Japan, education policy in general, and special education policy in particular, were molded by a combination of culture and history. In 1945, at the end of World War II, the defeated and occupied Japan was essentially forced to recreate its educational system.

The new system was established in the image of the American concept of democracy by a group of educational experts sent by the United States Government as part of the occupation.

The educators sent by the United States reflected, for the most part, the thenpopular Deweyan progressive point of view. Despite the authoritarian Japanese culture, many parts of the progressive vision struck a chord with Japanese cultural values. It appears that some of Japan's evident success in educating almost all students with virtually no special provisions was due, at least in part, to the elements of progressivism that had been integrated into Japan's elementary schools. It appears also that other 
elements of Japanese culture made it possible for this paradox to occur. An underlying belief that success is the result of hard work rather than ability meant that students who struggled worked harder to meet the requirements set before them. Strong support from families for education meant that families of struggling students saw it as their duty to provide the extra education needed for their children to succeed. The juku system responded to both the needs for extra help and for parents' willingness to support their children's education as a first priority, and it filled some of the gaps in providing both remediation and enrichment.

\section{High Status and Pay for Teachers}

Another important policy/cultural factor that has influenced the success of special education in Japan is the high cultural regard, respect and related good pay for teachers, which results in competition for teaching jobs, rather than shortages, and much higher retention rates than in the United States. But, at this point, while there are trained teachers working with students with low incidence disabilities, there are relatively few teachers trained to work with the newly recognized minor disabilities of ADD, LD and high functioning autism.

\section{Cultural Elements Intrinsically Conducive to Special Education}

There are elements of Japanese culture reflected in its schools that may be intrinsically conducive to the education of students with some learning issues. An emphasis on the "whole child" and on physical, social, emotional and moral aspects of learning, as well as on the arts, may provide ways for students who do not excel easily in traditional academics to feel successful. A variety of approaches to learning, particularly in the early elementary years, that include hands-on learning and learning within a social context, may allow students who have difficulty with reading and writing an opportunity 
to access higher level ideas and take part in intellectual discussions, providing basic skills in the context of cognitive challenge, and providing continuing learning until basic skills have a chance to "catch up" with the child's potential. Learning within mixed ability, heterogeneous learning groups may allow students with weaker academic skills, but stronger skills in other areas, to learn from others at the same time that they are valued for their own contributions. The high level of organization and order creates some of the structures that are advocated as accommodations for students with organizational and executive function difficulties in the United States.

\section{Progressive Elements}

In Japan, the elements of progressive education that were incorporated over the long term are the ones that struck a chord with the existing culture, and they were incorporated with what is probably the true Deweyan understanding that there are no contradictions between progressive education, serious learning, and hard work. This model has served as the basis of extraordinarily successful elementary education. Japan has been able to educate the great majority of its children effectively, with relatively little administrative attention or extra expenditures on the education of those students who, in the United States, would be likely to be identified for special education services at great expense.

It may be that some of the progressive elements of the Japanese primary school, by their very nature, contribute to the success of these schools in educating students with high incidence learning differences such as $\mathrm{ADD} / \mathrm{HD}$, executive function disorder and specific learning disabilities within the regular classroom. Among these are the decision to maintain heterogeneous groupings throughout compulsory school, to expose all children to the same curriculum, to avoid a definition of learning differences that focused 
on a difference between ability and achievement (at least until the passage of the new education law in 2007), along with a strong commitment to the "whole child," as a social and emotional, as well as academic, being and to learning within small heterogeneous groups, where a variety of types of skills are valued.

\section{Policy and Legal Factors}

\section{Centralization/National Curriculum}

As with other attempts to transplant the product of one culture into another that was totally different, some aspects of the transplant from the United States "took," and others were, at least over time, rejected or modified to fit the receiving culture. For example, shortly after the United States occupation ended, Japanese education returned to a far more centralized pattern. Today it is very centralized, with a uniform curriculum across the country, and national examinations at the end of compulsory grades and high school, which determine students' future educational paths with relatively few second chances. The centralized system and the clear, carefully-designed nationwide curriculum appear to contribute to Japan's success in educating weak as well as strong students. The curriculum is generally sequential, aimed at mastery before going to the next skill, and tends to cover a relatively smaller number of topics in depth rather than a longer list broadly, all characteristics that have been suggested as appropriate for learners with attention and executive function problems.

\section{Special Education Law of 2007}

Several factors drove Japan toward adopting a new special education law in 2007. There was international pressure to conform to world-wide standards regarding special education services and inclusion. In addition, there was a growing concern that, despite generally high and remarkably uniform performance on international tests, Japan was 
beginning to lose its number one status. There is some evidence that there was also an awareness that some students were being left behind. Japan again looked to its long-term partner, the United States, and adopted, at least officially, most of the definitions and procedures embodied in IDEA. Although only eight years have passed since the adoption of the new law, hardly long enough to evaluate its effects fully, early reports indicate that change is slow in coming and that both educational writers and practitioners are resisting changes, in part because these changes represent a clash with deeply held cultural beliefs and values.

\section{Japanese Resistance to the 2007 Law}

The 2007 Japanese law is the first official recognition of the minor or high incidence disabilities, such as ADD/HD, LD and high-functioning autism. It adopts the American definition of disability in which identification, diagnosis, and eligibility for special services all depend on a significant disparity between ability and performance in one or more of several listed learning areas. The concept of ability is by its very nature somewhat alien to Japanese culture and educational discourse. Japanese educators tend not to view ability as an immutable characteristic of an individual as many Americans do, and thus do not compare students' ability and performance. In the Japanese view, a student's ability to perform a particular task or skill at a given time is affected far more by the amount of effort and time the student has put into mastering it than to any innate propensity for learning that skill. Thus, Japanese teachers tend not to identify students as potentially fitting into any of the new categories, and Japanese parents resist any kind of testing or identification that would set their children apart from their peer group. 


\section{Japanese View of Equality of Inputs}

Japan has been particularly resistant to adopting the American vision of special education as meeting individual needs, because the Japanese view special or pull-out programs for slow learners, the learning disabled or the gifted as unfair and unequal. To them, equality of opportunity means that all students receive the same inputs in terms of curriculum and instruction. In contrast, the American view of equality, since the early years of the twentieth century, has been to differentiate curriculum to meet the needs of individual students. The Japanese concept of equality of inputs means that it is hard for teachers and administrators to understand the American concept of accommodations, in which Americans allow some students extra time, or use of computers or calculators.

\section{Juku as a Special Education Alternative}

It is possible that a portion of the explanation for the apparent success of the Japanese elementary and junior high schools lies in the "shadow school system," the juku or "crammies," which the majority of Japanese students attend after school or on Saturdays. It appears that a significant amount of individualized instruction and help for struggling students occurs in those settings. The number of children receiving remedial services in juku are not counted in any survey of the number of Japanese children receiving special education, but they may nevertheless be receiving the kind of additional help that allows them to develop the skills to succeed in the regular classrooms in Japan's elementary and middle schools, and the literacy and numeracy skills to participate successfully in a complex, advanced society. 


\section{Common Themes and Effects on Success and Challenges in Sweden and Japan}

Both Sweden and Japan have experienced considerable, although different, successes in their special education programs. Of course, they have experienced challenges as well. It is instructive to look at some common themes and differences to see if we, as Americans, can learn from these about areas we might want to look at to improve our efforts in special education.

\section{The Use of Ability as a Metric of Comparison with Performance}

The United States has built many of the definitions it uses to determine who qualifies for special educational services on the concept of ability ${ }^{18}$ and on measuring the discrepancy between a student's ability and his/her performance in school. Although Japan, since 2007, has officially adopted similar definitions, they are rarely applied in practice because Japanese culture has traditionally not accepted the concept of ability, and has focused instead on the level of effort and perseverance to explain differences in performance.

For all but the most severely cognitively disabled students, Sweden has looked at both ability and performance more as artifacts of the social environment in which individuals live and learn - that is, the deficit or dysfunction does not lie within the child, but the school is responsible for creating an environment in which his or her difficulty does not manifest itself. In Japan, although there is little reference to the social construct approach, when teachers were asked to explain the causes of student difficulty, they most often attributed difficulties to inadequate teaching or training, to the size of classes, or to

\footnotetext{
${ }^{18}$ Because "ability" is typically defined by IQ score, which is, essentially a ratio between an individual's performance on a given skill and the performance of a student at the mean of his/her age peers, it is not truly a measure of whether or how well the student is able to perform that skill, but only of whether he or she is able to perform it about as well, or better or worse than an average student.
} 
inadequate student effort, all causes that are environmental and not intrinsic to the student (Haynes, 2000; Sheridan, 1993). Also, as Lois Peak (Peak, 1991) observed in her ethnographic study of students entering and being socialized into expected preschool behavior, teachers tend to see that a student's performance reflects the structure and context of the school environment rather than something intrinsic in the child.

The result, in both countries, is that there has been far less emphasis on measures of ability and, in different ways, an approach that rests not on a formal identification, but on whether a student needs help in a particular area or skill at a given time.

Further, United States law requires that the difficulty is caused by a specific dysfunction and that it is not the result of economic disadvantage or weak educational background. ${ }^{19}$ Disentangling the effects of dysfunction and disadvantage is generally the province of the Child Study Committees that review testing and other social and educational information and decide on a student's eligibility and the category of disability under which he or she would be served. Some commentators have noted that the fact that the learning disability category specifically excludes educational disadvantage is a significant cause of the disparities and overrepresentation of poor and minority students categorized as having mental retardation and their underrepresentation in the learning disability category (Kavale, 1988).

\footnotetext{
${ }^{19}$ For example, the IDEA defines Specific Learning Disability, by far the most frequently assigned category under United States special education law, as "A disorder in one or more of the basic psychological processes involved in understanding or in using language, spoken or written, that may manifest itself in an imperfect ability to listen, think, speak, read, write, spell, or do mathematical calculations. This term includes such conditions as perceptual disabilities, brain injury, minimal brain dysfunction, dyslexia, and developmental aphasia. This term does not include children who have learning problems that are primarily the result of visual, hearing, or motor disabilities; mental retardation; or environmental, cultural or economic disadvantage."
} 
By focusing on a child's need at a given time, rather than excluding students whose difficulties could be traced to poverty rather than neurological dysfunction, Sweden, in particular, can be far more successful at ameliorating problems.

\section{Focusing on "Educational Needs" Rather than a Categorical Approach}

In the United States, when a student is identified under IDEA, he or she is also categorized by the nature of the disability, and that category typically determines and defines the nature of placement, instruction and accommodation accorded that child. In Sweden, where few students are formally identified, the assistance a child receives is generally based on his or her specific educational needs - what changes in environment or educational supports or physical modifications are needed so that the individual can meet educational requirements, enter the job force, or live as normal a life as possiblerather than the nature of his or her disability. In Japan, for low incidence disabilities, students are categorized by their disabilities, and there has not yet been a major move to integrate students with low incidence disabilities into regular classes. Since students with high incidence disabilities still tend to remain unidentified, what help they receive in or out of school is presumably based on presenting needs, rather than on a diagnosis. For example, a teacher might give extra assistance to a student with what we would call ADD or ADHD in following the clear, consistent classroom procedures characteristic of Japanese classrooms. The ongoing training and socialization to those classroom norms would create an environment in which such a student could function adequately. To the extent that a student's needs - that is, the academic, social or procedural areas in which he or she is having difficulty — are met with educational supports matched with their needs, the approaches in Sweden and Japan, though different, would seem to be ingredients of their respective successes. 


\section{Educational and Cultural Diversity}

Sweden has long regarded differences in culture, class, gender, race, etc., as well

as differences in ability, as a normal part of the natural diversity of society. If diversity is celebrated, then it is easier to accept differences in methods or ease of learning as one of many kinds of diversity within the communal school.

The discussion of diversity vs. deviancy is prominent in Swedish literature, but hardly mentioned in Japanese articles. However, in Japan, the socialization role of schools is explicit and central. In fact, inability of students to conform and fit into the social structure of the school is cited more often by teachers when asked about school difficulties than difficulties with reading, writing or arithmetic. Although the Japanese do not use the language of diversity and deviance, the role of the school as a socializing agent, though important in all three countries, is so significant and important to Japanese education that teachers regard their major role as minimizing diversity, and students who cannot reach the socialization goals set by their society are regarded as deviant.

\section{Communalism and Individualism}

In Sweden, it has long been assumed that the community, defined as the central government, has the responsibility for meeting social welfare and educational needs, and high taxation was accepted as the price to pay for this government responsibility.

The communal goal of education as preparation for participation in a democracy is also central to the American public school concept, and one of the major reasons underlying the development of the public school system in the United States. But the United States has long espoused a very strong sense of individualism, and part of the capitalist vision is to look at the private good, that is, the value of education to each individual, to prepare him or her for a role in the capitalist society. Therefore, America, 
unlike either Sweden or Japan, is very comfortable with educational approaches that separate students based on the desire to meet their individual needs and to prepare them for their long-term economic roles in society. This has led to an acceptance of "tracking" students based on expectations of ultimate career and social status, and separation by "academic level," even as special education students are entitled to the "least restrictive environment" consistent with their educational needs. This has been a two-edged sword for American special education, where students, once identified, are entitled to services meeting their perceived needs, but also where they may the lose the opportunity for full exposure to their regular school class and to its social, verbal and analytical challenges and expectations.

One of the strands of the "progressive movement" 20 in American education was the idea of constructing schools and curricula to provide a wide variety of options, each suited to the perceived needs of different students. These differences underlie special education policy in all three countries. However, unlike the United States, where students have often been separated by ability (or performance) at relatively early ages, both Sweden and Japan have focused on keeping students together, using the same curriculum, for a relatively long time, until the end of compulsory school at grade nine, but without relaxing the uniform curriculum or the strict requirements for moving to upper secondary school and university, a characteristic which appears to be instrumental in each of those countries' success.

\footnotetext{
${ }^{20}$ Although the term "progressive movement" is often thought of as a single group of ideas, there were several different strands of progressivism. The so-called "administrative progressives" advocated the use of IQ testing to place students in different tracks according to their abilities and likely ultimate occupations, and thus provide them with an education tailored to their "educational needs." It should be noted that Deweyan progressives focused much more on the community aspects of education and favored heterogeneous groupings.
} 


\section{High Academic Standards and Special Needs}

In setting special education policy, Sweden, Japan, and the United States face an ongoing conflict between pressure to demonstrate higher academic standards and helping students with special educational needs. Both Sweden and Japan have set high, national standards, and have also included $99 \%$ of all students in their schools. Sweden has succeeded by giving help and accommodations to students and by providing alternative routes, second chances, and extended time periods to achieve the national benchmarks, and the great majority of students ultimately meet them. Japan also has emphasized high standards, and serious examinations at the end of compulsory grades and upper secondary school, although all students are admitted to, and virtually all graduate from upper secondary school. ${ }^{21}$ It appears that setting high standards, especially with flexible time lines, is not inconsistent with high graduation rates and that many students with learning differences meet the standards. Achieving a high graduation rate for special education students appears from the data presented here to be consistent with maintaining academic standards.

In the United States, attempts at rigorous standards, coupled with high stakes testing, have had both positive and negative effects. To the extent that standards are developmentally appropriate and have resulted in setting the bar and expectations higher for regular and special education students alike, and to the extent that students have succeeded in meeting the standards, they have been a positive force for preparing special education identified students for the requirements of citizenship and the job market. But to the extent that they have resulted in failure, without a reasonable opportunity for

\footnotetext{
${ }^{21}$ Upper secondary programs include everything from the most rigorous academic courses to vocational programs.
} 
subsequent chances and opportunities to learn in different ways and at a slower pace, they may well discourage students from acquiring the necessary knowledge and skills and continuing their education at least through high school graduation. As has been the case in recent years in Sweden, the move to publish scores, and to compare and "grade" schools in the United States might well lead to further separation of special education students and excluding students likely to do poorly from the pool of students to be tested.

\section{The Role of Parents and the Legal System}

The differences in focus on individual and communal needs are reflected in the role of parents and of the legal systems in Sweden, Japan, and the United States. In Japan, parents are only rarely a force for additional services for students with disabilities. Parents are often described as extremely resistant to having their children tested or identified, and averse to having their children given any special treatment that separates or differentiates them from their peer groups. There appears to be little or no individual or organized community lobbying in Japan for special services and only a few legal cases related to special education. In both Sweden and the United States, parents have been a strong, organized force for obtaining services for children with disabilities, both high and low incidence, and for changing policy as well. Even in Sweden, where there is an expectation of identical curriculum, goals and benchmarks for all students, variations in the amount of help, and sometimes the time needed to reach those goals, are fully accepted. In Sweden, as in the United States, parental involvement and sometimes pressure is understood to be a way to gain appropriate services for individual children. But unlike the United States, the Swedish legal system is virtually never used (and there is no legal process for doing so) as a way to force particular placements or to gain services for individual children. 


\section{Fairness-Inputs and Outputs of Education}

In general, a belief that fairness depends on equal educational inputs leads to exposing all children to the same subject matter in the same way, with the understanding that some children will be more successful than others in absorbing the curriculum. In contrast, a belief that fairness should focus on the outputs of education may emphasize differentiating education so that students will leave school having met a minimal acceptable standard, with the aim that all students will reach their maximum level of performance and/or capability to function in the society. Both Sweden and Japan have generally defined fairness in terms of providing a single path, or set of inputs, which included a single, centrally mandated curriculum, combined with a single measure of outputs in the form of a centralized examination system for all students. In Sweden, even private schools must follow the mandated curriculum. In Japan, because fairness is defined so clearly in terms of inputs, it has been difficult to justify providing different materials or conditions for different students, depending on their needs.

In the United States, on the other hand, the idea that inputs should be varied to maximize individual students' outcomes has been an important tenet of America education at least since the early twentieth century. In the United States, until relatively recently, education has generally been considered a very local matter, with states and often individual school districts setting curriculum, resulting in very significant differences from state to state, district to district, and even among schools within a single district. That traditional view, of course, has become a controversial political issue over at least the last 15 years. In the United States, private schools can generally set their own curricula, and their students are generally not subject to any examinations imposed by localities or states. 
The Japanese concept of equality of inputs makes it difficult to apply the principles of accommodations, which are an integral part of American special education policy. Sweden, on the other hand, has no difficulty combining the concept of accommodations with the commitment to equal educational inputs.

\section{Ameliorating Deficits and Full Access to a Common Curriculum}

In the United States, most special educators are trained to focus on remediation and skill development through such techniques as direct instruction in phonics and mathematical calculations. As a result, students in remedial groups often do not discuss the same reading books as their age peers, or cover the same curriculum in mathematics. In Japan, all students in any grade in the public schools use the same materials and discuss the same books, and little or no remediation is provided within the school for students who are having difficulty. Sweden supplements exposure to the full statemandated curriculum with a variety of different types of assistance. In the United States, and perhaps in Sweden as well, educators generally have to choose whether to use school time to teach basic skills to students who learn skills slowly, or to expose them to higher level thinking. Swedish researcher Margareta Kjellin ${ }^{22}$ (Kjellin \& Wennerström, 2006; Kjellin \& Granlund, 2006; Kjellin, 2007) explicitly raised this question when she wrote about Swedish "goals to attain" (basic skills) and "goals to aim for" (higher level thinking and analysis). It would appear that full exposure to the enriched curriculum offered to all students, combined with remediation as necessary, would be a likely prescription for success for special education students.

In every educational system, students are eventually separated into different courses or tracks to prepare them for adult occupations and educational choices. The

\footnotetext{
${ }^{22}$ This work is discussed in greater detail in the Chapter IV, on Sweden.
} 
question that differentiates one country's approach from another is when and how early in a student's career this separation occurs. Both Sweden and Japan are committed to making this separation relatively late, at the end of nine years of compulsory schooling, and in both countries, that arrangement appears to have served both regular and special education students well. Americans might find that including low and high achievers throughout the elementary and middle school years could be a way of enhancing academic success, while creating the confidence that might lead to school retention when they reach high school.

\section{Progressivism and Special Education}

Elements of the "progressive" concept played a part in the development of special education policy in both Sweden and Japan. However, because the progressive movement has many facets, in both politics and education, this term has had very different meanings in the two countries, and thus the outcomes in Sweden and Japan have been different from each other. In Japan, elements of educational progressive practice, it appears, were compatible with the native culture, and may have offered ways for students with disabilities to succeed in Japanese elementary and middle schools, particularly students with high incidence disabilities such as ADD and executive function disorder. In Sweden, on the other hand, progressivism has been more political and social than educational, leading to vast social service supports for disabled students and their families, but has similarly allowed Swedish students to be successful without formal identification.

\section{Human Rights, Social Justice and Special Education Policies}

The language of human rights and social justice appears prominently, although differently, in special education discourse in both the United States and Sweden, but rarely in Japan. In Sweden, the concept of human rights led to a general belief that 
education of students with disabilities fits entirely within the mission of the comprehensive school. An essential principle of the Swedish model of a comprehensive school is that it should be able to teach all pupils using a variety of methods and pedagogical and psychological techniques. Sweden's classical social democratic model assumes full employment and social solidarity. Education is seen as a major vehicle of social progress, justice and welfare. Compulsory schools are schools for all, regardless of class, gender or geography. Equal opportunity is defined by a common curriculum and access to a social community; free education, even at the tertiary level, is assumed without much debate.

In contrast to the vast amount of discussion of human rights in American and Swedish writing, the language of human rights is absent from Japanese education documents. Unlike in the United States, there is little talk about the rights of either students or parents, and the concepts of normalization and equal access used in the United States are absent from Japanese discourse.

\section{Normalization in Sweden, Japan and the United States}

The concept of normalization is embedded in the Swedish concept of special education. However, there is no similar term used in either Japan or the United States, although the American requirement of placing students in the least restrictive environment (LRE) is perhaps an attempt at a similar concept. Although there have been separate moves in the United States toward deinstitutionalization for some disabled individuals, unlike normalization, LRE does not extend beyond the schoolhouse to all aspects of living, and does not affect a student's life outside of school or after he or she reaches the maximum school age. 
A central feature of Japanese culture is the belief that all human beings are to be valued equally, regardless of ability. At the same time, Japanese society has had a long history of discrimination and bias against those they regard as disabled, and therefore different and "useless." In contrast to the Swedish theme of normalization and the American principle of least restrictive environment, Japanese law still assumes that education of a student with significant disabilities will be separate-in separate classes or a separate school, and students, once identified, are still required to obtain an exception in order to attend a standard public school. On the other hand, all but the 1-2\% of students with major or low incidence disabilities are fully normalized and rarely separated from students with no apparent disabilities.

\section{Differences in Levels of Identification}

American elementary school teachers identify many more of their students as having learning disabilities than do their Japanese counterparts, but Japanese teachers tend to attribute observed school difficulties to problems with social skills or accommodating to the group, while teachers from the United States more often mention difficulties with academic skills. Japanese teachers often blame themselves, rather than put the blame on difficulties inherent in the student. They mention large class sizes and multiple demands on their time, but express the belief that, if they had enough time, they could eliminate learning problems and could educate all their students. The Japanese approach to low incidence disability has been described as paternalistic, because Japanese authorities diagnose students based on a medical model, and then determine where those students go to school. Parents have little say in the placement, and tend to accept the word of the experts who make the diagnosis. 
As Lois Peak (Peak, 1991) described in her book on the experiences of how students entering preschool are acculturated into Japanese school life, teachers are hesitant to label children as "problem children" or to point out differences between what a particular child can or cannot do as compared with other children the same age. This would include a teacher's suspicion that the student is experiencing learning difficulties. Even though there is much communication between parents and teachers, problems are not often discussed with parents. Peak suggested that this is probably because it is much more important to establish a "positive and harmonious" relationship between teachers and parents than to disclose information. This is part of the cultural assumption that any issue that can potentially lead to conflict should be avoided in polite social interactions.

\section{Implications for Japan and Sweden}

Although the focus of this study is implications for the United States, a few conclusions can be drawn for special education in Japan and Sweden as well.

Japan

\section{A Potential Middle Ground for Japan that is Both Culturally Consonant and Serves Students who are Currently Struggling}

This study suggests that Japan has had difficulty assimilating the 2007 education law, which formally recognized minor disabilities and applied definitions and ideas from American special education law, at least partially because some of the ideas and requirements clashed with long-held cultural and educational beliefs. Given that Japan has a long history of success in educating the great majority of students, without the large discrepancies in achievement typically seen in comparable studies of United States performance, it would seem to make sense for Japanese educators to determine how many and which students are not currently succeeding and direct attention to them. A 
better approach might aim for a model of assistance that is culturally consonant - that separates students in need of help as little as possible from their peers, that is sensitive to the importance of social cohesion and that does not rely on ability testing and a discrepancy between ability and performance. Such a model may well provide the combination of extra help, minimal identification or labeling, and continued access to the enriched classroom environment and the extremely important social context.

\section{Inclusion and Exceptions}

Based on the findings of this study and on the experiences in the United States and Sweden, there is good reason to believe that a fair number of students now assigned to separate schools or classrooms in Japan, and barred from entry into regular schools (except in the rare cases where they apply for and are granted an exception), could reasonably rejoin the community of their age peers and succeed in a regular school. This is particularly likely for those with physical or mild cognitive deficits, who could succeed with reasonable physical or educational accommodations. It thus makes sense to question the presumption of separateness and to ask instead, more generally, what accommodations would be necessary so that a student can succeed. The absence of a requirement of physical barrier-free access in Japanese schools contributes to the difficulties of mainstreaming students with physical difficulties and it might make sense to rethink whether such a requirement would make a more normal existence possible for enough children to justify the added cost.

\section{Sweden}

\section{Compromise Between Social Aims and External Pressures}

Perhaps the biggest current issue for Sweden is finding a middle ground between the pre-1990's vision of a school for all and the post 1990's vision of a less-centralized, 
more competitive system, allowing greater choice and more local control. The greatest risk appears to be that competition, decentralization and funding formulas will turn back the gains of the previous 20 years, and will result in greater segregation of lower class, immigrant, and disabled students. It seems, however, that it makes sense to continue to monitor issues of overrepresentation of minorities, increased identification of students for special education, and increased separation of those who are identified, and to modify the reward system that allots money to school districts based on number of identified students.

\section{Finding New Ways to be Flexible}

In many ways, the Swedish system has created flexibility for students who struggle in school. These include a range of extra help and extra time to complete requirements, both while in school, and through an extensive array of post-school age options. But the Swedes have been far less flexible about modifying requirements for entering upper secondary and tertiary levels and entering specific high school and college tracks. The lack of flexibility at these levels may, in fact, limit the options for some students who might otherwise be qualified for particular careers and academic paths, for example, a student who meets the qualifications for a technical profession, but because of a disability is unable to pass the language requirement in Swedish or English. 


\section{What We Can Learn: Implications for United States Special Education}

Based on the special education successes experienced by both Sweden and Japan, although the areas of greatest strengths and continuing challenges are different, it appears that American educators can learn from an analysis of these successes and should consider incorporating the lessons through a re-evaluation of some practices that have become both culturally and legally ingrained in the American system. However, there are some obvious limitations on transferability to the United States. For example, the minimal use of the legal system in Sweden and Japan is not likely to be accepted in the more confrontational and litigious American landscape, but it might still be helpful to Americans to recognize that there are alternatives to the rather large costs, as well as the potential benefits of the way that Americans handle special education disputes.

This section outlines some of the implications of this study for United States special education, and suggests some avenues that American educators might explore as an approach to greater success on some or all of the metrics outlined in Chapter II of this study.

\section{Access to the Full, Enriched Curriculum for All Students, Balanced with Appropriate Remediation and Basic Skills Instruction}

A significant finding from this study is the high level of success, of both Swedish and Japanese schools, in each case with a much lower level of formal identification and with many fewer students (among those with high incidence disabilities) in special classes or pull-out programs than in the United States. While the particulars in the two other countries are very different, all students in each of them, at least through the compulsory period, are exposed to the same rich curriculum. The findings of this study suggest a recommendation that all students who might benefit should be exposed to the full curriculum available to those not identified as special education students. The 
observations of this study suggest that they may not only be better prepared for the higher level thinking skills needed for today's job market, but they may also be more likely to continue with and graduate from secondary school.

At the same time, those students struggling with basic skills need extra time and focused instruction to master some essentials (reading or mathematical computation, for example). Americans often group and teach students the same skills repeatedly, year after year (because they have difficulty mastering them), without giving students full access and the opportunity to show what they can do with far more demanding material. Only rarely, it appears, is there discussion of ways that students with reading problems can benefit from, for example, reading the same books, doing the same projects and participating in the same discussions as students who can read more adeptly and deeply. Japanese students, except for the few with low incidence disabilities who are taught in separate schools or classes, are all exposed (up to high school) to the same challenging curriculum and to the same higher level thinking and problem solving skills. Perhaps the Japanese have inadvertently developed an idea that works, at least through middle school level, teaching all students together so they are all exposed to the same increasingly high level material, language and breadth of vocabulary, and then using the juku, after school hours, to differentiate and give those students who need it extra help to meet the basic skills standards. In the United States, at least in most places, educators have to choose between using school time to teach basic skills to students who learn skills slowly or exposing them to higher level thinking. Some schools in the United States attempt to create the combined educational approach observed in Japan with afterschool tutoring programs, but they probably still spend more school time for struggling 
students on basic skills than on exposing all students to higher level, creative education. It is likely that success, for both Japan and Sweden, depends on creating a balance of providing remediation and reinforcement with opportunities to engage in high level thinking tasks.

\section{Perceived Needs vs. Formal Diagnosis}

In Sweden, where relatively few students with minor disabilities are formally identified, students receive help when they need it, at various times or throughout during their educational career. To the extent that help is offered in Japan, by individual teachers, by parents, or through private tutoring schools paid for by parents, it is offered, more or less, on an as-needed basis, rather than through a formal arrangement. In both countries, help seems to be based more on the presenting need(s) - for example, the child is having trouble learning to do division, or difficulty organizing himself to write a research paper - than on any global diagnosis. The United States has set up a very formal system that both ensures students with disabilities are served, but also sets the identification bar high enough (significant discrepancy between ability and performance, and neurological dysfunction) that students who may need help from time to time during their educational careers may fall through the cracks.

In addition, because assistance is frequently based on the particular diagnosis, rather than on the special educational needs of the individual, which may vary greatly among students with the same diagnosis, some students may be receiving help that does not truly match their needs. The data from this study suggest that Americans might consider adopting this "as needed" approach to helping students when and where they need it, and whether or not they have been formally identified. It would be interesting to 
see if this approach would result, ultimately, in fewer formal identifications, lower costs, and more effective outcomes.

This study also suggests that the American definitions of disability that focus on dysfunction and exclude problems related to economic, linguistic or educational disadvantage run counter to the suggestion that assistance should be based on presenting need, rather than on its underlying cause. A student who is struggling in school, not because of a demonstrable dysfunction but because of inadequate prior education, needs help, at least in the short term, as much as the student who currently qualifies under IDEA. In Sweden, such a student would receive the help he or she needs, without the necessity of identification.

\section{Educational Environment}

In both Japanese and Swedish schools, aspects of the school environment make it possible for students who might be considered disabled in another context to succeed. For example, the high level of organization in Japanese schools may well ameliorate what appears in American schools as executive dysfunction. Progressive elements, such as built-in time for physical activity and frequent group work, might limit the problems students with ADD exhibit when they are required to sit still for long periods of time. In Sweden, the question of creating an environment conducive to success for a wide range of students is explicitly discussed, and modifying schools and school procedures to include and serve the greatest number of students is a clear goal among educational policymakers.

Educational policymakers in the United States might want to decide in principle and in practice whether and to what degree it is the role of the school to adapt facilities, procedures or requirements, in order to accommodate a student who is not succeeding in 
the regular classroom. If educators believe that learning problems often result from a mismatch between the student and the environment, then it becomes the obligation of educators to create schools that minimize the number of students who are unable to function fully in that school. Through the Americans with Disabilities Act, the United States has assured that schools are accessible to students with physical disabilities, but this does not necessarily create an educational climate in which the largest possible numbers of students with educational disabilities can succeed.

In teacher training classes in special education, at least in the United States, it is often said that "special education is just good education"; that is, that all students would benefit from the environmental and educational changes that would make it possible for many more students to succeed. The Japanese experience suggests that American schools might want to re-evaluate some aspects of the practices of progressive schools and consider reincorporating those that are conducive to improving the success of students with ADD and executive function disorder. The Japanese experience also suggests that some elements of the clear and orderly classroom and the social and academic procedures in Japanese classrooms are very effective at creating an environment to help students who struggle with organization skills.

\section{High Stakes Testing}

Both Japan and Sweden have national curricula and high stakes tests for admission to high school (or specific high school programs). In Sweden, there are also very clear national standards for receiving a "pass" grade in classes. In the United States, there is no national examination for entering high school or even tertiary education (the private SAT/ACT have served in that role for some colleges, although there is now a movement away from this practice), but there is much more frequent, often high-stakes 
testing in the pre-high school grades. The existence of these tests, in a climate in which schools, teachers and districts are graded and compared on the basis of their test scores, pressures teachers and schools to identify and often exclude students from such testing. There is good evidence that, in the United States, these high-stakes standards and examinations have a significant effect on the way teachers teach, how principals lead, and how schools deal with students with special needs. It is clear that these are ongoing issues that American educators must continue to address. Sweden has had some of this experience with the introduction of school choice and the publishing of school test scores, and some commentators have suggested that some of the increased (but still low) identification of students in Sweden is caused by this phenomenon, while Japan tests all of the students in its regular schools. These experiences suggest that Americans should attempt to separate high-stakes testing from special education identification as much as possible, to remove the strong incentives for over- or mis-identification.

\section{Flexibility in Time; Second and Third Chances; Adult Education}

Sweden's practice of offering flexibility in how much time students are allowed, to achieve the national standards required to enter national programs in high school or the university, appears to be one that the United States should consider. Sweden's time flexibility is coupled with programs in upper secondary school geared to remediation in the basic subjects required for entrance into national programs, and with options for continued chances to meet the requirements, even after leaving school, through a robust, free and highly attended adult education program. Although Sweden is not flexible about the content of the requirements, its time and place flexibility offers alternatives to students who need more time to process material, or who would choose to slow down and concentrate on fewer subjects at a time. When a student has met the requirements, he or 
she has the same eligibility for high school programs or university attendance as other students who met the same requirement in a more traditional time frame. Modifying time rather than skill or competency level is a way Americans might explore to increase the achievement, graduation rates, and job readiness of some special education students.

\section{Decreasing Administrative/Legal Burden of Special Education}

This study has demonstrated that special education students could be well served without the huge administrative and legal burden, with its attendant personal, human resource and financial costs that are currently incurred under IDEA. Both Sweden and Japan, by formally identifying fewer students, other than those with low incidence disabilities, have limited the administrative costs connected with identification, record keeping, IEP committees, etc. The fact that placement and service disagreements are resolved without recourse to due process hearings and court cases also allows more money for other educational endeavors. It is true that, in Japan, once students are assigned to a separate class or school, they must prove they are eligible for an exception to enroll in a regular school, but $99 \%$ of students do, in fact, go to regular schools. In Sweden, parents have the ultimate right to ask that their children be enrolled in a regular school, whether or not educational authorities recommend a special placement. It is clear that Americans would not be willing to accept the removal of the option to ask for a hearing or to sue if their request was denied, but the research on Japan and Sweden suggests that, at the very least, the extraordinary costs should be considered, and educators should look for alternatives to costly court battles to resolve educational placement issues. Expanded use of dispute resolution techniques such as mediation and other alternatives to litigation is also worth exploring. 


\section{Limitations Revisited: Effect on this Study's Conclusions}

The analysis and conclusions presented here are based primarily on comprehensive literature searches and a reading of the relevant literature, published mainly from 1998 to 2013 . The research was supplemented by visits to Japanese classrooms and interviews with a number of Japanese educators in 2005.

Because this study was based primarily on these sources, it focuses largely on policy and practice as described in the literature and legal documents, with relatively little data on actual practice in classrooms. It is well known that practice often differs considerably from stated policy. By choices of search methods, articles to cite or discuss, and which issues to stress, this researcher has imposed her own understandings, cultural limitations and personal biases. Although virtually everyone educated in both Sweden and Japan studies English, this study was further limited by reliance on literature available in English or English translation, and there were significantly more of such sources available for Sweden than Japan. This constraint caused by language limitations, including quality and accuracy of translations available, may have inadvertently biased the range of reports and opinions available for analysis. It may be that the translation process is self-selective and that works that are self-critical are less likely to be published in English.

Perhaps the greatest threat to the accuracy and application of these findings is the danger inherent in all comparative studies - the errors that result from a researcher examining evidence from other cultures through the world view of her own culture, values and practice. This potential bias extends to the choice of research questions, the key words used for searches, the choice of articles to read, the methods of study, and the 
modes of analysis and the conclusions that are drawn. There is always concern about misinterpreting what is written and said in articles, laws and policy statements, because of personal or cultural bias, and applying distinctions that are distinctly American in the analysis, even if those concerns or distinctions are not important in the societies studied. Another problem with attempting to generalize any of the findings presented here is the ongoing question of how different countries, stakeholders, and statements of policies, use the same or similar words, but mean different things. An example comes in the discussion of such terms such as "inclusion," "integration," and "normalization," which may be used very differently in different cultures, and even by different authors from the same culture.

\section{Summary}

This final chapter has provided a review of the findings of this dissertation, focusing on the research questions posed at the start. It also has offered a number of implications for special education practice in Sweden and Japan raised by those findings. This chapter also sets forth a number of possible suggested approaches for American educators to consider when looking at ways to improve the success of American special education. These include: looking at the balance between the need to provide remediation and the need to expose students to the full curriculum; considering basing recommendations for intervention on a student's particular needs rather than on a formal diagnosis; thinking more about creating environments where a larger percent of students can prosper educationally; and looking at testing practice and at the balance between a desire for full inclusion and the need for specialized assistance. 


\section{Implications for Further Research}

This dissertation concludes by offering a number of possible directions for future research based on questions raised by the research discussed here, and designed to extend and generalize the knowledge derived from this study of special education in Sweden and Japan to a greater understanding of the processes by which nations create successful special education policy.

\section{Extend Study to Another Strongly Individualistic Nation}

Because both Sweden and Japan both exhibit educational structures that emphasize community, and focus less on meeting individual needs than on providing an equal and highly competent education for all, the question arises of whether the pattern of successes observed in these countries can be easily applied in the strongly individualistic American culture. It therefore makes sense to extend this study to one or more other countries that have a strong individualistic focus, as another source for comparison.

The example of England comes to mind because England has a similar culture focusing on individuals, and many of the same educational challenges (high levels of diversity and economic inequality, for example) found in the United States. England registers up to $20 \%$ of the total school population at one time or another during their school careers as having "special educational needs," although only about $3 \%$ of students have formal assessments and Statements of Special Educational Need. England also has a long tradition of education separated by social class, and of an educational culture that is more competitive than communal. Only in the last 30 years has the practice of separating children entering secondary school into three tracks based on their scores on the 11+ exam been somewhat modified, and "streaming" and "setting" (i.e., tracking) remain, perhaps enhanced by the current emphasis on national examination scores. The English 
system provides a continuum of service from teacher support in a mainstream class through various kinds of specialist support to full-time attendance in a special class or school, with slightly more than $1 \%$ attending special schools.

At the same time, England has chosen to define special educational needs differently than they are defined in the United States, focusing more on the nature of the observed need and less on identifying and labeling students by disability category, and thus might offer an illustrative and informative comparison. England has also chosen to include under the umbrella of special needs, difficulties caused in large part by the effects of poverty, disadvantage or immigrant status, characteristics that are specifically excluded in the United States.

\section{Extend to Other Non-Western Countries}

Another interesting alternative is to extend the study to other non-Western countries. A recent study (Kayama \& Haight, 2012) noted the lack of studies about nonWestern children with disabilities and suggested that stepping out of the sociocultural contexts Americans take for granted and studying how diverse cultures respond to disability can improve our understanding and stimulate critical thinking about how Western societies can better serve their populations, especially given the increasing diversity and pluralism of our society. Doing so may well broaden our definitions of "success," and encourage us to focus more on the social and interpersonal dimensions than the strictly academic ones.

Kayama suggested that Americans consider Asian models of "culturally sensitive" delivery of services when reviewing the structure of American programs. Americans should specifically consider: the importance of peers and interpersonal relationships in social, moral and intellectual development; the idea that classrooms are not simply places 
to learn, but second "homes" for children; the non-coercive and sensitive way of modifying behavior and adapting to the larger group within the context of everyday activities; and sensitivity and empathy in dealing with parents.

\section{Assess Benefit of Full Access to Curriculum vs. Remediation}

The research underlying the present study raised the question of the relative importance of access for all children, even those with special educational needs, to the full curriculum versus access to specific assistance in areas of difficulty. In the United States, despite legal language emphasizing placement in the least restrictive environment, emphasis has generally been placed on providing specific remedial measures to minimize deficits and/or acquire techniques to compensate for them. Less emphasis has been on giving access to the most challenging curriculum for all children. In Japan, particularly, where there are few special classes or pull-out programs, except for the most severely disabled, all children through grade 9 are exposed to the full curriculum. That seems to be generally the case in Sweden as well, although there is some significant commentary about whether students with disabilities are routinely limited in their exposure to a more challenging curriculum, by focusing on the required basic skills ("goals to attain") rather than those involving higher level skills ("goals to aim for").

Lisa Delpit, in her book, "Other People’s Children," (Delpit, 2006) talks about the importance of honoring and valuing the home culture of students, especially those from minority cultures. At the same time, she says it is essential that children from minority cultures learn to function in the majority culture. Similarly, it makes sense to provide students with disabilities both the remediation they may need and the full access to the intellectual experiences and skills offered to students with no evidence of disability. 
It therefore makes sense to construct a research study that looks at the importance of full access to challenging aspects of curriculum and of opportunities for interaction and discussion with those students who are able to access it fully. The research might look at the relative effects of: (1) exposure to the full, enriched curriculum, including higher level discussions and assignments, with no additional help; (2) exposure to the full curriculum with extra assistance, specific remediation, support with assignments, or both, whether within or outside the school day; (3) inclusion in the regular classroom, with pull-out for remedial instruction in areas of deficit, which may limit the full exposure to high level discussion; and (4) differentiated assignments within the regular classroom, with exposure to high level discussion, but assignments at whatever level is appropriate. The second of these possibilities is closest to the Japanese model, at least for the majority of students who have access to juku or assistance at home; the third, or possibly the fourth, is closest to the Swedish model.

\section{Focus More Closely on Elements Resulting in Acceptance or Rejection of Change}

In the process of looking at measures and patterns of success in special education, this study has contrasted the way two countries adapted and accepted a major cultural and political shift that has affected special education. Sweden appears to have changed substantially since the 1990's in response to national and international forces, and has appeared to make these changes with little political controversy, integrating them almost seamlessly into its educational culture. Japan, on the other hand, has resisted changes to its approach to special education over most of the last 40 years. Although, within the past ten years, Japan has adopted legislative language similar to that used in much of the developed world, including the United States, it has continued to be very slow in putting those changes into practice. 
It therefore would be very interesting to extend this study to take a closer look at the question of what cultural, political and social characteristics lend themselves to openness to accepting changes to the culture of education. This would require a more focused case study looking in detail at the way Sweden and Japan reacted to a particular change, using more interviews, observations and detailed study of what is happening at both the policymaking level and the application level, in individual schools and classes.

\section{Final Thoughts}

This study offers a demonstration of the significance of looking at other cultures as a way of improving educational practice at home. Studying how two very different cultures, those of Sweden and Japan, have dealt with the problems of educating a diverse group of students, including those with disabilities, can enable American educators to examine our nation's current practices, ask questions, and offer possible solutions that we might not have conceived or considered without an understanding of the context of practices different from our own. With that in mind, I want to express the hope and optimism that American educators will broaden their vision by including in their considerations successful approaches used elsewhere in the world, and that they will incorporate those that are most likely to be culturally consonant to improve practice in this country. 


\section{References}

Abe, Y. (1998). Special education reform in Japan. European Journal of Special Needs Education, 13(1), 86-97.

Alexandersson, M. (2011). Equivalence and choice in combination: The Swedish dilemma. Oxford Review of Education, 37(2), 195-195-214.

Allodi, M. W. (2007). Equal opportunities in educational systems: The case of Sweden. European Journal of Education, 42(1), 133-146.

Antikainen, A. (2006). In search of the Nordic model in education. Scandinavian Journal of Educational Research, 50(3), 229-243.

Arms, E., Bickett, J., \& Graf, V. (2008). Gender bias and imbalance: Girls in US special education programmes. Gender and Education, 20(4), 349-359.

Arnesen, A. L., \& Lundahl, L. (2006). Still social and democratic? Inclusive education policies in the Nordic welfare states. Scandinavian Journal of Educational Research, $50(3), 285-300$.

Aron, L., \& Loprest, P. (2012). Disability and the education system. The Future of Children, 22(1), 97-122. 
Arreman, I. E., \& Holm, A. (2011). Privatisation of public education? The emergence of independent upper secondary schools in Sweden. Journal of Education Policy, $26(2), 225$.

Bagga-Gupta, S. (2007). Aspects of diversity, inclusion and democracy within education and research. Scandinavian Journal of Educational Research, 51(1), 1-22.

Barker, G. P. (1996). 'Don't work too hard!' Really? Education Digest, 61(6), 17.

Barnes, M. (1999). Reform: Learning from Japan. Kappa Delta Pi Record, 35, 76-78.

Bereday, G. Z. F. (1980). The law and exclusion from schools in comparative perspective. Comparative Education Review, , 192-205.

Berhanu, G. (2008). Ethnic minority pupils in Swedish schools: Some trends in overrepresentation of minority pupils in special educational programmes. International Journal of Special Education, 23(3), 17-29.

Berhanu, G. (2010). Even in Sweden? Excluding the included: Some reflections on the consequences of new policies on educational processes and outcomes, and equity in education. International Journal of Special Education, 25(3), 148-159.

Berhanu, G. (2011). Inclusive education in Sweden: Responses, challenges and prospects. International Journal of Special Education, 26(2), 128-148.

Borovoy, A. (2008). Japan's hidden youths: Mainstreaming the emotionally distressed in Japan. Culture, Medicine and Psychiatry, 32(4), 552-576. 
Brodin, J., \& Lindstrand, P. (2007). Perspectives of a school for all. International Journal of Inclusive Education, 11(2), 133-145.

Brownell, M. T., Sindelar, P. T., Bishop, A. G., Langley, L. K., \& Seo, S. (2002). Special education teacher supply and teacher quality: The problems, the solutions. Focus on Exceptional Children, 35(2), 1.

Castricone, N. (1974). International comparative special education. Academic Therapy, $10(2), 239-243$.

Chambers, J. G., Parrish, T. B., \& Harr, J. J. (2002). What are we spending on special education services in the United States, 1999-2000? Report. special education expenditure project (SEEP).

Clark, M. J., \& Clark, K. (1970). Scandinavian programs for the mentally retarded: How they work. Chippewa Falls, WI: Northern Wisconsin Colony and Training School.

Crossley, M., \& Vulliamy, G. (1984). Case study research methods and comparative education. Comparative Education, 20(2), 193-207.

Danforth, S., \& Naraian, S. (2015). This new field of inclusive education: Beginning a dialogue on conceptual foundations. Intellectual \& Developmental Disabilities, 53(1), 70-85. doi:10.1352/1934-9556-53.1.70

Delpit, L. (2006). Other people's children: Cultural conflict in the classroom. New York: New Press. 
deVincentis, S. (1989). The function of culture in the development of social policy. Follow up to the seminar (Evanston, IL and Washington, DC, Spring 1988). Reston, VA, Evanston, IL: Council for Exceptional Children National Lekotek Center Swedish Information Center.

Ek, U., Westerlund, J., Holmberg, K., \& Fernell, E. (2011). Academic performance of adolescents with ADHD and other behavioural and learning problems -a populationbased longitudinal study. Acta Paediatrica, 100(3), 402-406.

Ellington, L. (2005a). Japanese education. Retrieved from http://iisdb.stanford.edu/docs/120/digest5.pdf

Ellington, L. (2005b). Japanese education. Stanford program on international and crosscultural education (SPICE). Retrieved from http://spice.fsi.stanford.edu/docs/japanese_education\#1

Elliott, J., \& Lukes, D. (2008). Epistemology as ethics in research and policy: The use of case studies. Journal of Philosophy of Education, 42(s1), 87-119.

EU Education, Audiovisual and Culture Executive Agency, Eurydice. (2011). National system overview on education systems in Europe: Sweden. September 2011. Retrieved from http://eacea.ec.europa.eu/education/eurydice/documents/eurybase/national_summary sheets/047_SE_EN.pdf 
Giota, J., Lundborg, O., \& Emanuelsson, I. (2009). Special education in comprehensive schools: Extent, forms and effects. Scandinavian Journal of Educational Research, 53(6), 557-578. doi:10.1080/00313830903302083

Goto, Y. (2008). Cultural commentary: Critical understanding of the special support education in social contexts. Disability Studies Quarterly, 28(3), 15-15.

Greene, J. P. (2007). Fixing special education. Peabody Journal of Education, 82(4), 703-723.

Grunewald, K. (1997). Effect of social and educational policies on the number of persons with mild mental retardation in Sweden. Mental Retardation, 35, 218-220.

Haynes, C. (2000). Teachers' skill ratings of children with learning disabilities: A comparison of the United States and Japan. Annals of Dyslexia, 50, 215-238.

Holler, R. A., \& Zirkel, P. A. (2008). Section 504 and public schools: A national survey concerning “Section 504-only” students. NASSP Bulletin, 92(1), 19-43.

Holloway, S. D. (1988). Concepts of ability and effort in Japan and the United States. Review of Educational Research, 58(3), 327.

Huddart, H. (1981). In R. Jackson (Ed.), An analysis of vocational education for the handicapped in Sweden; Wessex studies in special education (pp. 75-100). Winchester, England: King Alfred's College. 
Isaksson, J., Lindqvist, R., \& Bergström, E. (2007). School problems or individual shortcomings? A study of individual educational plans in Sweden. European Journal of Special Needs Education, 22(1), 75-91. doi:10.1080/08856250601082323

Isaksson, J., Lindqvist, R., \& Bergstrom, E. (2010). 'Pupils with special educational needs': A study of the assessments and categorising processes regarding pupils' school difficulties in Sweden. International Journal of Inclusive Education, 14(2), 133-151. doi:10.1080/13603110802504176

Johannesson, I. A., Lindblad, S., \& Simola, H. (2002). An inevitable progress? Educational restructuring in Finland, Iceland and Sweden at the turn of the millennium. Scandinavian Journal of Educational Research, 46(3), 325-339.

Kakizawa, T., Douglas, G., \& Kagawa, K. (2000). Students with visual impairments in special and mainstream schools in Japan: A survey. Journal of Visual Impairment \& Blindness, 94(4; 4), 218-228.

Kavale, K. A. (1988). Learning disability and cultural-economic disadvantage: The case for a relationship. Learning Disability Quarterly, 11(3), 195-210.

Kayama, M., \& Haight, W. (2012). Cultural sensitivity in the delivery of disability services to children: A case study of Japanese education and socialization. Children \& Youth Services Review, 34(1; 1), 266-275. 
Kayama, M. (2010). Parental experiences of children's disabilities and special education in the United States and Japan: Implications for school social work. Social Work, $55(2), 117-125$.

Kjellin, M. S., \& Wennerström, K. (2006). Classroom activities and engagement for children with reading and writing difficulties. European Journal of Special Needs Education, 21(2), 187-200.

Kjellin, M. S., \& Granlund, M. (2006). Children's engagement in different classroom activities. European Journal of Special Needs Education, 21(3), 285-300.

Kjellin, M. S. (2007). Classroom niches for skilled readers and for children with reading and writing difficulties. Scandinavian Journal of Educational Research, 51(1), 6379.

Lincoln, Y. S., \& Guba, E. G. (1985). Naturalistic inquiry. Beverly Hills, Calif.: Sage Publications.

Lloyd, J. W., \& Lloyd, P. A. (2015). Reinforcing success. What special education could learn from its earlier accomplishments. Remedial and Special Education, 36(2), 7782.

Lundahl, L. (2005). A matter of self-governance and control--the reconstruction of Swedish education policy: 1980-2003. European Education, 37(1), 10-25.

Lundstrom, K. (1969). Special education in Sweden. The Volta Review, 528-538. 
Marshall, C., \& Rossman, G. B. (2006). Designing qualitative research (4th ed.). Thousands Oaks, Calif.: Sage Publications.

Masawa, G., Seo, M., \& Kusanagi, S. (1993). Teacher education to enhance the quality of special education in Japan. Peabody Journal of Education, 63, 44-59.

Mattson, E. H., \& Roll-Pettersson, L. (2007). Segregated groups or inclusive education? an interview study with students experiencing failure in reading and writing. Scandinavian Journal of Educational Research, 51(3), 239-252.

Meijer, C. J. W. (1998). Integration in Europe: Provision for pupils with special educational needs. Trends in 14 European countries. Middelfart, Denmark: European Agency for Development in Special Needs Education.

Ministry of Education, Culture, Sports, Science and Technology (MEXT). (2006). Basic Act on Education (provisional translation) Act No. 120 of December 22, 2006. Retrieved from http://www.mext.go.jp/english/lawandplan/1303462.htm

Ministry of Education, Culture, Sports, Science and Technology (MEXT). (2008). Basic plan for the promotion of education (provisional translation). Retrieved from http://www.mext.go.jp/english/lawandplan/1303463.htm

Ministry of Education, Culture, Sports, Science and Technology (MEXT). (N/A). Special needs education. Retrieved from http://www.mext.go.jp/english/elsec/1303763.htm

Miron, G. (1996). Free choice and vouchers transform schools. Educational Leadership International, , 77-80. 
Murakami, Y., \& Meyer, H. (2010a). Culture, institutions, and disability policy in Japan: The translation of culture into policy. Comparative Sociology, 9(2), 202.

Murakami, Y., \& Meyer, H. (2010b). Culture, institutions, and disability policy in Japan: The translation of culture into policy. Comparative Sociology, 9(2), 202-221. doi:10.1163/156913310790788471

Nagano, M., \& Weinberg, L. A. (2012). The legal framework for inclusion of students with disabilities: A comparative analysis of Japan and the United States. International Journal of Special Education, 27(1; 1), 128-143.

Narita, S. (1992). Japanese special education today: Issues and implications. Tokosuka, Japan: National Institute of Special Education.

National Center for Education Statistics. (2015). Children and youth with disabilities. Retrieved from http://nces.ed.gov/programs/coe/indicator_cgg.asp

Noah, H. (1984). The use and abuse of comparative education. Comparative Education Review, 28(4), 153-165.

Organisation for Economic Cooperation and Development. (1983). The education of the handicapped adolescent: The transition from school to working life. Paris, France: OECD Centre for Educational Research and Innovation.

Organisation for Economic Cooperation and Development. (2012). Education at a glance: OECD indicators 2012. Sweden. Retrieved from 
http://www.oecd.org/sweden/EAG2012\%20-\%20Country\%20note\%20-

\%20Sweden5.pdf

Ortner, S. (1984). Theory in anthropology since the sixties. Comparative Studies in Society and History, 26(1), 126-126-166.

Peak, L. (1991). Learning to go to school in Japan: The transition from home to preschool life. Berkeley: University of California Press.

Persson, B. (1998). Who needs special education? International Journal of Educational Research, 29, 107-117.

Persson, B. (2008). On other people's terms: Schools' encounters with disabled students. European Journal of Special Needs Education, 23(4), 337-347.

Pouragheli, N. (2008). Privatizing Sweden's education. Retrieved from $\underline{\text { http://politics.lilithezine.com/Privatizing-Swedens-Education.html }}$

Provasnik, S., Gonzales, P., \& Miller, D. (2009). US performance across international assessments of student achievement: Special supplement to the condition of education 2009. NCES 2009-083. National Center for Education Statistics,

Reagan, T. (2005). Non-western educational traditions: Indigenous approaches to education thought and practice.

Riessman, C. K. (1993). Narrative analysis. Newbury Park, CA: Sage Publications. 
Roll-Pettersson, L., \& Mattson, E. H. (2007). Perspectives of mothers of children with dyslectic difficulties concerning their encounters with school: A Swedish example. European Journal of Special Needs Education, 22(4), 409-423.

Rydberg, E., Gellerstedt, L. C., \& Danermark, B. (2009). Toward an equal level of educational attainment between deaf and hearing people in Sweden? Journal of Deaf Studies \& Deaf Education, 14(3), 312-323. doi:10.1093/deafed/enp001

Rydberg, E., Gellerstedt, L. C., \& Danermark, B. (2010). The position of the deaf in the Swedish labor market. American Annals of the Deaf, 155(1), 68-77.

Samuels, C. (2011). Special education court cases on the rise. Retrieved from http://blogs.edweek.org/eweej/speced/2011/01/special_education_court_decisi.html

Schifter, L. (2011). High school graduation of students with disabilities: How long does it take? Exceptional Children, 77(4), 409-422.

Schleicher, A. (2014). PISA 2012: Results in focus. What 15-year-olds know and what they can do with what they know OECD.

Sheridan, E. M. (1993). Reading disabilities: Are there fewer in Japan? Reading Horizons, 33(3), 245-257.

Skiba, R. J., Simmons, A. B., Ritter, S., Gibb, A. C., Rausch, M. K., Cuadrado, J., \& Chung, C. (2008). Achieving equity in special education: History, status, and current challenges. Exceptional Children, 74(3), 264-288. 
Stenhouse, L. (1979). Case study in comparative education: Particularity and generalisation 1. Comparative Education, 15(1), 5-10.

Suzuki, M. (2008). Review of Shinohara, M. (1986) Ideological critiques of the mainstream special education discourse on "protecting the educational rights of children with disabilities": Focusing on human reciprocity, or ensuring development? Disability Studies Quarterly, 28(3)

Sweden. SE. (2012). Education in Sweden: Lessons for life. Retrieved from http://www.sweden.se/eng/Home/Education/Basic-education/Facts/Education-in$\underline{\text { Sweden/ }}$

The Swedish National Agency for Education (Skolverket). (2011). An overview of the Swedish education system. Retrieved from http://www.skolverket.se/om$\underline{\text { skolverket/andra-sprak-och-lattlast/in-english/the-swedish-education-system }}$

Tsumori, M. (1998). Education and care for children with special needs. International Journal of Early Childhood, 33(1), 79-82.

Tyack, D., \& Cuban, L. (1995). Tinkering toward utopia: A century of public school reform. Cambridge: Harvard University Press.

U.S. Department of Education. (2010). Thirty-five years of progress in educating children with disabilities through IDEA. Retrieved from https://www2ed.gov/offices/list/osers/idea35/history/idea-35-history,pdf 
United Nations Development Programme. (2013). Total public expenditure (current and capital) on education expressed as a percentage of GDP. Retrieved from http://hdr.undp.org/en/content/expenditure-education-public-gdp

Wehman, P., Sima, A. P., Ketchum, J., West, M. D., Chan, F., \& Luecking, R. (2015). Predictors of successful transition from school to employment for youth with disabilities. Journal of Occupational Rehabilitation, 25(2), 323-334.

Wessman, L. (1970). In I. Ekstrom (Ed.), Organization of special education in Sweden; proceedings of the first international seminar of special education and rehabilitation of the mentally retarded (pp. 130-138). Malmo, Sweden: International Association for the Scientific Study of Mental Deficiency.

Wong, M. E. (2004). Higher education or vocational training in "riryoka"? post-school choices of visually-impaired students. part 2, Japan. British Journal of Visual Impairment, 22(2), 59-65.

Woodward, J., \& Ono, Y. (2004). Mathematics and academic diversity in Japan. Journal of Learning Disabilities, 37, 74.

World Population Statistics. (2013). Japan population 2013. Retrieved from http://www.worldpopulationstatistics.com/japan-population-2013/

Zhang, D., Katsiyannis, A., Ju, S., \& Roberts, E. (2014). Minority representation in special education: 5-year trends. Journal of Child and Family Studies, 23(1), 118127. 
Zilversmit, A. (1993). Changing schools: Progressive education theory and practice, 1930-1960. Chicago: University of Chicago Press. 